NBSIR 84-2813

Center for Materials Science

\title{
Polymer \\ Science and \\ Standards \\ Division
}

\section{Annual Report 1983}

\author{
U.S. DEPARTMENT \\ OF COMMERCE \\ Naitonal Bureau \\ of Standards
}


POLYMER SCIENCE AND STANDARDS DIVISION

Annual Report 1983

U. S. Department of Commerce National Bureau of Standards Polyners B1dg. (224), A305 Washington, DC 20234 (301) $921-3734$ 



\section{Polymer Science \\ and Standards \\ Division}

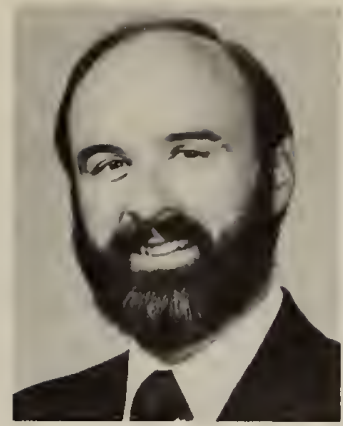

LESLIE E. SMITH, Acting Chief. Dr. Smith was born in New York. He earned degrees in chemistry from Case Institute of Technology and Catholic University. Except for a three year period of graduate study, he has been with NBS since 1964.

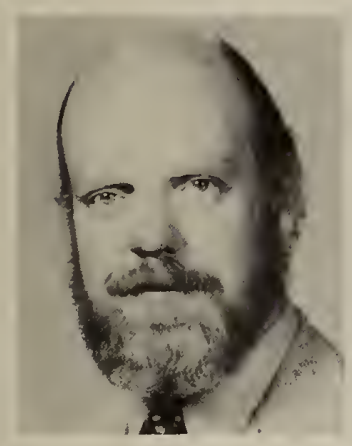

MARTIN G. BROADHURST, Acting Deputy Chief. Dr. Broadhurst was born in Washington, D.C., and earned degrees in physics at Western Maryland College and Pennsylvania State University. After teaching at Pennsylvania State, he joined NBS in 1960.
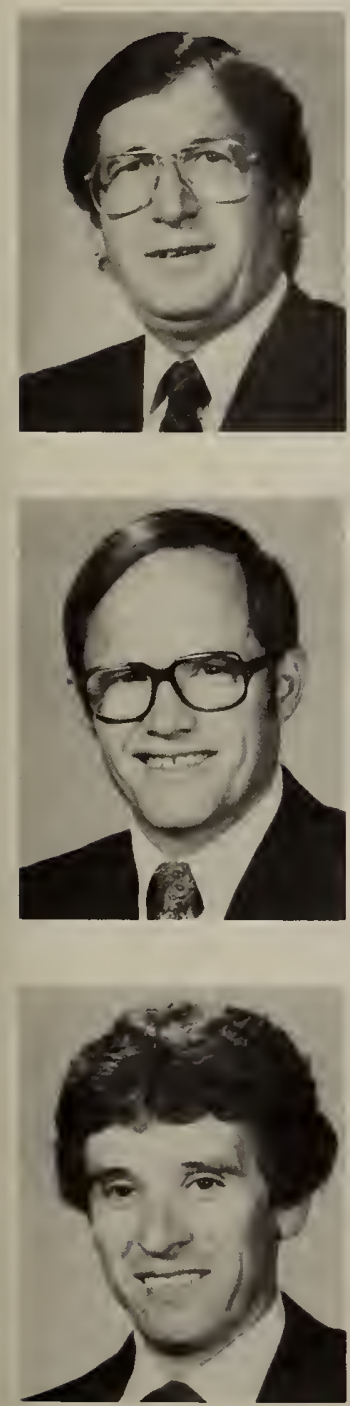

G. THOMAS DAVIS, Acting Group Leader, Electrical Properties. Dr. Davis was born in Montour Falls, New York. He earned degrees in chemical engineering at Cornell University and physical chemistry at Princeton University. Prior to joining NBS in 1964, he worked for Esso Research and Engineering Co. and taught at the University of Virginia.

BRUNO M. FANCONI, Group Leader, Composites and Mechanical Performance. Dr. Fanconi was born in Merced, California. He earned degrees in physical chemistry from the University of California at Los Angeles and the University of Washington. He joined the staff of NBS in 1971.

ISAAC C. SANCHEZ, Group Leader, Polymer Blends. Dr. Sanchez was born in San Antonio, Texas. He earned degrees in physical chemistry at St. Marys University (Texas) and at the University of Delaware. Prior to joining the NBS staff in I977, he worked at the Xerox Research Laboratories and was on the Polymer Science and Engineering faculty at the University of Massachusetts.

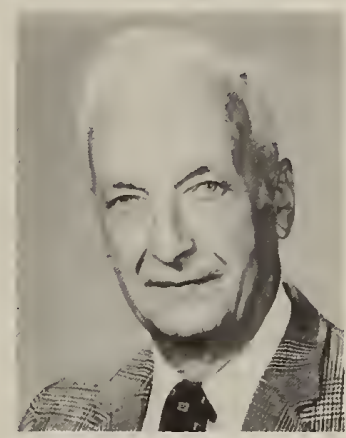

ANTON PETERLIN, Assistant Chief. Dr. Peterlin was born in Yugoslavia. He earned the M.S. in mathematics at the University of Ljubljana and Ph.D. in physics at the University of Berlin. From 1939 to 1960 he was professor of physics in Ljubljana and from 1949 to 1959 was head of the Josef Stefan Institute. Before joining NBS in 1973, he was director of the Camille Dreyfus Laboratory.

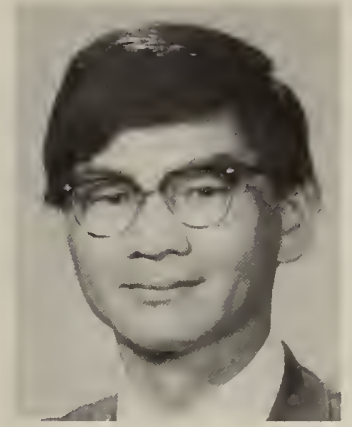

FRANCIS W. WANG, Acting Group Leader, Chemical Performance and Standards. Dr. Wang was born in Peikang, Taiwan. He earned the B.S. and M.S. in chemical engineering at California Institute of Technology and the Ph.D. in physical chemistry at the University of California, in San Diego. He joined the staff of NBS in I972 after a postdoctoral period at the Polytechnic Institute of New York.

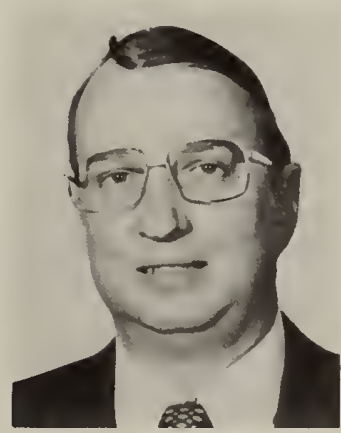

JOHN A. TESK, Acting Group Leader, Dental and Medical Materials. Dr. Tesk was born in Chicago, Illinois. He holds the degrees of B.S., M.S. in Metallurgy and Ph.D. in Materials Science from Northwestern University. Prior to joining NBS in 1978, he was at the University of Illinois, Chicago, on the staff at Argonne National Laboratory, Director of R\&D with the Dental Division of Howmedica, Inc. and Director of Education Services at IGT. 


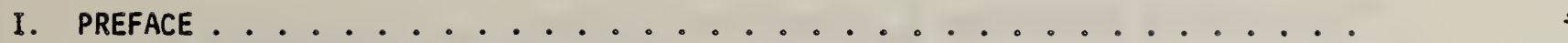

II. POLYMER THEORY FOR STRUCTURE AND PROCESSING . . . . . . . . . . . . . 1

- Classification of Structure Inducing Transitions in Polymers

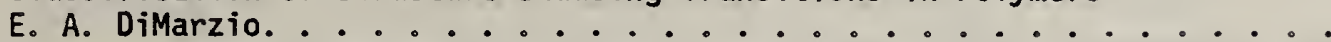

- Monte Carlo Modeling of the Kinetics of Polymer Crystal Growth

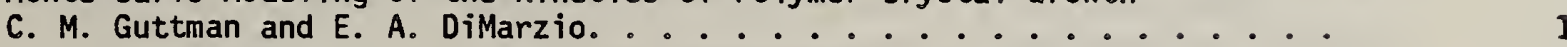

- Block Copolymers with Lamellar Morphology: Chain Dimensions in Directions Parallel and Perpendicular to the Lamellae

E. A. DiMarzio.....................

- Translational Diffusion Constant of Linear and Branched Chains

C. M. Guttman ...................

- Processes Involving Conformational Changes in Polymers

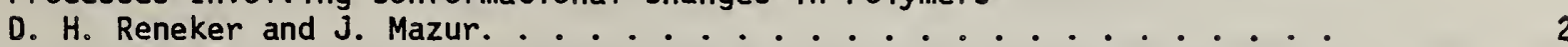

III. POLYMERS STANDARDS FOR PROCESS CONTROL AND EQUITY . . . . . . . . . . . 4

- Self-Calibrating Gel Permeation Chromatography; P. H. Verdier . . . . .

- Critical Evaluation of Mark-Houwink Relation for Linear Polyethylene;

H. L. Wagner ..................

- Characterization of UItrahigh Molecular Weight Polyethylene; H. L. Wagner and J.G. Dillon. ....................

- Characterization of Polyisobutyl Methacrylate; H. L. Wagner . . . . . 6

- Dynamics of Polymer Chains; P. H. Verdier and D. E. Kranbueh1 ...... 6

- Automated Liquid Size Exclusion Chromatography; J. D. Barnes and

F. L. McCrackin . . . . . . . . . . . . . . . .

- Span of an Adsorbed Polymer Chain in a Lattice Random Walk Model;

R. J. Rubin .....................

- Monte Carlo Calculations of the Hydrodynamic Radii of Polymers;

F. L. MeCrackin and C. M. Guttman ................

- Standards for Gas Transmission Measurements; J. D. Barnes . . . . . . . 8

- ASTM Activities; J. D. Barnes . . . . . . . . . . . . . 9

- Random-Walk Model of Chain Polymer Adsorption. Behavior at Critical Energy and Relation to Reflecting Boundary Condition; R. J. Rubin . . . . . .

- Expansion Factor of a Pärt of Polymer Chain in Good Solvent Measured by Small Angle Neutron Scattering; C. C. Man, T. P. Lodge, E. J. Amis,

Y. Matsushita ...................

- Temperature Dependence of Dynamic Light Scattering in the Intermediate Momentum Transfer Region; T. P Lodge, C. C. Han, A. Z. Akcasu . . . . . .

- Dynamic Scattering from Bimodal Pclymer Solutions; B. Hammouda, A. Z. Akcasu, T. P. Lodge, C. C. Han . . . . . . . . . . . . . . . . .

- Oynamic Light Scattering Measurements of Polystryrene in Semidilute Solutions E. J. Amis, C. C. Han and Y. Matsushita. 
IV. BLENDS PROCESSING . . . . . . . . . . . . . . . . . . . . . . 11

- Forced Rayleigh Scattering; E. J. Amis and C. C. Han . . . . . . . . . 11

- SANS and SAXS Studies on Molecular Conformation of a Block Copolymer in Microdomain Space; H. Hasegawa, T. Hashimoto, H. Kawai, C. C. Han,

T. P. Lodge, A. J. Amis . . . . . . . . . . . . . . .

- Novel Fluorescence Method for Polymer Blend Phase Diagrams; F. W. Wang and

R. E. Lowry .......................

- Nonosecond Fluorimeter for Measurements of Polymer Phase Diagrams; F. W. Wang, F. L. MeCrackin, J. D. Barnes, and W. H. Grant. . . . . . . . . 12

- Installation of a Laboratory for Polymer Synthesis; B. J. Bauer . . . . . 12

- Digital Small Angle Scattering Camera; J. D. Barnes . . . . . . . . 13

- New Correlation Discovered for Surface Tension of Simple and Polymieric

Liquids; I. C. Sanchez. ..................

V. TESTS, STANDARDS AND CHARACTERIZATION FOR MANUFACTURING AND DURABILITY OF DENTAL

AND MEDICAL MATERIALS. . . . . . . . . . . . . . . . . .

- Dental Composite and Sealant Chemistry and Related Developments;

J. M. Antonucci, G. M. Brauer, R. L. Bowen, J. W. Stansbury,

R. E. Deht, and N. W. Rupp ..................

- Dental Adhesion Research; R. L. Bowen, G. M. Brauer, J. M. Antonucci,

J. W. Stansbury, D. W. Misra, E. Cobb, and J. M. Cassel. . . . . . .

- Dental Cements; G. M. Brauer, J. M. Antonucci, and J. W. Stansbury . . .

- Wear Mechanisms and Durability of Dental Composite Materials. Effect of Degree of Cure; W. L. Wu, J. E. McKinney, E. E. Debelius, and J. M. Cassel ......................

- Porcelain Fused-to-Metals and Dental Ceramics; J. A. Tesk, W. deRijk, H. R. Kase, G. Widera, D. L. Menis, and R. W. Hinman .........

- Dental Casting Alloys and Investments; J. A. Tesk, R. W. Waterstrat,

S. Hirano, R. W. Hinman, H. R. Kase, W. deRijk, and H. Argentar. . . . .

- Dental Chemistry, Crystallography, and Preventive Treatments; W. E. Brown,

L. C. Chow, M. Mathew, S. Takagi, M. Tung, G. L. Vogel, T. M. Gregory,

C. M. Carey, B. Sieck, and C. Schreiber. . . . . . . ......

- Clinical and Laboratory Investigation of Dental Amalgams, Composites and Glass Ionomer Cements; N. W. Rupp, G. C. Paffenbarger, and

R. W. Waterstrat ....................

- Characterization of Porosity in Porous Polymeric Implant Materials;

R. E. Dehl .......................

- Surgicar Bone Cement Modification; G. M. Brauer, J. A. Tesk, J. W. Stansbury, W. L. Wu, and J. E. McKinney . . . . . . . . . . . . . . .

- Dental and Medical Materials Standards; J. M. Cassel, J. A. Tesk, G. M. Brauer, N. W. Rupp, and W. Derijk. 
VI. MECHANICAL DURABILITY OF POLYMERS ...................

- Molecular Processes Associated with Mechanical Deformation and Failure; B. M. Fanconi, D. L. VanderHart, S. J. Kryder, J. R. Havens,

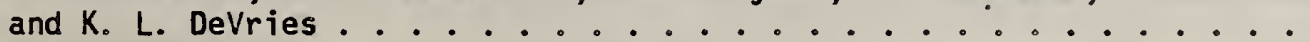

- The Morphology of Crystalline Polymers; F. A. Khoury; L. H. Bolz, and J. Mazur. ....................

- The Necking of Semicrystalline Polymers in Tension; L. J. Zapas and J. M. Crissman. .....................

- A Study of the Morphology and Mechanical Properties, and Their Dependence on Thermal History and Processing of Ultra High Molecular Weight Polyethylene; J. M. Crissman ....................

- Information Handbook on Polymeric Materials Used in Orthopedic Devices; J. M. Crissman and G. B. McKenna .................

- Instabilities in the Flow of Viscoelastic Fluids; R. W. Penn and L. J. Zapas. .........................

- The Distribution of Stresses in Crazes at the Tips of Uniformly Extending

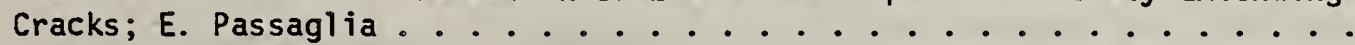

- Physical Ageing in Polymeric Glasses Subjected to Large Deformations; G. B. McKenna and A. J. Kovacs ................

- The Effect of Elastic and Plastic Deformation on the Solubility of Ethyl Acetate Vapor in Polyvinylidene Fluoride $\left(P V F_{2}\right)$; J. C. Phillips and A. Peterlin.

VII. PROCESSING AND RELIABILITY OF POLYMER COMPOSITES. . . . . . . . . . . .

- Interlaminar Fracture of Composites; D. L. Hunston, G. W. Bullman, and

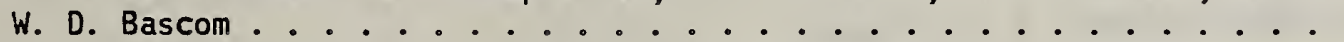

- Failure Behavior of Particle Reinforced Polymer Composites; D. L. Hunston, A. J. Kinloch, and S. S. Wang. ...............

- Cure Monitoring in Polymers and Composites via Nondestructive Techniques; D. L. Hunston, B. M. Fanconi, F. I. Mopsik, C. C. Yu, and H. Weisshaus . .

- Chemorheology in Polymerization Systems Initiated by Oxygen; D. L. Hunston and G. W. Bullman. . . . . . . . . . . . . . . . .

- Novel Excimer Fluorescence Method for In-Situ Monitoring of Composite Materials Manufacturing; F. W. Wang, R. E. Lowry, and W. H. Grant. . . .

- Analysis of Thermoset Resin Polymerization Kinetics; G. A. Senich, R. E. Florin, and J. C. Phillips................

- Measurement of Photopolymerization of Polymers by Thermal Methods;

J. H. Flynn. . . . . . . . . . . . . . . . . . .

VIII. ADDITIVE MIGRATION AND CHEMICAL DURABILITY OF POLYMERS. . . . . . . . .

- Prediction of the Long Term Stability of Polyester-Based Recording Media; D. W. Brown, R. E. Lowry, and L. E. Smith. . . . . . . . . . . . 
- Hydrolytic Degradation of Polyester Polyurethanes; D, W. Brown, R. E. Lowry, and L. E. Smith.

- Studies With Polyether Polyurethane Tubing; D. W. Brown, R. E. Lowry, and L. E. Smith.

- Solar Type Photolytic and Thermal Degradation of Plates of Poly(Methyl) Methacrylate; B. Dickens...................

- Evaluation of IC-Encapsulants; S. S. Chang and J. R. Maurey.

- Variable Heating Rate Thermal Analysis; J. H. Flynn.

- Measurement of Additive Diffusion by Fluorimetry; F. W. Wang, B. F. Howell, and F. L. McCrackin.

- Migration of Low Molecular Weight Materials in Polymers Under Simultaneous Swelling Conditions; C. M. Guttman and S. S. Chang ...........

- Migration of Styrene Monomer From Polystyrene; S. S. Chang and J. R. Maurey

- Chromotographic Studies of Migration From Polymers; G. A. Senich. . . . .

- Solubilities and Partition Coefficients of BHT in Polyolefins and Various Solvents; S. S. Change and J. R. Maurey ...............

- Determination of Segmental Mobility by Picosecond Fluorescence Spectroscopy;

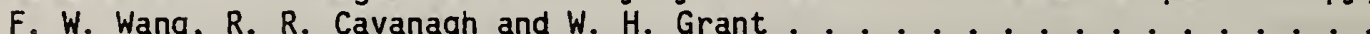

IV. MICROSTRUCTURE AND PERFORMANCE OF DIELECTRIC PLASTICS . . . . . . . . . . . . . 48

- Automated Dielectric Measurements; F. I. Mopsik . . . . . . . . . . 48

- Dielectric Properties of Polyvinylidene Fluoride; A. J. Bur

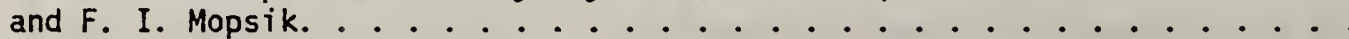

- Dielectric Loss Spectra of Polymers in the Microwave Region; A. J. Bur . . .

- Conducting Polymers; C. K. Chiang. . . . . . . . . . . . . .

- Fast Ion Transport in Solid Polymers; C. K. Chiang, G. T. Davis, C. A. Harding, and T. Takahashi ....................

- Structure and Performance of Piezoelectric Polymers; G. T. Davis and M. G. Broadhurst . . . . . . . . . . . . . . . . . 52

- Polarization and Space Charge Studies; A. S. DeReggi and F. I. Mopsik. . . . 52

- A Dynamic Polymer Pressure Transducer With Temperature Compensation; A. J. Bur and S. C. Roth . . . . . . . . . . . . . . . . .

- Acoustic Sensors; A. S. DeReggi. . . . . . . . . . . . .

- Spontaneous Polarization in Ferroelectric Polymers; A. S. DeReggi, S. C. Roth, G. T. Davis, and M. G. Broadhurst. ................

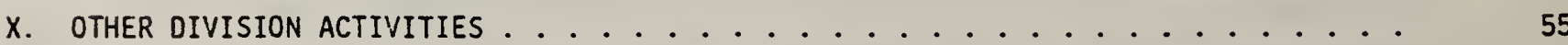

- Invited Talks. . . . . . . . . . . . . . . . . . . 55

- Seminars for Staff and Guests. . . . . . . . . . . . . . . 73 
- Technical and Professional Committee Participation and Leadership. . . . . 80

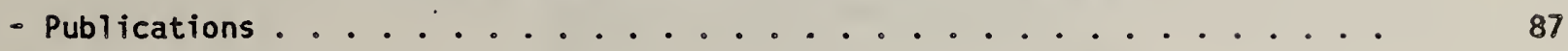

- Speciar Reports. . . . . . . . . . . . . . . . . . 95

-Books. . . . . . . . . . . . . . . . . . . . . 95

- Consulting and Advisory Services .................. 95

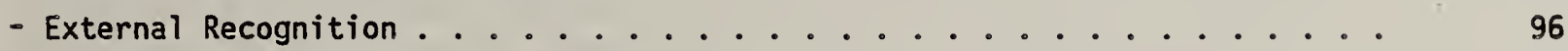

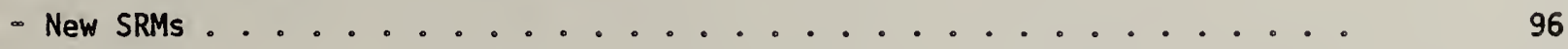

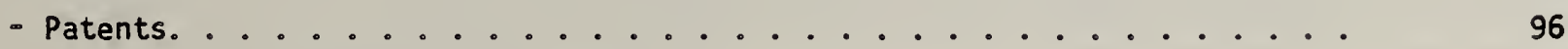

- Industrial Interactions. . . . . . . . . . . . . . . . 96

- Formal visits........................ . . . . . . 97 

Although synthetic polymers have been used as materials for technology during only three quarters of a century, they have left little of our economy, technology, industry, science, and culture untouched. We have moved rapidly into an age in which an evergrowing number of humanity's needs are served by polymers. The volume currently produced exceeds that of steel and forms the basis of industries which add over $\$ 106$ billion of value by manufacturer (a measure of the relative economic importance of manufacturing among industries) and provides 3.3 million jobs. Recent summaries show that polymers and polymer composites research already accounts for about 47 percent of the total industrial R\&D expenditure for metals, polymers, and inorganic materials. Among these materials, polymers also constitute about 40 percent of the value added by manufacturer, 45 percent of the jobs, 49 percent of the number of scientific publications, and 42 percent of the American Society for Testing and Materials (ASTM) standards.

Polymers have been shaped by intellectually sophisticated research which has been recognized by a number of Nobel Prizes. The resulting materials have contributed to national industrial productivity through their economy, ease of fabrication, and useful properties such as low density, corrosion resistance, and toughness. Polymers are energy efficient; the largest volume polymer, polyethylene, uses 29 percent of the energy per unit volume required by steel for conversion from raw to refined material and yields further savings in processing and shipping. In fact, studies have shown that for every barrel of oil used to make plastic products, approximately one barrel is saved in comparison with the use of the next best alternative material. These characteristics have enabled polymers to contribute to increased national economic strength both by creating entirely new products and by substituting for other materials in existing products.

Polymers have entered a widening range of increasing sophisticated and demanding applications. Polyvinylidene fluoride films are used as piezoelectric transducers in high fidelity sound systems, biomedical imaging devices and infrared sensors. Structural parts cf aircraft are constructed from composites with a polymer matrix and sometimes a polymer reinforcement. Polyurethane elastomers are used to pump blood in circulatory assist devices. The list of such examples is very long, and includes trends for the increased use of polymers in packaging, construction, transportation, national security, electronics, information handling, clothing, electrical appliances, energy, dentistry and medicine. Since polymer science and technology is on the steep part of the learning curve, these trends create many new needs for scientific concepts, measurements, standards, and data that can be used to ensure their reliable performance in the use, processing, and other phases of the material cycle. The programs of the Division are aimed at the fundamental scientific basis of these needs.

To identify the key needs with the best prospects for payoff, the managers of the Polymer Science and Standards Division make structured visits to industrial and Government laboratories. Visited sites include Bell Laboratories, E. I. DuPont de Nemours, Inc., 3M, Army R\&D Command, General Motors Corporation, Martin Marietta, and Lawrence Livermore Laboratories. Surveys have been conducted in the areas of durability, composites, and processing. Research Associates, working on joint NBS-industrial projects in polymers were sponsored by ten organizations during the past year including General Electiric Research Laboratories, Exxon Chemical Co., Martin Marietta, Arco Chemical Co., and the American Dental Association. These and other contacts with research associates, experts in industry, trade associations, standards associations, guest workers, Government, and academia ensure that the Division's efforts are directed toward fostering the effective use of polymers in solving generic national problems. These include the growth of the economy, national security, efficient Government, materials utilization, and conservation of critical materials.

Current activities are associated into areas concerned with phase behavior and characterization methods for polymer blends, processing and reliability of composites, long-term research on the scientific basis for mechanical reliability in load-bearing applications, chemical durability, lifetime prediction, migration of protective additives, characterization methods, standards for molecular composition and performance properties, dielectric properties, and reliable dental and medical materials. Substantial cooperative efforts are conducted with research associates from a number of industries, universities, and Department of Defense, the National Institutes of Health, the American Dental Association (ADA), the Food and Drug Administration, and the Bureau of Engraving 
and Printing (BEP). All these efforts lead the staff of the Division into an examination of the scientific basis of those properties which are of concern to people who use, design, and produce useful polymer objects. This work helps society capture, in safe and satisfying ways, the benefits of the increasingly widespread application of polymers.

\section{FY83 SIGNIFICANT ACCOMPLISHMENTS}

\section{POLYMER STANDARDS FOR PROCESS CONTROL AND EQUITY}

- A high performance liquid size exclusion chromatograph laboratory has been established for the determination of the molecular weight and molecular weight distribution of polymer samples. The apparatus has been automated and may be operated at temperatures up to $150^{\circ} \mathrm{C}$. A microcomputer system for automated data collection and reduction has been in routine use during FY83. To date approximately 3,000 chromatograms have been collected and analyzed.

- A new Research Associate Program has been established with the ARCO Chemical Company. The objective of this program is to establish the feasibility of applying size exclusion chromatography to samples of ultra high molecular weight polyethylene and other high molecular weight polyolefins.

- A critical evaluation of the Mark-Houwink parameters used in determination of the molecular weight of linear polyethylene has been carried out. Literature values of these parameters for six polyethylene solvents, commoniy used for viscosity and size exclusion chromatography measurements, were critically evaluated. A report has been written giving the recommended values of the Mark-Houwink parameters.

- Measurements of the cooperative diffusion coefficient and center of mass translational diffusion coefficient as a function of concentration and molecular weight have been made by dynamic light scattering. The observed molecular weight and concentration dependences are not in full agreement with scaling law predictions.

- The anisotropic expansion of a polymer chain has been investigated by small angle neutron scattering. Deuteration of different portions of a polystyrene chain enables the determination of the expansion factor for these different portions.

\section{BLENDS PROCESSING}

- Construction and testing of a Forced Rayleigh Scattering instrument has been completed. The method permits rapid determination of the extremely low diffusion rates commoniy encountered in polymers. It will be particularly useful for studies of the kinetics of phase separation of polymer blends. This instrument uses two crossing coherent laser beams to generate interference patterns in a specimen by. exciting photochromic labels on polymers. When the laser is turned off, diffusional processes cause the interference pattern to decay. The diffraction of a second laser beam of different wave length is used to "read" the diffusion controlled dissipation of the interference pattern. The alternating line pattern looks like a holographic grating to the second laser. Since the spaçing of the grating is between 1 and $10 \mu \mathrm{m}$, diffusion coefficients as small as $10-10 \mathrm{~cm}^{2} / \mathrm{s}$ can be determined quickly by monitoring the intensity decay of the diffraction.

- A review paper entitled "Bulk and Interface Thermodynamics of Polymer Alloys" was published in the 1983 issue of Annuai Review of Materials Science.

- Fluorescence energy transfer has been used to investigate the phase behavior of ternary blends of PMMA, PEMA, and PVF ${ }_{2}$. In this method the constituent polymers are chemically labeled with a small number of "acceptor" and "donor" chromophores. The fluorescence intensity of the donors will be reduced by the acceptors whenever the donor and acceptor chromophores are separated by small distances as in a homogeneous mixture. Phase separation increases the average distance between donors and acceptors and, therefore, reduces the efficiency of the energy transfer. This method can also be used to monitor the phase separation process as a function of time. 
- A new two-dimensional $x$-ray detector is being installed for SAXS measurements which will facilitate the study of spinodal decomposition in blends.

- NBS and the Society of Plastics Engineers (SPE) have instituted a joint program to provide data on the thermodynamics and nonequilibrium phase behavior of polymer blends and related systems. NBS will be responsible for the overall guidance of the technical aspects of the program with the aim of assuring reliability of the data evaluations and will provide a center for coordination with other data compilation centers.

- A new correlation has been discovered for the surface tension of simple and polymeric liquids [ $\mathrm{J}$. Chem. Phys., 79: 405; 1983]. This correlation relates the surface tension of a liquid to its isothermal compressibility and mass density. It has been shown to be applicable to quantum liquids, hydrogen bonding liquids, and polymeric liquids.

\section{TESTS, STANDARDS, AND CHARACTERIZATION FOR MANUFACTURING AND DURABILITY OF DENTAL AND MEDICAL MATERIALS}

- Clinical observations in outside laboratories combined with our clinical and laboratory results revealed a potential short term laboratory observation which may reduce the need for long term clinical studies to evaluate the durability of new alloy systems. Dimensional changes of restricted and unrestricted amaigam specimens correlated directly with the clinical observations of durability using three widely accepted but markedly different alloys. If this correlation continues to be consistent for other alloys, 4 to 8 month in vitro data can be used to predict 3 year clinical data.

- The characterization of porosity in two porous polymeric implant materials is being continued, at the request of the Office of Medical Devices (FDA). A method of preparing histograms of mercury porosimetry data has been devised, leading to the conclusion that the distribution of pore radii in the materials is log-normal. The pore volume percentage and pore size (mean intercept length) have been measured for both materials by the method of quantitative microscopy. This work is important to establish reliable and accurate methods for characterizing the porosity of implant materials.

- Polymeric resins in commercial dental composites cured at the in situ temperature $37{ }^{\circ} \mathrm{C}$ have been found to be undercured, and are thus susceptable to polymeric softening by certain solvents present in the oral environment. Increasing the degree of cure by increasing the cure temperature has been found to increase the in vitro wear resistance of commercial composites presoaked in certain food simulating chemical liquids. Although this method of increasing the degree of cure may be impractical for in situ restorations, the wear results imply the incorporation of more flexible chain polymers and a mismatch between solubility parameter values of resin and common oral solvents to achieve more durable composites.

- Most of the modern dental alloys are based on nickel. Since there is some concern over latent carcinogenicity and allergic sensitization from nickel alloys, the use of titanium as a potential substitute is promising. A major impediment to the widespread use of titanium alloys is their poor castability under dental laboratory conditions. A recent accomplishment of NBS is the production of a successful titanium alloy casting using a magnesium oxide investment.

- Dental cements with superior physical properties have been prepared by incorporating cement and composite components into a new hybrid material. The lack of polymerization inhibition by the recently developed hexyl vanillate-EBA-ZnO cements allows the addition of monomers and reinforcing fillers to the cement formulation. The extremely high strength, low solubility, and excellent adhesion of the resulting cements indicate their application as durable, intermediate restoratives.

- A tooth structure conserving procedure for bonding composite restorative materials to dentin has been developed which utilizes brief applications of a cleanser mordant aqueous solution of ferric oxalate, a surface active comonomer and couping agent. The difficulties in bonding to dentin have been serious obstacles to the successful placement of many restorations. Tensile tests on restorations using this technique revealed frequent cohesive failure in the composite and occasionally, in the dentin. 
- The chemical resistance and overall durability of the polymeric matrices currently used in dental composite restorative materials is a significant factor limiting their service life. The use of low surface energy polymeric binders based on the highly fluorinated oligomeric multifunctional methacrylate, PFMA, is a feasible method of enhancing the hydrophobicity, oleophobicity, stain resistance and chemical stability of dental composites. A variety of hydrocarbon and fluorocarbon diluent comonomers have been used with PFMA to yield composites with good mechanical properties, reduced microleakage, and extremely low water sorption.

- A new technique for determining the water sorption of composites and sealants by nearinfrared spectroscopy was developed and appears to be a more sensitive method for measuring water uptake than the standard gravimetric procedure. In contrast to the latter method which requires immersion of relatively large specimens in water, long equilibration times, and the measurement of small changes in large numbers, the near-IR method has the following advantages: (1) thin specimens $(<100 \mu \mathrm{m})$ which have short equilibrium times are used, (2) comparative measurements after immersion in water versus simple exposure to atmospheres of $100 \%$ relative humidity can be made, and ( 3 ) the time dependent water sorption behavior of the specimen may be easily monitored.

- In addition to their utility in dental composite materials, we have found that polythiols such as pentaerythritol tetra(3-mercaptoproprionate), PETMP, reduce the setting times of denture base materials while improving the esthetics and color stability of the cured product. When used in modest quantities in liquid component (e.g. methyl methacrylate plus amine accelerator) of a commercial surgical bone cement formulation, it was shown to be effective in reducing the peak temperature of the exotherm without adversely affecting dough and setting time or the compressive strength of the cured cement. A more moderate peak temperature is desirable in surgical bone cement since it reduces the chance of inflammatory or other adverse responses in contiguous tissues.

- Conventional polycarboxylate cements are based on the acid-base reaction of a poly(alkenoic acid), e.g. poly(acry! ic acid), and a basic powder (e.g. Zno). Ionically crosslinked polymeric matrices are formed that are rigid and hydrophilic. We have explored the feasibility of obtaining less rigid and hydrophobic dental cements by the reaction of liquid dimer (DA) and trimer (TA) acids with a variety of basic powders (e.g. $\mathrm{ZnO}, \mathrm{MgO}, \mathrm{CaO}$, etc.). Many of these cements have shown excellent resistance to brittle failure and very low solubility both in water and lactic acid solutions. The versatile nature of cements obtained from $D A$ and $T A$ with different base components suggests many potential dental applications.

- Dental cement formulations in which syringate esters have partially or completely replaced the structurally similar hexyl vanillate ingredient have been evaluated. These new cements maintain the highly desirable physical properties obtained with the vanillate-EBA-Zno cements. Since syringic acid has been shown to significantly inhibit caries formation, the syringate cements might also be caries-reducing.

o A simple technique for evaluating castability of dental alloys was developed at NBS. The castability value, $c_{v}$, of several alloys was determined. It was found that the same simple mathematical expression could be used to express $c_{v}$ as a function of alloy casting temperature and mode temperature for each alloys at a 99.5 percent confidence level.

- A relatively easy to use test specimen/methodology was developed for characterizing porcelain fused-to-metal (PFM) bond strength. It was found that a cumulative failure plot using Weibull distribution analysis shows clear distinctions between systems; such distinctions are not apparent from comparing the means of failure strengths in the different systems.

\section{MECHANICAL DURABILITY OF POLYMERS}

- Mechanical failure in fatigue of ultra high molecular weight polyethylene has been associated with a critical concentration of chain scissions. The number of chain scissions at failure has been found to be dependent on the initial molecular weight. Approximately twice as many chain scissions are required to produce failure in a polyethylene of twice the initial molecular weight. 
- The structure of ultrahigh modulus polyethylene is characterized by carbon-13 nuclear magnetic resonance. The profile of relaxation times indicates the presence of three components, and the intermediate component is identified with interfacial material. It can be concluded that significant contribution to the heat of melting arises from this region. Time constants for the long component vary significantly with sample preparation. An interpretation of their differences is that cold drawing produces crystallites which contain more crystal defects than in melt crystallization.

- A detailed investigation of inhomogeneous deformation has been completed on quenched samples of isotactic polypropylene under a wide variety of stress and strain histories in uniaxial extension. Using the data, a surface of isochrones of stress versus strain was constructed from which it was possible to predict the point at which inhomogeneous deformation will occur for other strain histories. It has been shown that the predicted behavior is consistent with a theory recently developed in our laboratory.

- The stress in the craze at the tip of a uniformly moving crack has been shown to be a function of the derivative of the displacement under the assumption that the craze thickens by a drawing process. The stress is thus identified as the drawing stress. The stress distributions have been obtained from theoretical analysis and show a peak at the craze tip and one at the crack tip, in agreement with experimental observations.

- The physical ageing behavior of polymethyl methacrylate glasses has been investigated as a preliminary to a larger program to measure dilatometrically the volume changes of polymer glasses subjected to increasing torsional deformation after various thermal treatments. The results revealed significant discrepancies as compared to previous studies and models of physical ageing in polymer glasses. In the linear viscoelastic range, the relaxation isotherms, measured at. increasing ageing times, cannot be superimposed by mere shifting along the time axis below some critical temperature range. Such effects may be accounted for by a chiange in the shape of the short time relaxation spectrum with increasing ageing time.

\section{PROCESSING AND RELIABILITY OF POLYMER COMPOSITES}

- The compressive strength of continuous fiber reinforced composites is sensitive to the delamination damage. There is a need to develop polymer matrix materials with improved resistance to both initial damage and subsequent growth of this damage into delamination. In addition, design data are needed to predict the growth rate of delamination. The effect of sample lay-up pattern on interlaminar fracture behavior has been studied and guidelines for specimen design have been formulated. Investigations of the temperature and rate dependence of interlaminar fracture of brittle and tougin polymer matrix materials as well as composites made from these materials revealed that the effects were negligible in the composites in contrast to the behavior for the resins alone.

- An extensive study was performed to examine the viscoelastic fracture behavior of a rubber-modified epoxy. Rubber modification is one way of improving the brittle nature of polymer matrix materials. Based on these studies, a mechanism for toughening has been formulated. The mechanism involves both dilatation via cavitation in the rubber particles or at the particle-matrix interface and shear plastic flow of the epoxy matrix.

- Time domain dielectric spectroscopy has been applied to the post curing of vinyl ester matrix materials. The measurements were carried out on the recently developed time domain dielectric spectrometer which has the capability to measure the dielectric properties of a material over a very wide range of time (or frequencies) in a single experiment. Preliminary studies have shown that it is possible to follow changes in molecular mobilities that would otherwise be very difficult to detect because they appear only in the very low frequency range. This type of information is particularly useful since the final degree of cure is often a critical parameter in processing.

- A novel excimer fluorescence technique has been developed for monitoring in situ and nondestructively the processing of components made of fiber-glass-reinforced composite materials. The method monitors the resin viscosity that must be carefully controlled during processing to avoid high rejection ratios of these components. 
- Rapidly curing thermoset matrix resins could greatly reduce processing time and permit a large increase in the volume of fabricated composite output. Radiation initiated polymerization is being examined as a method for obtaining rapid cure. An experimental program to measure the overall rate and determine reaction kinetics is in progress. The effects of initiator structure, concentration, and solubility, reactant concentration, fiber thickness, reaction temperature, and radiant intensity are being studied. This program is providing a better understanding of the chemistry that occurs during processing of thermoset polymers, and is aiding in the identification of materials for particular applications.

\section{ADDITIVE MIGRATION AND CHEMICAL DURABILITY OF POLYMERS}

- A method for predicting the useful lifetime of archival films made of poly(ethylene terephthalate) has been developed. The prediction was based on newly measured kinetic data on the hydrolytic degradation of this polymer. The results provide realistic estimates of when poly(ethylene terephthalate) archival films should be recopied.

- The relative contributions of thermal and photolytic degradation in a common solar glazing material poly(methyl methacrylate), PMMA, were elucidated. Sheets of PMMA were exposed to controlled environments and the molecular weight was measured as a function of depth. The results show the importance of oxygen in reducing molecular weight, and of unpolymerized raw material in converting the initial products of degradation into a form more sensitive to oxygen attack.

- A theoretical model has been developed to explain the complicated but important case of migration of an additive from a polymer into a solvent that swells the polymer. The model relates the non-fickian migrant loss behavior to the non-Fickian sorption behavior. The predictions of the model for the very short time and the very long time agree with the limiting behavior seen in experiments.

- Extraction experiments and concentration profile experiments have been carried out using fluorimetry to determine the diffusion coefficients of additives in low-density polyethylene. An experimental value for the diffusion coefficient of 3-tert-buty-4hydroxyanisole (BHA) in polyethylene at $30^{\circ} \mathrm{C}$, in the absence of a solvent, has been determined.

- Picosecond fluorescence spectroscopy has been used to determine the segmental mobility of methacrylate polymers and pyrenelabeled alkanes. This measurement is one way of determining the kinetics of resin curing in polymer composites.

\section{MICROSTRUCTURE AND PERFORMANCE OF OIELECTRIC PLASTICS}

- The time domain dielectric spectrometer recently developed at NBS is being transferred to the user community. Through the Research Associate program, the General Electric Co. is reproducing the instrument for their laboratory and the IMass Corp. will produce the instrument for sale. The main features of the instrument have been described in a manuscript submitted for publication in the Review of Scientific Instruments.

- The speed and accuracy of dielectric measurements afforded by the time domain dielectric spectrometer have been used to study the influence of crystal phase and orientation on the dielectric properties of polyvinylidene fluoride. These relaxations are expected to provide information useful in predicting the performance and durability of the polymer as a piezoelectric and pyroelectric transducer. The spectrometer has also been used to follow changes which occur during post-cure of epoxy resin composites and to differentiate between morphologies which exist in some polymer blends.

- Copolymers of vinylidene fluoride and trifluoroethylene containing 52 to 78 mole percent VDF have been examined as ferroelectrics. The ferroelectric to paraelectric transition temperature increases with VDF content and involves a crystal-crystal transition which is similar for all compositions. Favorable piezoelectric response can be obtained even without mechanical orientation and polarization reversal occurs over a narrow range of electric field near $400 \mathrm{kV} / \mathrm{cm}$. This work was done cooperatively with Bell Laboratories. 
- The thermal pulse technique for measuring space charge and polarization distribution has been successfully applied to films of polyvinylidene fluoride, fluorinated ethylene propylene copolymer, and nylon-11 in cooperative work with $3 \mathrm{M}$, Bell Laboratories, and Naval Ocean Systems Command.

- Piezoelectric polymer sensors which compensate for the pyroelectric response arising from adiabatic heating of the polymer or its surroundings are being developed under a contract with the Air Force. The Building Research Division will evaluate the gauges as pressure transducers in a variety of soil environments as a part of the contract.

- A Research Associate agreement between NBS and Alta Technology Inc. has been reached. The work will focus on the performance of piezoelectric polymer arrays with special emphasis on minimizing cross-talk between elements. The polymer properties required for non-propagating acoustic modes will be specified and experimental arrays will be developed and evaluated.

- Rigorous upper and lower bounds for the dielectric constant of a semicrystalline polymer containing anisotropic crystals has been calculated. The model has been applied to polyvinlyidene fluoride and found to be in good accord with data resulting from different processing such as orienting and poling.

- Ionic conductivity in polyethyleneoxide to which $\mathrm{NaI}$ has been added has been found to exhibit a maximum at a salt concentration far below that where a crystalline complex between the polymer and salt first appears. Conduction in non-crystalline regions rather than specific pathways within the crystal as frequently proposed has important consequences in guiding further research on ion transport in polymers.

In the following pages, the activities of the Division are presented formally in eight tasks, each described separately:

POLYMER THEORY FOR STRUCTURE AND PROCESSING

POLYMER STANDARDS FOR PROCESS CONTROL AND EQUITY

BLENDS PROCESSING

TESTS, STANDARDS, AND CHARACTERIZATION FOR MANUFACTURING AND DURABILITY OF DENTAL AND MEDICAL MATERIALS

MECHANICAL DURABILITY OF POLYMERS

PROCESSING AND RELIABILITY OF POLYMER COMPOSITES

ADDITIVE MIGRATION AND CHEMICAL DURABILITY OF POLYMERS

MICROSTRUCTURE AND PERFORMANCE OF DIELECTRIC PLASTICS 



\section{POLYMER THEORY FOR STRUCTURE AND PROCESSING}

Processing routes, particularly those involving phase transitions, can produce a wide range of microstructures which must be controlled to optimize desirable properties. An improved scientific basis for characterization of molecular aspects of polymer molecules and their conformational behavior will provide industry with tools to control processing and for nondestructive evaluation. Better specifications for polymers in industrial and defense applications will also result.

Basic features of phase transitions in polymers are modeled in ways that incorporate the kinetics of the transition. Included are phase transitions relevant to polymer blends, composites, and glasses. Both transitions that occur in single molecules and those that occur in collections of molecules are addressed.

The usefulness of gel permeation chromatography and quasielastic light scattering for molecular characterization and processing is enhanced by work describing the relation between branching of polymer molecules and these measures of molecular size.

The mechanical behavior at long times of classes of high volume polymers, particularly in the fiber form, were modeled. The conformational and vibrational behavior of defects which rotate and translate the polymer chain were calculated.

\section{Classification of Structure Inducing Transitions in Polymers}

\section{E. A. Di Marzio}

Phase transitions in polymers constitute a rich subject area. There are more transitions occurring in polymer systems than in any other subject area. This is because the nature of the fundamental unit, which is the molecule, can be infinitely varied in its complexity (chain length being only one degree of complexity). The following transitions and phases occurring in polymers have been treated by the author: crystals, liquid crystals, liquids, glass transition, Helix-Random Coil transition, collapse transition, the transition associated with adsorption onto a surface, and finally polymerization which can be viewed as a transition. These various transitions can be made to couple to one another giving a hierarchy of structures involving feedback and control. An attempt is being made to describe each of the transitions with a simple mathematical treatment that captures the physical basis of the transition. This will facilitate treatments which couple the various transitions to one another.

Phase transitions are useful to processing science because a slight change in one of the control variables (examples: temperature, pressure, affinity, shear rate) can result in a sudden appearance of structure. The profusion of structure inducing phase transitions in polymer systems warrants study.

\section{Monte Carlo Modeling of the Kinetics of Polymer Crystal Growth}

\section{M. Guttman and E. A. Di Marzio}

A Monte Carlo simulation of polymer crystal growth from the melt was completed this year. This two-dimensional model approximates growth of a polymer crystal using the model of laying down full stems one at a time. The model allows us to explore the growth of polymer crystals over a wide range of temperatures. More particularly the model extends theoretical studies into the region of temperature most used in polymer processing. The simulation was developed to obtain quantitative estimates of the accuracy of previous approximate mathematical approaches. We were able to show that analytical expressions previously developed for growth at small undercoolings were very accurate. However, in the high undercooling regions--those of most interest from the point of view of processing--we showed the analytical expressions were not at all accurate. Furthermore, previcus analytical developments made no predictions on the effects of detailed geometric structures on the surface. We showed that the traditional site on site model does not produce space filling polymer crystals at high undercooling. A hexagonal lattice model of the crystal yields space filling crystals but does not show the rapid upsweep observed experimentally at high undercoolings. Only if we allow growth on a hexagonal lattice and growth on more than one growth plane does the Monte Carlo model mimic the experimental data well.

The requirements of this Monte Carlo model are consistent with earlier modeling the small angle neutron scattering (SANS) on highly undercooled polymer crystals where we found it necessary to invoke a two-growth plane model to explain the SANS data. 


\section{E. A. Di Marzio}

The packing entropy (arising from competition for space) is dependent on the orientation of molecules. In block copolymers the chains are stretched out in the direction perpendicular to the planes of the lamellae. The packing entropy contributes a term that results in a shrinking of the transverse dimensions. Quantitative estimates are made. The result is a substantial improvement over existing treatments which predict a negligible shrinking in the transverse direction. The experimental data seem to show a shrinkage even larger than what we predict. The effect on dissolved homopolymers is described. Block copolymers (chemical blends) can be used to stabilize polymer blends.

\section{Translational Diffusion Constant of Linear and Branched Chains}

\section{M. Guttman}

With the advent of experimental equipment in which both static and quasi-elastic light scattering may be done on the same sample, the ability to obtain both a radius of gyration and a hydrodynamic radius on the same system is possible. For branched chains this offers the hope of obtaining structural information about branching heretofore unavailable. Since the character and details of the branching of a polymer molecule determine its processability as well as its ultimate physical properties and mechanical strength, such information on branching is invaluable.

One theory currently available to relate these two quantities to the polymer structure is the Kirkwood Riseman theory of the translational diffusion constant in solution. However there has been found to be a discrepancy between the experimental value of the translational diffusion coefficient of polymer molecules, both branched and unbranched, in dilute solution and that obtained from Kirkwood Riseman theory using an analytical Gaussian model of the chain. The discrepancy is 15 percent for linear chains and nearly 50 percent for the ideal star branched chains studied.

Current work at this laboratory approaches this problem in two ways. In one, the hydrodynamic radius is computed using the Kirkwood Riseman formulation for both linear and branched chains created by a computer simulation in which both excluded volume and interaction energies are included. These calculations have shown that about half of the $15 \%$ deviation found between theory and experiment previously was attributable to these corrections for linear chains. Currently work on branched chains is in progress.

In a second approach analytical modeling was done on chains in which local chain structure but not excluded volume is taken into account. Earlier work showed that the analytical model currently available which is used to compare theory and experiment shows a very slow approach to its large molecular weight, $M$, limit. It is only in this limit that the Gaussian theory and experiment can be meaningfully compared. Thus corrections to the large $M$ values used to compare with experiments were not possible even for polymers of high molecular weight." In this effort analytical modeling of chains with local structure and stiffness was done and the first two corrections to the large $M$ limit of the hydrodynamic radius of the chains were computed exactly. It was shown that the effect of chain structure on each of these three terms was different. Thus the simple ideas of statistical length used earlier were found to need improvement.

\section{Processes Involving Conformational Changes in Polymers}

\section{H. Reneker and J. Mazur}

Polyethylene fibers offer the possibility of very high modulus and strength since the backbone bonds are very strong and the cross sectional area of the molecule is small. Achievement of these exceptional properties requires alignment of the molecules, so they must move past each other and eliminate unfavorable molecule conformations during processing. To maintain the high strength, the molecules must not slide along their axes after the fiber is formed. In this work molecular scale processes which are involved in the formation and mechanical deformation of fibers are modeled as a basis for processing methods to produce strong, stable polymer fibers. 
The molecular vibrations associated with crystallographic defects in polyethylene called dispirations, disclinations, dislocations, and twist boundaries were modeled. These defects provide mechanisms for translating the polymer chain along its axis or rotating it. Characteristic vibrations associated with the defects may make it possible to observe the presence and behavior of defects by examining the vibrational spectrum of polyethylene.

It was found that the above crystallographic defects have a lowest longitudinal frequency in the range of 85 to $105 \mathrm{~cm}^{1}$ associated with motions of the ends of the defect region parallel to the chain axis. The ends may simultaneously twist or move sideways, but the longitudinal motion is dominant. This defect vibration may couple to any of a large number of normal vibrational modes of the polyethylene crystal. This coupling tends to split the lowest longitudinal frequency of the defect into a band of frequencies. The longitudinal vibration of the defect has a reasonably high predicted Raman intensity, so probably contributes to the Raman spectrum either as a broad band or an increase in the background intensity in the range of 85 to $105 \mathrm{~cm}{ }^{1}$. The defect regions have other characteristic vibrational modes at lower frequencies. These modes have displacements which are transverse to the chain axis, both twist and shear. The low frequency transverse modes do not contribute to the Raman spectrum, but some may be infrared active. It appears that vibrational spectroscopy can provide information needed to characterize crystallographic defects in polyethylene.

The transport of polymer molecules in the melt, which is fundamentally involved in the processing of polymers, proceeds by the motion of segments of a molecule. This gives rise to a relatively rapid process which produces local rearrangements and a slower process which ultimately moves the center of mass of the molecule over macroscopic distances. In cooperation with scientists in the Polymer Science and Standards Division, and the Jozef Stefan Institute in Ljubljana, the diffusion of polymer molecules in the melt is observed using magnetic resonance measurements of spin echo in a magnetic field gradient. The interactions of adjacent molecules seem to play an unexpectediy important role in controlling the time evolution of the molecular transport process. This was observed by measuring the transport in mixtures of polyethylene and paraffin. The data indicate that velocity correlations persist for surprisingly long times and that unusually long times are required for the magnetic dipolar interactions of the protons on the molecule to average to zero. These experiments promise new and fundamental insight into the kinematics of entangled melts of flexible polymer molecules. 
This task provides standard reference materials (SRMs) to the $\$ 30$ B/year synthetic polymers producing industry. Molecular weight SRMs are used extensively in industry to calibrate gel permeation chromatographs (GPCs) which are used routinely to measure polymer molecular weights and molecular weight distributions. The latter are the most important physical properties in determining a polymer's processability and its ultimate performance. Gas transmission and rubber SRMs are used in process control applications.

Improved characterization techniques are essential to providing improved SRMs. A fundamental knowledge of polymer solution properties such as osmotic pressure, viscosity, diffusional behavior, and scattering power is essential to this goal. As a consequence, a majority of the activities in this task involve experimental and theoretical studies of polymer solution properties. As a spinoff of our characterization activities, this task also provides data and concepts which are useful in polymer processing.

\section{Self-Calibrating Gel Permeation Chromatography}

\section{P. H. Verdier}

Gel permeation chromatography (GPC) is a widely accepted technique for estimating the molecular weight distribution (MWD) of high polymers. However, the usefulness of the conventional GPC apparatus is limited by the need to provide calibrants for each polymer measured of known molecular weight over the entire molecular weight range in which the MWD is significantly different from zero. The calibration depends, among other things, upon the chemical nature, degree of branching, etc., of the polymeric material so that each new material requires a fresh calibration. The so-called "universal calibration" hypothesis, while useful, is limited to comparisons of polymers of similar shape and, in any event, is inadequate for quantitative determinations. Some instruments, one of which is commercially available, attempt to circumvent the need for calibrants by adding a single-angle light scattering detector to the usual concentration-sensitive detector. However, this does not allow the extrapolation to zero scattering angle which is required, in principle, to relate scattering intensity to molecular weight. In addition, qualitative information on the variation of scattering with angle, normally required to give assurance that meaningful results are being obtained, is not available.

A light-scattering detector for the GPC has been designed and built which measures, in real time, scattered intensity as a function of scattering angle. The apparatus is controlled by a dedicated minicomputer in a way that allows scattering to be measured as a function of both scattering angle and concentration. Continuous monitoring of the variation of scattering with angle while the chromatogram is being obtained allows immediate identification of difficulties such as association, microgel formation, etc., which would affect the validity of the molecular weights obtained, an important consideration for work on new and unistudied materials. In addition to molecular weight, the mean-square radius (radius of gyration) can be obtained as a function of molecular weight, at least in the higher ranges of molecular weight, providing useful information for the characterization of branched polymers. The second virial coefficient, a measure of polymer-polymer interactions of both current and continuing interest to solution-properties theorists, can also be obtained as a function of molecular weight.

During the current year, a series of tests of the instrument has been carried out. Excessive quantities of stray light were found at very small scattering angles, due apparently to the inevitable mismatch in index of refraction between the solvent employed (tetrahydrofuran) and the entrance window of the scattering cell. While these effects can be calibrated out, they materially reduce the accuracy of the final results. A new scattering cell has therefore been designed, with cell geometry which minimizes detection of entrance-window scattering and with improved internal blackening and decreased cell volume. Improved controls for alignment of the scattering cell have also been designed. Fabrication of the new components is expected to be completed by the end of FY83.

\section{Critical Evaluation of Mark-Houwink Relation for Linear Polyethylene}

\section{H. L. Wagner}

One of the most important physical properties of a polymer is its average molecular weight which largely determines its processability and its performance properties. Since absolute 
determinations of molecular weight are time consuming and are not made routinely, relative methods, such as solution viscosity or size exclusion chromatography (GPC), are used instead.

The limiting viscosity number (LVN) or the intrinsic viscosity--and the molecular weight, $M$, are related by a simple power law:

$$
\operatorname{LVN}=[\eta]=\mathrm{KM}^{\mathrm{a}}
$$

where $K$ and a are empirical constants which depend on the specific solvent and the temperature. An incomplete compilation of these data can be found in handbooks, but a critical assessment is not available. Yet these data are used extensively to obtain molecular weights as well as to calibrate size exclusion chromatography columns.

Detailed examination of the original literature revealed that many of the viscosity determinations used to obtain $K$ and a were made with whole polymers rather than fractions so that the values of these parameters depend on the molecular weight distribution. In other cases the absolute molecular weights of the fractions were determined indirectly from viscosity measurements in a solvent with known values of $K$ and $a$. By measuring the viscosity of these same fractions in a second solvent, values of $K$ and a for this solvent may now be determined using the molecular weights measured in the first solvent. Such an indirect determination using the molecular weights measured in the first solvent. Such an indirect determination adds to be uncertainty. Determinations such as these were rejected in favor of direct determinations of molecular weight by absolute methods such as light scattering and osmotic pressure for narrow molecular weight distribution samples.

After reviewing and evaluating all the papers dealing with a particular polyethylene solvent, the best Mark-Houwink parameters for that solvent were chosen. This was done for each of six solvents commonly used for viscosity and size exclusion chromatography measurements. These solvents are: decalin, 1,2,4 trichlorobenzene, 1-chloronaphthalene, tetralin, o-dichlorobenzene, and xylene. In addition, the $K$ values for several theta solvents $(a=0.5)$ were compiled. A report has been written giving the results of this evaluation and the recommended values of the Mark-Houwink parameters.

\section{Characterization of Ultrahigh Molecular Weight Polyethylene}

\section{H. L. Wagner and J. G. Dillon}

Ultrahigh molecular weight polyethylene (UHMWPE), one of the most extensively employed surgical implant materials, is usually characterized for specification and control by limiting viscosity number (intrinsic viscosity) rather than by a direct measurement of molecular weight such as light scattering. The viscosity may be measured at zero shear rate in the low shear viscometer developed in this laboratory, or by a relatively simple measurement in a high shear rate capillary viscometer, which must then be empirically corrected for shear rate. For many polymers an empirical Mark-Houwink relation is available for relating the molecular weight to the limiting viscosity number. Such a relation exists for ordinary commercial polyethylene, but not for UHMWPE, al though it is often assumed the same relation holds. To determine the Mark-Houwink parameters for these higher molecular weights, fractions of varying molecular weights are required as well as a method for measuring their molecular weights.

Two fractionations were carried out using the stir crystallization technique to give 17 fractions with zero shear rate viscosities ranging from $9 \mathrm{dL} / \mathrm{g}$ to $50 \mathrm{dL} / \mathrm{g}$. A special procedure was necessary to dissolve some of the higher molecular weight fractions. Using the uncertain Mark-Houwink equation derived from data for lower molecular weight fractions, an integral distribution curve was obtained for the whole polymer. Although this results in some uncertainty in the absolute values of molecular weight, the general features of the curve are of interest. The molecular weight of the bulk of the material lies between 700,000 and 10 million. There is a suggestion of a bimodal distribution peaking at 1.1 million and 4.5 million. A report in the literature indicates that Ziegler-Natta type polyethylenes, such as this sample, have very narrow distributions and in the same paper some possible reasons for this are put forth. This does not seem to be true of this sample which appears to be broad, possibly because it may be a blend of two polymers, as indicated by the bimodal distribution curve.

Liquid size exclusion chromatography (GPC), is a very convenient, indirect, method of determining molecular weight and molecular weight distribution of polymers with molecular weight 
species of no greater than several hundred thousand. Recently, column manufacturers have developed new columns which they claimed would surpass this limit. To determine whether one such column set could separate UHMWPE, a very dilute solution was injected at the highest possible temperature, $148{ }^{\circ} \mathrm{C}$. Although instrument problems were encountered, it was nevertheless evident that a large fraction of the polymer eluted at the exclusion limit and was not separated. Because the exclusion limit is about 1 million for polyethylene, it was concluded that the whole polymer contains a substantial amount of material greater than this molecular weight. Since it is also possible that some degradation occurred, no determinations of the lower molecular weight components were made. A sample of one of the lower molecular weight fractions with a limiting viscosity number of 11.5 was also run, and although no instrument problems were encountered, it was evident that a substantial portion of the fraction was also eluted beyond the exclusion limit. Since a molecular weight distribution could not be obtained for even this relatively low fraction, no further attempt was made to inject any other higher molecular weight fractions.

Pennings has examined by electron microscopy the structures formed during hydrodynamic crystallization, the fractionation process used here, and found a "shish-ke-bab" structure. He believes the fibrils are the high molecular weight constituents crystallizing first and the overgrowths are the lower molecular weight species which crystallize subsequently.

Since the fractions cannot be characterized by size exclusion chromatography, with the columns now available it will be necessary to measure their molecular weight by light scattering. A low angle laser light scattering apparatus will shortly be available for this purpose.

\section{Characterization of Polyisobutyl Methacrylate}

\section{H. L. Wagner}

The Chemical Systems Laboratory at Aberdeen Proving Grounds has asked the National Bureau of Standards (NBS) to consolidate and evaluate the data on the characterization of polyisobutyl methacrylate carried out by two commercial laboratories. A review of the data indicated that interlaboratory agreement was poor and that in the development of a polymer or process it would be best for control purposes to have the characterization performed in a single laboratory. The reproducibility of limiting viscosity numbers (intrinsic viscosities) was much poorer than usual in the one laboratory in which it was carried out.

Some experiments were also conducted by NBS on the dependence of viscosity on shear rate of a sample of polyisobutyl methacrylate with a molecular weight of about 1.5 million. This was found to be negligible in the solvent tributyl phosphate.

\section{Dynamics of Polymer Chains}

\section{P. H. Verdier and D. E. Kranbueh1}

The dynamic behavior of polymer chains is important to improving both characterization and processibility of polymers. The principal methods used to estimate molecular weight, and in particular gel permeation chromatography, the only method available at present for estimating distribution in molecular weight, are dynamical, nonequilibrium experiments which depend in part upon the relaxation of polymer chains in dilute solution. In its present state, the theory of these systems does not allow the calculation of molecular weight directly from measured quantities; a series of calibrants of known molecular weight is required. Improvements in the theory could reduce or even remove the need for calibrants, allowing the determination of molecular weight distribution of polymers for which calibrants are not available.

The processibility and the final characteristics of both plastics and elastomers depend in large part upon the relaxation behavior of the high-polymer chains of which these materials are primarily composed. More realistic theoretical treatments of polymer melts and elastomers, which must start with more realistic treatment of the dynamical behavior of the individual chains, will lead to more efficient processing and better fabricated end products. A major shortcoming of the present theory is its inability to treat chain entanglements in a realistic way. Present work is aimed primarily at the study of entanglement effects, using a combination of analytical and computer simulation techniques. The simulation results provide data on simple model systems and serve to guide the development of better theoretical treatments. 
Work in this area is proceeding along two main lines. First, as a step in the direction of investigating melt properties, simulation studies are being carried out of systems of many interacting chains, with chain densities spanning the range from dilute solutions to concentrated solutions and melts. An initial set of studies of these systems has been completed and the results analyzed. As the concentration increases from the dilute-solution limit, entanglement effects cause the chain relaxation times to increase dramatically. The results are consistent with the wellestablished free volume theory, with a slight modification to take account of chain connectivity. These results will be published during the coming year. Second, a series of studies is under way to assess the magnitude of artifacts due to the simple move rules employed and to constraining the chains to lie on space-fixed lattices. Perhaps surprisingly, removing the requirement that the chains lie on lattices appears to increase the effects of excluded volume and entanglement upon chain relaxation times.

\section{Automated Liquid Size Exclusion Chromatography}

\section{J. D. Barnes and F. L. McCrackin}

A microcomputer system for automated data collection and reduction has been in routine use during FY83. To date approximately 3,000 chromatograms have been collected and analyzed. The studies that have used the system most extensively have been concerned with monitoring the degradation of poly(methyl methacrylate) as subjected to various photolytic and thermal oxidative stresses for research programs in the Center for Building Technology and the Center for Fire Research.

Areas requiring further effort include the generation of calibration curves defining the functional dependence of hydrodynamic volume on elution volume, the characterization of the background which must be subtracted from the chromatograms, and data management techniques for handling the large quantities of raw data and analytical results that are obtained. Enhancements to the software that will provide for easier user interaction with the graphically displayed data are currently being designed.

Techniques for dealing with data off line have been developed in order to allow users to analyze their data without interfering with the data collection process.

\section{Span of an Adsorbed Polymer Chain in a Lattice Random Walk Model}

\section{R. J. Rubin}

Recently [1] the probability density of the span, or extent, of an N-step adsorbed polymer chain in the direction normal to the solution surface was derived in deGennes' model [2] for weakly adsorbed chains. The corresponding probability of the span has now been derived in a discrete lattice random walk model of an adsorbed polymer chain [3]; and a paper reporting results is in preparation. It is noteworthy that in both models the following two measures of thickness of an adsorbed polymer chain have a different $\mathrm{N}$-dependence. The average span is proportional to $\mathrm{lnN}$ and the scale length of the exponential step density distribution [3] is independent of $N$. Although the foregoing results are derived in models which ignore the effect of excluded volume, we conclude that the average span increases at least as rapidly as $1 \mathrm{nN}$ in models where self-intersections are excluded.

[I] R. J. Rubin and G. H. Weiss, J. Chem. Phys. 78, 2039 (1983).

[2] P. G. de Gennes, Rep. Prog. Phys. 32, 187 (1969).

[3] R. J. Rubin, J. Chem. Phys. 43, 2392 (1965).

Monte Carlo Calculations of the Hydrodynamic Radii of Polymers

\section{F. L. McCrackin and C. M. Guttman}

The inverse radius $(1 / R)$ of single polymer chains on a cubic lattice and on a face centered lattice were computed. These chains were created by a Monte Carlo simulation in which both excluded volume and energetics of interaction were taken into account. Values of $I / R$ are calculated for various values of the energy of interaction. Thus the effect of going from good solvent to a theta solvent is modeled. The hydrodynamic radius of the polymer chain is computed from the values of $1 / R$ using Kirkwood-Riseman theory and compared to experimentally obtained hydrodynamic radii. The 
hydrodynamic radii obtained from the Monte Carlo simulation are shown to be in better agreement with the experimental data obtained at the theta point then those hydrodynamic radii computed for Gaussian coils.

The $1 / R$ and the radius of gyration obtained from the Monte Carlo calculations are fit as a function of both $N$ and temperature to a modification of the deGennes blob model of the polymer chain. The fit is found to be reasonably good.

Analytical calculations of $1 / R$ as a function of the chain length $N$ for chains in which the local structure of the chain is taken into account but in which no excluded volume has been included have been developed. This was done to see if the better agreement of the Monte Carlo is the result of some details of local structure which are in the Monte Carlo model but not in the Gaussian coil models. Analytical models developed so far suggest that the deviation between the Monte Carlo and the analytical models at the theta point is not a result of such local structure but rather a result of the effect of the excluded volume.

The inverse radii of two interacting chains have been computed and are being interpreted as the properties of polymers in dilute solution.

\section{Standards for Gas Transmission Measurements}

\section{J. D. Barnes}

Gas transport properties of polymeric materials are the major determinant of suitability for end use in a great variety of applications ranging from food and beverage packaging, industrial gas separation processes, and sterilization of medical devices, to substrates and packages for semiconductor products. Gas molecules are useful as probes of materials properties at the microstructural leve1. The measurement system for gas transport properties has suffered from a lack of reliable standards.

NBS is addressing these needs by developing improved measurement technology and by characterizing polymeric materials for use as Standard Reference Materials (SRMs). The revised certificate for SRM 1470 has been released for transmission to users of the material.

During the recertification process for SRM 1470 it was observed that a mathematical model based on the permeation of a gas obeying ideal Fickian diffusion failed to properly predict the transition to steady-state permeation behavior for $\mathrm{CO}_{2}$ in SRM 1470. Erforts to construct appropriate numerical solutions to the transport equation for systems exhibiting partial immobilization in their gas transport properties has been initiated in an attempt to provide a better fit to the experimental data. It is expected that this activity will lead to a better understanding of transport processes in cases where the diffusing species appear to exhibit a weak binding to polymer matrix.

The users of SRM 707, Standard Reference Material for Water Vapor Transmission Rate, have been forced to consider needs for new standards because it was not possible to replace the SRM when supplies became exhausted. The originai (1972) certification of the SRM was based on measurements using ASTM E96, a cup test. Improved methods for measuring water vapor transmission in thin films have been developed since 1972 .

The material used in SRM 707 was similar to SRM 1470, and it has also become clear that this material is sensitive to water vapor and thus may not be an appropriate choice for a water vapor transmission SRM.

Dr. J. D. Barnes has been working with representatives of the Office of Standard Reference Materials and ASTM Committees F2, D20, and C16 to identify the state of the measurement art in the area of water vapor transmission in plastic films, to identify the needs for new standards, and to identify materials that might be suitable for SRMs.

The efforts involve: (1) an interlaboratory test program for which NBS is devising the protocol and analyzing the results, (2) identification of test methodology that is suitable for characterizing the water vapor transmission properties of materials to the necessary precision and accuracy, and (3) screening measurements on candidate materials to evaluate their suitability as SRMs. 


\title{
J. D. Barnes
}

J. D. Barnes serves as Chairman of ASTM Subcommittees F-2.93 and D-20.13. These subcommittees provide assistance in matters relating to the design of experiments for evaluating the precision of test methods for the parent ASTM Committees. This assistance takes the form of approval of round-robin protocols, critique of precision statements, and statistical education for task group leaders. Current projects include an interlaboratory test program for ter Vapor Transmission meäsurements, an interlaboratory test program for evaluating oxygen diffusion into package issemblies, and defining the proper form for formulating the standard deviation in ASTM test methods.

Computer programs for analyzing the results of interlaboratory test programs have been developed and are currently being field-tested by several task groups in Committee D-20. We expect to document these programs for general distribution so that ASTM task groups will have suitable tools for carrying out analyses of interlaboratory test programs using ASTM E-691, Standard Practice for Conducting and Analyzing Interlaboratory Test Programs for Studying the Precision of Test Methods.

Random-Walk Model of Chain Polymer Adsorption. Behavior at Critical Energy and Relation to Reflecting Boundary Condition

\section{R. J. Rubin}

It is found that there are subtle differences between the set of energyweighted random walks generated in the random-walk model of polymer chain adsorption at the critical energy and the corresponding set of random walks generated in the presence of a reflecting boundary. These subtle differences have their analog in the lattice model of self-avoiding random walks at the theta-point where volume exclusion of monomers is exactly compensated by monomer-monomer attraction in the limit of large $N$.

\section{Expansion Factor of a Part of Polymer Chain in Good Solvent Measured by Small Angle Neutron Scattering}

C. C. Han, T. P. Lodge ${ }^{1}$, E. J. Amis ${ }^{2}$, Y. Matsushita ${ }^{3}$

${ }^{1}$ Assistant Professor, University of Minnesota

2 Research Associate, University of Wisconsin

${ }^{3}$ Assistant Professor, Nagoya University

The radii of gyration of deuterium labeled parts in polystyrenes with narrow molecular weight distributions in good solvent (carbon disulfide) were determined by small angle neutron scattering (SANS): The expansion factors of the labelled parts were calculated from the observed radii of gyration and their unperturbed ones which were estimated from the unperturbed radius of gyration-molecular weight relationship of polystyrene in literature. The expansion factors of the labelled parts were smaller than those of the whole chains, but were larger than that of a whole chain with the same molecular weight as that part. This result is reasonable compared with a Monte Carlo calculation in literature and also with the perturbation theory.

\section{Temperature Dependence of Dynamic Light Scattering in the Intermediate Momentum Transfer Region}

\author{
T. P. Lodge ${ }^{1}$, C. C. Han, A. Z. Akcasu ${ }^{2}$ \\ ${ }^{1}$ Assistant Professor, University of Minnesota \\ 2 Professor, University of Michigan
}

Dynamic light scattering measurements have been made on dilute solutions of very high molecular weight polystyrenes in cyclohexane at $35.4^{\circ} \mathrm{C}, 45.0^{\circ} \mathrm{C}$, and $55.2^{\circ} \mathrm{C}$. The data have been analyzed in terms of the first cumulant $\Omega(q)$ of the dynamic structure factor $S(q, t)$, with particular emphasis on the intermediate momentum transfer region $\left(R^{-1} \ll q \ll \ell_{1}^{1}\right)$, for which theoretical predictions yield $\Omega(q) \sim q^{3}$. The temperature dependence of the magnitude off $\Omega(q)$ is examined in terms of a 
"modified blob" model for the equilibrium distribution function and the preaveraged Oseen tensor description of hydrodynamic interaction. The temperature dependence of the experimental results is found to be in very good agreement with the theoretical calculations, although the magnitude of $\Omega(q)$ at each temperature falis consistently slightly below ( $\sim 15$ percent) the theory, as reported previously for both good solvent and theta conditions.

\section{Dynamic Scattering from Bimodal Polymer Solutions}

B. Hammouda ${ }^{1}$, A. Z. Akcasu ${ }^{2}$, T. P. Lodge ${ }^{3}$, C. C. Han

1 Research Associate, University of Michigan

2Professor, University of Michigan

${ }^{3}$ Assistant Professor, University of Minnesota

Dynamic scattering matrix $S(q, t)$ for scattering from multimodal systems is formulated and explicit results in the case of bimodal systems are presented in the small-q limit. The total dynamic scattering function is expressed, in this limit, as a weighted sum of two exponentials with decay rates $\Gamma_{1}$ and $\Gamma_{2}$. Both the decay rates and the weighting factors are calculated in terms of the concentrations and molecular weights of the two components. The dependence of the apparent diffusion coefficient $D_{a p p}$ on concentrations of both components are calculated and the results are compared to the experimental data for polystyrene $\left(M_{w}=1.79 \times 10^{5}\right.$ and $\left.1.05 \times 10^{6}\right)$ in cyclohexane at the theta point. The magnitude of the interference effect on the concentration dependence of $D_{\text {app }}$ is studied quantitatively. The concentration dependence of the apparent diffusion coefficient and the collective diffusion coefficient in a single component system is expressed in terms of the pair correlation function for polymer molecules.

\section{Dynamic Light Scattering Measurements of Polystyrene in Semidilute}

\section{Solutions}

E. J. Amis ${ }^{1}$, C. C. Han and Y. Matsushita ${ }^{2}$

1 Research Associate, University of Wisconsin

${ }^{2}$ Assistant Professor, Nagoya University

Measurements of the cooperative diffusion coefficient $D_{c}$ and a center of mass transiational diffusion coefficient, $D_{s}$ have been made by dynamic light scattering for the polystyrene-cyclohexane theta system as a function of molecular weight and concentration. For semidilute solutions we observe $D_{S} \propto N^{-2} c^{-3}$ which is in agreement with the predictions from scaling arguments for the self diffusion coefficient. On the other hand, if the cooperative mode is interpreted by $D_{c} \propto N^{x} c^{y}$, the results of $0<x<0.7$ and $0<y<0.5$ are in disagreement with scaling predictions of $D_{c} \propto N^{0} c^{1}$. The assumptions and potential shortcomings of the blob model which is used in the derivation of the power law predictions and the dynamic scattering equations is examined. In addition, we have obtained monomeric friction coefficients from the $D_{s}$ results within the framework of Doi-Edwards model. A comparison is made of the concentration dependence of the monomeric friction coefficient from the present data to that from similar experiments on a good solvent (tetrahydrofuran) system which have been discussed in last years annual report and from shear relaxation modulus measurements on the polystyrene in Aroclor 1248. 


\section{BLENDS PROCESSING}

Blends are a relatively new commercial development which represent one of the most rapidly growing fields in the U.S. synthetic polymer industry. Blending two or more polymers provides an inexpensive means of developing materials with improved properties and performance. By blending existing polymers, industry can avoid the expense of both new synthesis plants and new government approval tests. Further, properties can be tailored to performance specifications by blending. For reasons such as these, blends have already become nearly 20 percent of the U.S. production. Their use as engineering materials is projected to triple between 1982 and 1987.

There is strong industrial interest with many companies involved. General Electric has formed a new blends center and Exxon has sent a Research Associate to the Polymer Science and Standards Division to work on blends. In response to expressions of need from the plastics community, the Society of Plastics Engineers, Inc., together with its Engineering Properties and Structure Division and the National Bureau of Standards (NBS), have agreed to institute a joint program to provide data on the thermodynamics and nonequilibrium phase behavior of polymer blends. NBS will be responsible for the overall guidance of the technical aspects of the program with the aim of assuring reliability of the data evaluations, and will provide a center for coordination with other data compilation centers.

A characteristic of most blends is that they spontaneously separate into phases of different compositions. This phase separation markedly affects the useful physical properties. Industrial development of blends is hindered by an inadequate knowledge of the fundamental factors that govern the kinetics of phase separation during processing. Proper process control requires a knowledge and understanding of phase separation kinetics which are dependent on diffusional processes, interfacial phenomena, and the underlying thermodynamics. The activities of this task are focused on measurement methods, data, predictive models, and general theoretical descriptions that will form a scientific basis for optimizing control of blend processing.

NBS has five experimental facilities which, taken as whole, represent a unique combination of tools for the study of phase separation kinetics in polymer blends: small-angle neutron scattering (SANS), Forced Rayleigh Scattering (FRS), small-angle x-ray scattering (SAXS), fluorescence energy transfer techniques, and electron microscopy. The only other SANS facility in the United States is at 0 ak Ridge; there are only two other FRS instruments in the world being used to study polymeric systems; fluorescence methods are relatively new and unexploited. After adding the two-dimensional $x$-ray detector (1984), the NBS SAXS facility will be one of the finest in the world. NBS possesses state-of-the-art facilities in imaging and analytical electron microscopy.

\section{Forced Rayleigh Scattering \\ E. J. Amis ${ }^{1}$ and C. C. Han \\ 1 Research Associate, University of Wisconsin}

A Forced Rayleigh Scattering instrument has been designed. Most mechanical and optical parts have been fabricated, installed, and tested. In this technique a split laser beam is recombined at a small crossing angle on a sample which includes a trace of polymer labeled with a photochromic dye. This "writing" beam is pulsed to produce an interference pattern which bleaches a holographic grating in the sample. A second laser beam "reads" this pattern and is diffracted by the grating. As the labeled polymers diffuse in the sample, the grating is destroyed and thus, by measuring the intensity decay of the diffracted beam, the tracer diffusion coefficient of the labeled species can be obtained. Since the distance scale of this tracer method is very small (typically several orders of magnitude smaller than macroscopic tracer methods) it is possible to measure extremely small diffusion coefficients such as those expected for polymer molecules in concentrated solutions and melts. Recent theories of dynamics in concentrated solutions and melts should be tested by this experiment. Preliminary tests of the instrument have been very encouraging. Computer interface, automatic grating spacing adjustment, and temperature controlled sample chamber have been designed. This instrument can be used for in situ diffusion measurement of phase decomposition kinetics. 
H. Hasegawa ${ }^{1}$, T. Hashimoto ${ }^{2}$, H. Kawai ${ }^{3}$, C. C. Han, T. P. Lodge ${ }^{4}$,

A. J. Amis

${ }^{1}$ Assistant Professor, Kyoto University

${ }^{2}$ Associate Professor, Kyoto University

3 Professor, Kyoto University

${ }^{4}$ Assistant Professor, University of Minnesota

SResearch Associate, University of Wisconsin

Smal1 angle neutron scattering (SANS) technique is used in conjunction with small angle xray scattering (SAXS) technique to extract the "molecular scattering" from a set of "matched" lamella microphase separated polystyrene/ polyisoprene samples. This set consists of five samples with different blending ratios of (PS/PI) and (deuterated PS/PI) two block copolymers which have almost identical chain length and composition.

The periodical scattering pattern from both SAXS and SANS have been used to interpret the lamella microphase structure. The molecular scattering extracted from SANS results at different PS/PI to dPS/PS ratio and from the orientation factor obtained from SAXS show an anisotropic pattern which gives chain expansion in the direction perpendicular to the lamella interface and contraction in the direction parallel to the interface.

The expansion is expected from current theoretical understanding but the contraction has not been predicted so far.

\section{Novel Fluorescence Method for Polymer Blend Phase Diagrams}

\section{F. W. Wang and R. E. Lowry}

The raliable measurement of polymer blend phase diagrams assumes greater importance as more polymer-polymer blends are used both in the replacement of other materials and in new applications. Calorimetric and dynamic mechanical methods, which are often used for phase-diagram determination, have been found to be unreliable in some cases. Furthermore, these methods cannot be used if the glass-transition temperatures of the constituent polymers are relatively close to each other.

The fluorescence quenching method determines phase diagrams by measuring the intimacy of mixing of the constituent polymers at the molecular level. The constituent polymers are chemically labeled with either a small quantity of chromophores or a small quantity of quenchers. The fluorescence intensity of the chromophores will be reduced by the quenchers only when the chromophores and the quenchers are well mixed and separated by small distances. The fluorescence intensity will be much smaller for a single-phase blerid than for a two-phase blend. This method was successfully demonstrated for two-phase blends made from poly(methyl methacrylate) and poly(ethyl methacrylate). It was shown that addition of poiy(vinylidene fluoride) turns the above two-phase system into a single-phase system.

\section{Nanosecond Fluorimeter for Measurements of Polymer Phase Diagrams}

F. W. Wang, F. L. MeCrackin, J. D. Barnes, and W. H. Grant

The nanosecond fluorimeter for measurements of polymer. phase diagrams by the energy transfer technique is being installed by the vendor. The fluorimeter has been interfaced to the computer in a simple way.

We are now developing a computer program to treat the data from the fluorimeter. Further, we are preparing a more sophisticated interface between the fluorimeter and the computer.

\section{Installation of a Laboratory for Polymer Synthesis}

B. J. Bauer

A laboratory has been established for polymer synthesis. Polymerizations by all standard techniques can be used in sample preparation. Special emphasis has been placed on anionic 
polymerization. A high vacuum line has been designed and constructed. It is capable of production of small amounts ( $\sim 10 \mathrm{~g}$ ) of model polymers such as blocked, branched, functionally terminated, or network polymers.

\section{Digital Small-Angle Scattering Camera}

\section{J. D. Barnes}

The Polymer Science and Standards Division has identified a need for improved capabilities in the collection and analysis of small-angle x-ray scattering data. A new facility, which essentially duplicates the one at the National Center for Small-Angle Scattering (Oak Ridge National Laboratory), is being designed and constructed.

The NBS camera will use a two-dimensional position sensitive $x$-ray detector to collect data on an array of up to $128 \times 128$ picture elements (pixels) with 16-bit resolution at each pixel. The data collection hardware and software are functionally equivalent to those being employed on the small angle neutron scattering instrument in the NBS Reactor Radiation Division.

We will use an existing $12 \mathrm{~kW}$ rotating-anode generator as the $x$-ray source. The beam path supporting and positioning hardware is being redesigned to provide better mechanical stability than is found on the Oak Ridge machine. The alignment adjustments will be computer controiled in order to facilitate careful monitoring and control of these functions by the user. The monochromator system is being redesigned to eliminate the need for large movements of the beam path when changing the $x$-ray wavelength.

Given the need to observe subtle qualitative features in the data, considerable emphasis is being placed on graphical display of the data. A separate computer is being installed to provide interactive data analysis using color graphics without interfering with the data collection process. This computer will also be used to apply detector sensitivity corrections, to desmear the data, to derive correlation functions, and to calculate appropriate integrals over the corrected scattering functions.

It is expected that these capabilities will provide powerful tools for use in such studies as morphology of semicrystalline polymers, kinetics of phase transitions, deformation processes, crack formation in glassy polymers and composities, and studies of phase behavior in polymer blends.

\section{New Correlation Discovered for Surface Tension of Simple and Polymeric Liquids}

\section{C. Sanchez}

A new equation has been derived which relates the surface tension $(\sigma)$ to a liquid's isothermal compressibility $(K)$ and mass density $(\rho)$. This new correlation is

$$
\sigma=\left(A_{0} \rho / K\right)^{1 / 2}
$$

where $A_{0}$ is a constant that has some interesting properties. First, it is temperature independent in the normal liquid range (water is a notable exception). Second, it is an invariant for a wide class of organic liquids that includes aliphatic and aromatic hydrocarbons, alcohols, amines, acids, as well as other oxygen and nitrogen containing organics. It is also invariant among the diatomic elements (except hydrogen) and among the heavy noble elements. For polymeric liquids $A_{0}$ is temperature independent, but varies by about a factor of 2 among several chemically different polymers. It is remarkable that hydrogen, methane, and polyethylene all have the same value of $A$. Third, if the Lennard-Jones force constants are known for a liquid, $A_{0}$ can be estimated theoretically for this pair potential. That this correlation exists suggests that the force constants are not independent, but are coupled in a special way--a property that heretofore had not been recognized. 
The objective of this research is to provide basic materials science, engineering and test methods which may be used by sectors of the health care industry for the development of new or improved materials, delivery systems and standards.

Materials research efforts support development of materials and techniques which will provide adhesion and increased durability and wear resistance of composite restoratives, decrease marginal microleakage, and add to the knowledge available for the development and use of less costly alternatives to gold casting alloys and dental porcelains. The understanding of the structure and physical chemistry of calcium phosphates as they relate to biological systems is being advanced for the development of new or improved preventive stomatological treatments (applications for other than oral diseases are also considered). Techniques for characterization of low modulus, porous polymeric implant materials are being investigated and analyzed. Methods for modifying surgical bone cement to improve durability and performance of bone cement-implant systems are being pursued.

Dental Composite and Sealant Chemistry and Related Developments

J. M. Antonucci, G. M. Brauer, R. L. Bowen ${ }^{1}$, J. W. Stansbury, R. E Deh1, and N. W. Rupp ${ }^{1}$

\section{${ }^{1}$ Research Associate, American Dental Association}

In an effort to significantly enhance the in vivo performance and durability of dental composite and sealant materials, several types of dental resin systems are under development which are designed to yield polymers having the following desirable characteristics: (a) Minimal or zero polymerization shrinkage from monomer systems, (spiro monomers which expand slightly on polymerization also are being synthesized). Composites based on these types of dental resins should have improved marginal adaptation and adhesion to tooth structure. (b) Improved chemical stability inciuding minimal residual vinyl unsaturation. The inclusion in the resin system of relatively small quantities of multi-functional chain transfer agents (e.g., polythiols such as pentaerythritol-tetra (3-mercaptopropionate), PETMP have been shown to be effective in reducing the residual vinyl content of the polymer. Composites based on PETMP also exhibit improved esthetics and color stability. (c) Minimal sorption of water and other oral fluids. Excessive sorption of fluids by composites and sealants results in plasticization and/or chemical disintegration which contributes to a reduction in the service life of these materials. Composites based on the use of a low surface energy resins such as the highly fluorinated oligomeric multifunctional methacrylate, PFMA, with several conventional diluent comonomers (e.g., 1,6-hexamethylene dimethacrylate, 1,10-decamethylene dimethacrylate, neopentyl dimethacrylate, etc.) had good mechanical properties, improved marginal adaptation and extremely low water sorption. Composites based on PFMA also exhibit excellent esthetics and color stability. Such composites are expected to resist staining and plaque accumulation.

A method for determining the water sorption of composites and sealants by near-infrared spectroscopy was developed and appears to be a more sensitive technique for measuring water uptake than the standard gravimetric method. In an effort to improve the shelf life of dental composite and sealant materials, the use of initiator systems based on the relatively stable cumene hydroperoxide rather than the usual benzoylperoxide has been investigated. The need for a more stable ambient temperature initiator system is especially critical to the needs of the military where storage conditions are more severe than those encountered in ordinary clinical usage. It was found that PETMP is an effective promotor for the radical decomposition of cumene hydroperoxide. This initiator system offers promise for the formulation of dental composite and sealant materials of enhanced storage stability.

PETMP also has found utility in reducing the setting times of denture base materials while improving the esthetics and color stability of the cured material. When used in modest amounts in the liquid portion (e.g., methyl methacrylate) of one surgical bone cement it is effective in reducing the peak temperature of the exotherm without adversely affecting dough, and setting times or the compressive strength of the cement. A more moderate exotherm is desirable in surgical bone cement since it reduces the chance of inflammatory or other adverse responses in tissues contiguous to the cement. 
R. L. Bowen ${ }^{1}$, G. M. Brauer, J. M. Antonucci, J. W. Stansbury, D. W. Misra ${ }^{1}$,

E. Cobb ${ }^{2}$, and J. M. Cassel

${ }^{1}$ Research Associate, American Dental Association

${ }^{2}$ Guest Worker, Georgetown University School of Dentistry

Effective bonding to tooth enamel is presently achieved by means of acid-etch treatments. Similar treatments for bonding to dentin have proved ineffective, and are often contra-indicated. Adhesive systems and methodologies that would bond effectively not only to dentin but also to enamel without the necessity of removing sound hard tissue are being investigated.

Certain alkyl cyanoacrylates, which polymerize anionicaliy at ambient temperatures, had been shown to initially form strong bonds to dentin. However, these bonds weakened significantly on exposure to water. In an effort to stabilize and maintain the good initial bond strength to dentin, a functional alkyl cyanoacrylate, which is capable of crosslinking with itself and/or copolymerizing with dental resins has been synthesized. Preliminary studies have shown this cyanoacrylate to be a highly reactive monomer. Evaluation of its bonding potential to dentin will be carried out in the near future.

Another unique approach for a tooth-structure conserving bonding procedure has recently been developed which utilizes brief applications of a cleanser mordant aqueous solution of ferric oxalate, a surface-active comonomer and a coupling agent. The surface-active comonomer is either NTG-GMA, or NPG-GMA, the adducts of glycidyl methacrylate with $N(p-t o l y l)$ glycine and N(Fhenyi)glycine, respectively. The coupling agent is PMDM, the diadduct of pyromellitic anhydride and 2-hydroxyethyl methacrylate. Scanning electron microscopy evidence shows that the iron oxalate solution alters the surface layer. The surface-active comonomer and the coupling agent then provide molecules which are bound to the altered surface and yield the capability of copolymerizing with subsequently applied resin of the composite material. With enamel surface the average tensile bond strength was $13.5 \mathrm{MPa}$, about the same as obtained with the acid-etch technique. With dentin, tensile bond strength averaged $13.1 \mathrm{MPa}$ (range, 10.2 to $18.2 \mathrm{MPa}$ ). In addition to an adhesive mode of failure, the fractured surfaces exhibited frequent cohesive failure in the composite and occasional cohesive failure in dentin. The biocompatibility of this promising adhesive bonding system is undergoing evaluation prior to clinical trials.

\section{Dental Cements}

\section{G. M. Brauer, J. M. Antonucci, and J. W. Stansbury}

Dental cements with good biocompatibility, improved mechanical properties and resistance to erosion on exposure to the chemical environment are needed. With the exception of cements based on aqueous solutions of poly(acrylic) or similar poly(alkenoic) acids none have shown significant adhesion to tooth structure. Because of the hydrophilic character of these latter cements, there is some question of their long term durability in the oral cavity, especially with patients having hyperacid oral conditions.

Two approaches have been initiated to develop more hydrophobic, tough cements with adhesive potential to various substrates.

One approach is based on enhancing the strength and chemical resistance of current cements based on zinc oxide-eugenol (ZOE)-2-ethoxybenzoic acid (EBA) by replacing the eugenol with esters of vanillic acids. Cements based on n-hexyl vanillate (HV) and EBA have been shown to have higher strength and lower solubility than currently used EBA-ZOE cements. In contrast to ZOE containing cements, the HV-EBA cements do not inhibit free radical polymerization, adhere strongly to non-precious metals, porcelain, and composites, and can be formulated with methacrylate monomers to yield composite cements of even higher strength and lower solubility. Work to modify the newly developed hexyivanillate (HV), o-ethoxybenzoic acid (EBA), and zinc oxide ( $\mathrm{n} O$ ) dental cement for intermediate restorative applications has led to the synthesis of several new types of vanillate esters. Substitution of the difunctional decamethylene divanillate for the monofunctional HV in a cement formulation yielded stronger and less soluble materials. Enhanced physical properties were also obtained for cements which included methacryloylethyl vanillate; a compound capable of free radical induced polymerization as well as the normal Zno chelating reaction. Hybrid cement 
composites arising from powders containing $\mathrm{ZnO}$ and benzoyl peroxide treated glass along with liquids consisting of HV-EBA and various monomethacrylates have been evaluated. These formulations yielded cured cements which exhibit extremely high strength and low solubility. In addition, they demonstrated excellent adhesion to a wide range of substrates.

The incorporation of esters of syringic acid, a.potential caries reducing agent, as a complete or partial replacement for HV in cement formulations has been investigated. Cement products obtained with Zno have had more than adequate physical properties, however, a study of any cariostatic qualities of these cements remains to be done.

To date, all of the preliminary biocompatibility results have indicated that hexyl vanillate elicits equivalent or milder tissue responses than eugenol.

These newly developed materials show great promise as intermediate restoratives, luting base, endodontic cement, crown and bridge cement and as a cement for repairing fractured porcelain. The second appraoch to a more durable dental cement is based on the development of nonaqueous polycarboxylate cements.

Conventional polycarboxylate cements are based on the reaction of aqueous solutions of poly(acrylic acid), or similar poly(alkenoic acids), with a basic inorganic powder (e.g., zinc oxide, magnesium oxide, ionomer glasses). The acid-base reaction that occurs on admixture of the aqueous polyacid and the base powder yields ionically crosslinked polymeric matrices that are rigid and hydrophilic in nature. The feasibility of obtaining less rigid and more hydrophobic dental cements by the reaction of the commercially available dimer (DA) and trimer (TA) acids with a variety of basic powders (e.g., $\mathrm{Ca}(\mathrm{OH})_{2}, \mathrm{ZnO}$, etc.) is being explored. DA and TA are moderately viscous liquid polycarboxylic acids of high molecular weight possessing a unique chemical structure of a bulky, flexible, hydrophobic core which terminates in 2 or 3 hydrophilic carboxyl groups $\left(-\mathrm{CO}_{2} \mathrm{H}\right)$, respectively. The relatively low carboxyl content, large size hydrophobic nature of these acids should result in dental materials of excellent biocompatibility.

Cements having clinically acceptable setting times, and good physical and mechanical properties were obtained from base powders containing calcium hydroxide, magnesium oxide and zinc oxide. DA admixed with $\mathrm{Ca}(\mathrm{OH})_{2}$ in a powder/liquid ratio (by weight) of 1.5 gave a fast-setting cement ( $3 \mathrm{~min}$.) with 24-hr compressive (CS) and diametral tensile strengths (DTS) of 25 and 4 MPa, respectively. This $\mathrm{DA}-\mathrm{Ca}(\mathrm{OH})_{2}$ cement had a low water solubility of only 1.5 percent after 24 hours at $37^{\circ} \mathrm{C}$; the water after this period of storage was alkaline (a condition which should protect pulpal tissues from acid attack and stimulate secondary dentin formation). Under compressive loading (crosshead speed $=1 \mathrm{~mm} / \mathrm{min}$ ) specimens of this cement do not fracture but show significant deformations. A $\mathrm{TA}-\mathrm{Ca}(\mathrm{OH})_{2}$ cement behaved similarly. The addition of 17 percent tricalcium phosphate to a $\mathrm{DA}-\mathrm{Ca}(\mathrm{OH})_{2}$ formulation $(\mathrm{P} / \mathrm{L}=2)$ gave a material that hardened in $7 \mathrm{~min}$. and deformed only slightly under the same compressive stress $(C S=32$, $D T S=6 \mathrm{MPa})$. The absence of brittle failure is indicative of the non-friable nature of these cements. Thus far, the strongest $\mathrm{DA}-\mathrm{Ca}(\mathrm{OH})_{2}$ cement was one consisting of a base powder with equal parts of $\mathrm{Ca}_{3}\left(\mathrm{PO}_{4}\right)_{2}, \mathrm{Ca}(\mathrm{OH})_{2}$, and $\mathrm{MgO}(\mathrm{P} / \mathrm{L}=2)$; $\mathrm{CS}=41$; $\mathrm{DTS}=6$ $\mathrm{MPa}$. The energy absorbing nature of all these $\mathrm{Ca}(\mathrm{OH})_{2}$ cements coupled with their low solubility and alkaline character suggests their use as cavity liners, endodontic sealers, impression materials, etc. The strength and solubility characteristics of these $\mathrm{Ca}(\mathrm{OH})_{2}$ cements are superior to those currently available.

$D A$ and $T A$ with micronized zinc oxide $(P / L=7)$ yielded tough, extremely hydrophobic cements (setting time $=7.5 \mathrm{~min}$ ) $C S=50$, DTS $=7 \mathrm{MPa}$ ) which may find use as temporary and intermediate restoratives. Because these cements do not inhibit the polymerization of composites they are amenable to the formulation of hybrid composite-cement materials. The setting mechanism of these novel polycarboxylate cements involves primarily chain extension through the formation of multidivalent metal carboxylate linkages. The versatile nature of cementitious materials obtained from $D A$ and TA with different base components suggests a wide range of potentiai dental applications.

\section{Wear Mechanisms and Durability of Dental Composite Materials. Effect of Degree of Cure}

W. L. Wu, J. E. McKinney, E. E. Debelius, and J. M. Cassel

Since clinical evaluations on dental restoratives require three to five years, it is necessary to develop and utilize appropriate laboratory tests to obtain reliable durability assessments in a 
reasonable period of time. Microdefect analyses involving a photographic silver staining process and small angle $x$-ray scattering reveal the extent of damage in both in vivo and in vitro worn materials. On in vivo worn restoratives the resuits from these techniques revealed subsurface damage on both stress and nonstress bearing surfaces. Accordingly, it is believed that the oral chemical environment plays a very important role in the degradation of composite restorations. Earlier laboratory tests have shown that the wear resistance decreased markedly after certain food simulating liquid chemicals diffused into the composite specimens. In particular, those presoaked in a 75 percent/25 percent ethanol-water solution revealed the greatest extent of subsurface damage and wear (pin and disc). At this concentration the solubility parameters of the solvent and BIS-GMA polymer (used as matrix) are assumed to be proximate.

FTIR measurements revealed a degree of cure of only about 65 percent for a BIS-GMA-TEGDMA polymer cured at the in situ temperature $37^{\circ} \mathrm{C}$. Increasing the degree of cure is expected to improve the wear resistance and durability of the composites. In recent work the degree of cure was increased by elevating the cure temperature. Although this method may not be practical for in situ restorations, it affords an evaluation of the influence of the degree of cure on material properties. Wear and hardness measurements were conducted on three kinds of commercial dental composites: conventional (large particle reinforcing filler), visible light activated, and a microfill. Specimens cured at temperatures up to $80^{\circ} \mathrm{C}$ were soaked in water and the 75 percent/25 percent ethanol-water solution for one week. Subsequent wear and hardness measurements on the water soaked specimens revealed no significant changes with cure temperature. With the ethanol soaked specimens, however, considerable improvement with increasing cure temperature was observed in the wear and hardness of all of the composites. With the $80^{\circ} \mathrm{C}$ cured specimens, no significant difference was observed between wear of the water and ethanol soaked specimens.

With cross-linked polymers the degree of cure is apparently limited by the elevation of the glass transition temperature $\mathrm{Tg}$ during the curing process. At a certain point during the cure the viscosity becomes so large that the reactive groups cannot become proximate and are used up in other way's, thus terminating the cure. In order to obtain more knowledge of the behavior of this process, we have initiated a series of low frequency vibration measurements to follow the isothermal curing of dental polymers at different temperatures. Companion FTIR measurements will be made to relate Tg to the degree of cure. The information obtained from these measurements will be used to evaluate the potential of new dental polymers under development at NBS.

The diffusion processes of water and other food simulating chemicals into dental composites have been monitored quantitatively using a CAHN electrobalance. Fickian type diffusion is observed for water as a penetrator, and the diffusion coefficient of water is not suppressed by the presence of the fillers. Class-II diffusion is found for other liquids which swell the dental resins. The presence of fillers in this case decrease the extent of swelling significantly.

\section{Porcelain Fused-to-Metals and Dental Ceramics}

J. A. Tesk, W. deRijk 1 , H. R. Kase ${ }^{2}$, G. Widera ${ }^{3}$, D. L. Menis ${ }^{1}$, and

R. W. Hinaan 4

1 Research Associate, American Dental Association

${ }^{2}$ Guest Worker, University of Gottingen, W. Germany

${ }^{3}$ Guest Worker, University of Illinois, Chicago, IL

${ }^{4}$ Guest Worker, U. S. Navy

Porcelain fused-to-netal (PFM) restorations often fail by cracking of the porcelain or delamination of the porcelain veneer. A major cause of this failure is thought to lie in the thermomechanical induced residual stress from firing of the system at high temperatures. Another source of failure lies in attainment of a poor porcelain-to-metal bond; this can frequently be attributed to a lack of proper process control (technique variations) and/or to lack of a chemical compatibility between alloy and porcelain.

To characterize the PFM bond strength, a four point bending beam composite (BBC) has been developed. Simple BBC theory is utilized to calculate failure stress. For modulus ratios of two, (approximately that of metal to porcelain for nonprecious alloys) the calculated stress is within 6 percent of that calculated from a finite element model of the system. Up to four interfaces are provided for PFM bond failures. Fractures occur within porcelain or at interfaces. It has been found that a cumulative failure plot using Weibull distribution analysis shows clear distinction 
between systems; such interactions are not significantiy apparent from the means of failure strengths alone. As the system and analysis is developed it is expected that industry will begin to adopt it as it provides more information with fewer samples in less time than other tests currently employed and is capable of characterizing PFM systems according to several parameters.

To characterize the residual stress of PFM and, therefore, stress compatibility, the change in gap of a porcelain veneered split metal ring is being employed. The rationale for this has been developed. A finite element model which describes the gap as a function of thermal history and material properties is nearing completion. Ultimately, it is expected that the ability to calculate gap changes which correspond to experimentally determined changes will lead to definition of the stress within the system. Correlation with clinically/experimentally observed failure of the porcelain is expected to lead to delineation of the limits of stress which can be imposed on each combination of porcelain and alloy. Use of a tensile stress failure criteria should also provide a means for calculation of thermal expansion compatibility between porcelain and alloy, thereby providing a method which may be used by industry for development of new materials/techniques, quality control and for use in development of standards.

An important consideration in calculation of thermal stress compatibility is the elastic modulus of the materials involved and their temperature dependencies. The moduli of porcelain have been determined and hysteresis between heating and cooling shows evidence of some microcracking. Generally, the modulus of the porcelain decreases about 1 percent per each $100{ }^{\circ} \mathrm{C}$ increase in temperature with moduli $\sim 70 \mathrm{GPa}$ near $23^{\circ} \mathrm{C}$.

New compositions of ceramic materials that can function as veneers for dental prostheses have been formulated. These materials are formed from solutions. Fusion temperatures in the $710{ }^{\circ} \mathrm{C}$ to $750{ }^{\circ} \mathrm{C}$ have been obtained. Several of these materials have adequate thermal expansion and good chemical durability.

Mathematical analysis has shown that the fusion temperature of the modified aluminosilicates can be predicted as a function of the constituent oxide concentrations.

In addition it has been found that the firing of dental porcelains beyond the mature fire does not increase chemical durability.

\section{Dental Casting Alloys and Investments}

J. A. Tesk, R. W. Waterstrat ${ }^{1}$, S. Hirano2, R. W. Hinman ${ }^{3}$, H. R. Kase ${ }^{4}$, W. deRijk ${ }^{1}$, and $H$. Argentar ${ }^{5}$

1 Research Associate, American Dental Association

2Guest Worker, Tsurumi University, Japan

3Guest Worker, U. S. Navy

4 Guest Worker, University of Gottingen, W. Germany

SPrivate Collaborator

Because of superior properties and/or lower costs, dental alloy systems other than gold are being used and introduced at a high rate. Most of the newer alloys are based on nickel and an increasing percentage of palladium based alloys are appearing. Although the use of nickel based alloys has not to date produced an obvious problem of biocompatibility, concern over potential latent carcinogenecity and allergic sensitization has not abated. For this reason, titanium based alloys offer some characteristics which are appealing as they may be expected to be non-toxic, durable and strong. A major impedment to their widespread use lies in the need to find a way to successfully cast these alloys under dental laboratory conditions. Recent work at NBS with magnesium oxide investments has produced a successful casting which has not been unduly contaminated by the investment, as evidenced by the surface finish and non-brittle nature. The production of a series of castings for evaluation of clinical potential will be undertaken in the near future.

As the desire for new alloys continues, a method for evaluating the castability and the effects if element additions as functions of alloy casting temperature and mold temperature is needed to aid i. erocessing quality control and development.

A simple technique has been developed for these purposes; it involves the use of a polyester ipl 1 pattern. The percentage of completely cast grid segments is used as a castability value, $C_{v}$. 
Because the standard deviation (SD) of $C_{v}$, is a function of $C_{v}$ it is convenient to use a transform of $C_{v},\left(C_{v t}\right)$ to mathematically represent $C_{v}$ as a function of temperature. It has been found that for six alloys investigated, the same function is capable of describing $C_{v t}$ at the 99.5 percent confidence level.

$$
c_{v t}=a+b T_{A}^{12} T_{M}^{2}
$$

where $a$ and $b$ are constants, characteristic of each alloy

$T_{A}=$ alloy casting temperature in terms of superheat above the solidus;

$T_{M}=$ the mold temperature in degrees celcius

The relationship between $C_{v t}$ and $C_{v}$ is

$$
c_{v t}=\ln \frac{2 / 3+\sqrt{c_{v} / 100}}{2 / 3+\sqrt{1-c_{v} / 100}}
$$

A graph of $C_{v t}$ vs $C_{v}$ provides a convenient way of obtaining the inverse transform.

The next step in the program is to represent the contribution of various elements to castability, as a function of temperatures.

\section{Dental Chemistry, Crystallography, and Preventive Treatments}

W. E. Brown ${ }^{1}$, L. C. Chow ${ }^{1}$, M. Mathew ${ }^{1}$, S. Takagi ${ }^{1}$, M. Tung ${ }^{1}$, G. L. Voge ${ }^{1}$,

T. M. Gregory ${ }^{1}$, C. M. Carey ${ }^{1}$, B. Sieck ${ }^{1}$, and C. Schreiber ${ }^{1}$

${ }^{1}$ Research Associates, American Dental Association

Fundamental studies on the chemical properties and crystallographic structures of tooth and bone minerals and other biologically important calcium and phosphate containing compounds are conducted. The information obtained is applied to the development of improved caries preventive techniques. Work conducted under this project includes studies on topical fluoridation, mechanisms of biomineralization, caries mechanisms, calcium phosphate cements, crystal structures of biominerals, and $x^{-\infty}$ ray magnification microradiography.

Work continues on the new procedure for topical fluoridation which involves treating the tooth with solutions that form dicalcium phosphate dihydrate (DCPD) in the enamel to make it more reactive towards fluoride. A cooperative fluoride-uptake and caries-score study was carried out with investigators at NIDR using the rat model. The DCPD pretreatment considerably increased the permanently-held fluoride content of teeth and produced the lowest caries score. However, due to the relatively large standard deviations, the caries score was not significantly different from the fluoride treatment alone. Our present procedure requires two treatments, DCPD-forming solution followed by fluoride solution. By incorporating into the DCPD-forming solution a complex fluoride which does not react with calcium ions, the procedure may be reduced to a single treatment. Initial laboratory results showed that $\mathrm{SiF}_{6}{ }^{2}$ ions served well for this purpose. Based on the above principle, a patent application was filed: "Composition and Methods for Topically Fluoridating and/or Mineralizing Dental Enamel."

We have long advocated the view that octacalcium phosphate (OCP) participates as a precursor in the formation of tooth and bone mineral. There is growing evidence that in biological calcifications OCP is formed first, then hydrolyzes to a nonstoichiometric hydroxyapatite. A procedure was developed in our laboratory by which these two processes can be separated and studied individually to determine the effects of physiologically active components on the rates of these two mineralization processes which form tooth, bone, and pathological mineral deposits such as those in atherosclerotic plaque.

Caries Mechanisms: The work on caries mechanisms has been expanded to include studies on four levels: (1) theoretical considerations which focus on the fact that the relative rates of diffusion of ions into and out of the lesion can be important parameters affecting the rate of caries 
progression, (2) bench-scale experiments using a two-compartment diffusion cell to test the major principles of the proposed caries model, (3) a computer simulation model which has produced results in close agreement with the bench scale studies and allows us to survey rapidly the effects of a large number of variables, and (4) ultramicro-analytical studies on tooth sections to establish quantitatively the events occuring within the enamel during artifically induced caries formation. Results from the above studies have produced important new insights into the factors governing the caries process.

Progress has been made on the development of cements that harden in water and form compositions nearly identical to tooth and bone mineral. A method was developed which can produce cement specimens with greater strengths and with pores if needed for bone implant applications. In collaboration with the Research Institute, a study to test the biocompatibility of the cement using animal models has been initiated. A patent application, "Combinations of Sparingly Soluble Calcium Phosphates in Slurries and Pastes as Mineralizers and Cements", was filed.

The crystallographic studies provide a structural base for understanding the chemistry of biominerals and their interactions with dental materials. Crystal structures of $\mathrm{Ca}_{2} \mathrm{KH}_{7}\left(\mathrm{PO}_{4}\right) \cdot \mathrm{H}_{2} \mathrm{O}$ has been determined using $x$-ray and neutron diffraction data. Research on the structural studies of octacalcium phosphate, $\mathrm{Ca}_{8} \mathrm{H}_{2}\left(\mathrm{PO}_{4}\right)_{6} \cdot 5 \mathrm{H}_{2} \mathrm{O}$ and its role in biomineralization and the surface and colloidal properties of apatites continue to be a major activity. .

A new study of the structural features in mineralized tissues using $x$-ray magnification microradiography has been initiated. $X$-ray magnification microradiography is a new technique that was developed by the National Bureau of . Standard's Metallurgy Division. Considerably better resolution may be obtained by this technique than by standard contact microradiography. Preliminary studies have clearly shown images of dentin tubules and special features of carious lesions.

Clinical and Laboratory Investigation of Dental Amalgams, Composites and Glass Ionomer Cements

N. W. Rupp ${ }^{1}$, G. C. Paffenbarger ${ }^{1}$, and R. W. Waterstrat ${ }^{1}$

${ }^{1}$ Research Associates, American Dental Association

The success or failure of dental restorations can be determined only after long term, greater than two years, observations. The causes of failure relate to three categories: (1) the physical, mechanical and chemical properties of the material; (2) the operator's selection of material, cavity preparation and manipulation of the material; and (3) the patient's home care. Sorting out of the factors contributing to the durability of restorations requires a careful combination of clinical studies and laboratory analyses.

Amalgam restorations placed in our clinical study over nine years ago are continually being observed. These, along with observations of in vitro specimens made six years ago, show an interesting correlation within our laboratory and with observations reported from other laboratories. Unrestricted amalgam specimens, $4 \times 8 \mathrm{~mm}$ cylinders, have been stored at $60^{\circ} \mathrm{C}$ for six years and their iength and diameter measured and changes in dimensions recorded. Also, restricted specimens have been compacted in cavities in a steel block, also stored at $60^{\circ} \mathrm{C}$ for six years, and the extrusion from the cavities measured. Currently we have the clinical data from an outside laboratory for three alloys in common with our study. The clinical study reports the deterioration of restorations directly in comparison with our yearly laboratory observations. When this data, after six years, is plotted, there is a straight line from the fiducial reading. We are now searching for clinical reports on other alloys on which we have data to determine if the laboratcry data is consistently compatible. Also, we are studying the data to determine the shortest or earliest time period which can be observed in the laboratory that will predict long-term deterioration of restorations. Clinical studies are expensive and time consuming. If the laboratory test in a few months does predict what the long-term clinical studies finally show, then patients, dentists, and manufacturers will be saved considerable moriey and time.

Composite restorations placed both in vivo and in vitro are being observed for margin staining using commercial products recommended for bonding to dentin. Only those products having proof of safety to the pulp are being used in vivo. Currently two brands of dentin bonding agents indicate adequate bond strength to prevent gross margin leakage. When this is statistically confirmed, the results will be published. This is necessary because the gingival margin of a composite restoration rarely has sufficient enamel for bonding; thus the gingival margin is the first area to break down 
with stain and/or caries. As soon as the dentin bonding agent being developed in this laboratory (Dr. R.L. Bowen) is determined to be biologically safe to use, it will be incorporated in a clinical study.

Glass ionomer cement restorations are being placed in selected cavities. Some restorations have been retained for over two years. This material can be used in gingival erosion cavities that are extremely sensitive without grinding or removal of tooth tissue. The sensitivity is immediately reduced and the restoration appears to be effective. The handling technique is extremely difficult and is also being studied.

\section{Characterization of Porosity in Porous Polymeric Implant Materials}

\section{R. E. Dehl}

Porous synthetic bicmaterials are currently being used as implants, where they become stabilized by the ingrowth of hard or soft natural tissues. Both the rate and the type of tissue ingrowth are affected by the porosity of the materials, and it is important to be able to characterize the porosity by reliable and reproducible methods. In response to a request from the office of Medical Devices, Food and Drug Administration, a study is being conducted by the currently existing methods for characterizing the porosity of two commercially available porous polymeric implant materials, porous polyethylene and a composite of polytetrafluoroethylene and carbon.

In previous years, studies of the porous implant materials included measurements of the pore volume and interconnecting pore size by mercury porosimetry; a quantitative analysis of the stress-strain behavior of both materials, as it might affect the porosity measurements; and an estimation of the average pore "diameter" from measurements of the ratio of the specific pore volume to the specific surface area. Error analysis of the mercury intrusion experiment revealed increasing accuracy of the measurement with increasing pressure, corresponding to smaller pores. The shape of the volumeweighted pore size distribution curves were found to be approximately log-normal.

A major effort is now directed to the measurement of pore volume and pore size by quantitative microscopy (stereology). Samples were prepared for optical analysis by filling the porous materials with a liquid resin which hardened to allow the surface to be ground and polished. Under intense overhead illumination, both materials revealed differences in reflectivity between the pore (resin) phase and the polymer phase, thus permitting quantitative microscopic analysis of the pores. Thirty photomicrographs of each of the materials, taken at random spots on the surface, were analyzed using a transparent overlay grid. The pore volume fraction was determined by measuring the fraction of grid intersects or "points" $\left(P_{p}\right)$, and the mean intercept length, a measure of pore "size", was determined from the ratio of $P_{P}$ to $N_{L}$, the number of pore line intercepts per unit test length.

A discussion has been prepared concerning the advantages and disadvantages of each of the porosity characterization techniques. The accomplishment of this project's objectives will be of significance to the medical device industry and medical implant standards development, as well as to the users of porous implant materials.

\section{Surgical Bone Cement Modification}

\section{G. M. Brauer, J. A. Tesk, J. W. Stansbury, W. L. Wu, and J. E. Mckinney}

Surgical bone cement, while serving a necessary function in orthopedic practice, has several shortcomings which become especially apparent in total joint replacements which require large amounts of material. Improved biocompatibility, low exotherm temperatures during polymerization, and more stable interfaces with bone and implant materials are some of the problems addressed in this investigation.

In comparison with the commercial, surgical simplex material, bone cements with shorter setting times, lower peak exotherms, and higher compressive strengths have been developed. With strength values unaffected, maximum temperatures were significantly reduced for formulations containing small quantities of pentaerythritol tetra (3-mercaptopropionate). A lowering of the peak exotherm was also accomplished by the addition of high molecular weight methacrylate monomers such as dicyclopentenyloxyethyl methacrylate: 
Radiopaque bone cements have been obtained through incorporation of pentabromophenyl methacrylate as a comonomer in the powder portion of the formulation. Cements formulated with 5 to $10 \%$ of the brominated methacrylate are expected to have acceptable dough and setting times and the copolymer should have improved physical properties since this eliminates the weakening effect of the barium sulfate presently used as radiopaquing agent.

\section{Dental and Medical Mateirials Standards}

J. M. Cassel, J. A. Tesk, G. M. Brauer, N. W. Rupp ${ }^{1}$; and W. Derijk ${ }^{1}$

${ }^{1}$ Research Associates, American Dental Association

Developments of standards for dental and medical materials is strongly supported by activities on committees and subcommittees of ANSI MD 156 (Dental Materials, Instruments and Equipment) and ASTM F-4 (Medical and Surgical Materials and Devices). Leadership roles are held as chairpersons of ANSI MD 156, ASTM F-4, the USA TAG for ISOTC 106, Task group convenor for several working groups of ISO TC 106 and by participation on the Executive Committee of the Medical Device Standards Management Board of ASNI. Numerous standards have been reviewed, edited, revised and reaffirmed for ANSI/ADA and ISO including standards for dental units, chairs, abrasives, burrs and alloys. 
The rapid growth in the use of polymeric materials derives from substitution for other materials, the expanded need due to population and economic growth, as well as new applications due to the unique properties of these materials. Long-term mechanical performance is important in critical applications. The realization of increased utilization is often limited by the lack of data and models upon which to design for long-term use. Thus, the lack of adequate test methods and data for evaluation of long-term mechanical performance not only restricts applications, but also leads to over design which results in more expensive items and limits weight savings. Evaluation and control during processing of material parameters that affect long-term performance should lead to more durable materials and, hence, reliable performance for the intended service life.

The objective of this task is to provide data, test methods, and standards for evaluation of long-term mechanical performance of polymeric materials. The approach is to identify and analyze principal failure mechanisms of commercially important thermoplastics under mechanical stress in the use environment, to determine the effects of chemical structure and fine structure morphology on mechanical properties and failure processes, and to develop constitutive relationships and models for mechanical properties to serve as the basis for accelerated test methodology and lifetime prediction. In addition, the influence of processing conditions on structure and morphology are elucidated.

The approach to development and verification of predictive models of mechanical performance are: (1) establish dependence of the principal failure modes of inhomogeneous deformation and crack initiation and growth on mechanical stress use environment and molecular structure and fine texture; (2) identify the principal molecular processes associated with deformation and failure and relate failure to molecular structure and fine texture; (3) evaluate the kinetic theory of bond rupture as a model for degradation of mechanical properties and for extrapolation of short-term measurements to predict long-time performance; (4) on the basis of (1), model failure by neck formation and propagation using time-dependent constitutive relations that predict the onset of inhomogeneous deformation:. from basei ine mechanical properties characterization; and (5) expand fracture mechanics approach to viscoelastic materials to modelcraze and crack growth phenomena.

The National Bureau of Standards has established joint programs on mechanical performance with other Federal agencies which are fostering the application of polymeric materials in areas of national concern. For example, a joint program with the Defense Advanced Research Projects Agency (DARPA) is concerned with development of molecular characterization techniques for mechanical degradation as the basis for nondestructive evaluation methods. Joint projects are executed with industries. An example is the project with Firestone Tire and Rubber Company on the effects of processing on polyethylene terephthalate (PET) fiber structure and properties.

\section{Molecular Processes Associated with Mechanical Deformation and Failure}

B. M. Fanconi, D. L. VanderHart, S. J. Kryder, J. R. Havens, and

K. L. DeVriesi

${ }^{1}$ College of Engineering, University of Utah

The elucidation of molecular processes and structure changes accompanying mechanical deformation and failure not only provides the basis for development of test methods for long-term performance and quality control, but also provides insight into the selection, processing, and design of materials with improved mechanical performance and durability. The semicrystalline structures found in some commonly used polymeric materials present significant challenges to characterization of molecular structure. Spectroscopic techniques involving ${ }^{13} \mathrm{C}$ nuclear magnetic resonance (NMR) and FTIR spectroscopies are used to elucidate molecular conformations in disordered material. Reorganization of polymer molecules during deformation affects mechanical performance and identification of the molecular processes involved and the physical structures which result are the aim of the studies.

FTIR is used to determine relationships between the number of chain scissions produced by mechanical stress, stress history, the useful service life, and molecular weight and its distribution. 
Previous work on ultra high molecular weight polyethylene (UHMWPE) had established that mechanical failure was associated with a critical concentration of chain scissions [1]. The number of chain scissions was determined from changes in end group concentrations as found from infrared spectroscopic analysis. During the past year, the effects of the initial molecular weight and the fatigue test frequency on the number of chain scissions at failure have been examined. In addition, the relationship between the growth in end group concentration and fractional lifetime has been established.

Spectroscopic investigations have been carried out on a second UHMWPE with a manufacturer's reported molecular weight of $2 \times 10^{6}$, or one-half the value of the material used in the aforementioned study. For this material it was found that failure occurred at a mechanically induced chain rupture concentration of about $1 \times 10^{18} / \mathrm{cc}$ instead of $2 \times 10^{18} / \mathrm{cc}$. This finding suggests that the original molecular weight is a determining factor in the level of molecular damage that can be tolerated before failure occurs. Whether the relationship between initial molecular weight and concentration of chain ruptures at failure is linear needs to be verified by further experiments.

The effect of fatigue test frequency on the concentration of chain scissions at failure has been investigated. The increase in chain scission concentration with test frequency is similar to the observed $[2,3]$ increase in free radical concentration at failure of nylon- 6 fibers with increasing strain rate. The latter results were interpreted in terms of competing processes. At high strain rates relaxation times for slip or uncoiling of molecules may be too slow to relieve over-stressed molecular segments with the result that more chain scissions occur. A competing process is the higher rigidity at the higher strain rates which means a smaller strain at fracture, and fewer over-stressed chains. Alternately, the weak dependence on test frequency may be caused by a slight rise in sample temperature as the fatigue frequency increases. Although according to the kinetic theory of chain scission the probability of chain rupture increases with increasing temperature the critical concentration would also be expected to rise due to a greater probability for free radical termination reactions as a result of more molecular mobility. The extent of free radical propagation reactions would decrease at higher temperatures and damage zones formed would not be of sufficient dimensions to coalesce into the microvoids that are the precursors to macroscopic failure.

The finding that macroscopic failure occurs at a critical concentration of chain ruptures suggests that monitoring the buildup in end group concentrations may serve as the basis for lifetime prediction. In order to assess the potential of this approach the increase in chain scission concentration during the course of a fatigue-to-failure test has been examined.

In this experiment the test specimens were subjected to a predetermined number of stress cycles, removed from the mechanical tester, and placed in the interferometer for infrared spectroscopic analysis. The specimens were then returned to the mechanical tester for another series of stress cycles, and the entire process repeated until the specimens failed. During the initial stress cycles the chain scission concentration increased only slightly. This was followed by a rapid rise to about 60 percent of the concentration at failure after which it rose linearly with the number of stress cycles until macroscopic failure.

The growth in the chain scission concentration with the number of stress cycles has been measured for specimens fatigued at 0.002 and $0.01 \mathrm{~Hz}$ and a peak stress of $35 \mathrm{MPa}$. These experiments yielded a similar behavior with fractional lifetimes. Thus, the general features of the rise in chain scission concentrations appear to be independent of the fatigue test frequency.

The fatigue experiments that have been carried out are constant peak stress tests. During the fatigue experiment the initialiy isotropic test specimen is elongated and preferential chain axis orientation in the direction of the applied stress occurs. The sample morphology is transformed from spherulitic texture to fiber morphology. The number of highly stressed molecules would be expected to change as these morphological changes occur. Thus, the rise in chain scission concentrations may correlate more directly with the imposed strain than the number of stress cycles in a fatigue experiment. Furthermore, the behavior of chain scission concentration with the first few cycles in fatigue is very similar to the strain induced increase in free radical concentration [2], or degradation of molecular weight [4]. The dependence of the chain scission concentration on strain has been examined. No discernible change in the aldehyde band $\left(1735 \mathrm{~cm}^{-1}\right)$ is observed up to draw ratios of about 5 . The polymer specimens were strained in a manualiy operated stretching frame 
and it has not been possible to strain at levels which would produce chain scissions without fracturing the tensile specimen. After fracture, a sizable increase is observed in the $1735 \mathrm{~cm}^{-1}$ band indicative of the occurence of chain scission processes.

[1] Fanconi, B. M. Annual Report 1982, Polymer science and standards division. Nat. Bur. Stand. (U.S.) NBSIR 82-2607, March 1983.

[2] Kausch, H. H.; DeVries, K. L. Int. J. Fract. 11: 727; 1975.

[3] Williams, M. L.; DeVries, K. L. Proc. of the Fifth Int7. Congress on Rheology, 3: S. Onogi, ed. Tokyo: Univ. of Tokyo Press.

[4] Shen, L. N. J. Poly. Sci. Poly. Lett. Edn. 15: 615; 1977.

\section{Examination of Drawn PET Fibers by Solid State NMR}

The motivation of this joint project with Firestone Tire and Rubber $C_{0}$. is to better understand molecular organization in polyethylene terephthalate (PET) fibers so as to effect better control of fiber shrinkage during processing in tire reinforcement applications.

Two questions are under current investigation:

The first is related to the presence of tie chains which are important in understanding the modulus of these materials since these are the links which connect the crystals. In particular, the sensitivity of ${ }^{13} \mathrm{C}$ NMR techniques to the structure and mobility of such chains is under investigation.

In a matrix of ordered as well as disordered chains, criteria must be established which differentiate NMR signals from tie chains from those of other structural components. This is not a straightforward problem. Nevertheless, the following criteria were adopted:

(a) Chain orientation close to the draw direction

(b) Mobility in excess of crystalline chain mobility

(c) Spatial proximity to the non-crystalline or disordered chains

In the ${ }^{13} \mathrm{C}$ spectrum (non-spinning), resonance positions indicate orientation, excess mobility is indicated by the rotation of the aromatic rings in the polymer backbone (about their 1,4 axes), and a crude proton rotating frame relaxation selection (in the presence of spin diffusion) indicates whether these mobile, oriented chains are primarily found in regions of orientational disorder. The chains which satisfy all three criteria are a minor fraction of the total number and annealing diminishes the number of chain segments having these characteristics. Possible interpretations of this diminishing population are (a) incorporation of tie chains into growing crystals, (b) densification of molecular packing in the non-crystalline regions, and/or (c) relaxation of orientation with annealing. Since the amount of orientational relaxation is small according to the spectra, process (c) cannot be the dominant one. Each of these explanations, however, ought to create its own signature on the modulus.

The second emphasis in the PET studies is to examine morphological changes which occur upon annealing. Spin diffusion measurements using proton NMR give information about domain size, particularly when domains are in the 4 to $10 \mathrm{~nm}$ size range as they are believed to be in these PET fibers. Last year preliminary studies of spin diffusion were carried out. This year, largely due to the efforts of John Havens, a post-doctoral associate, multiple pulse experiments have been initiated. In these experiments, which are experimentally very difficult, spin diffusion can be manipulated by turning it on and off. The thinking behind these experiments is that sharp magnetization gradients can be prepared-between regions of different relaxation times by allowing relaxation to occur while quenching spin diffusion. Then with a well-defined initial condition, spin diffusion is allowed to occur for different periods of time. Finally, after these times, the status of the system can be monitored without spin diffusion occurring, so that the status of the system does not change during "read out." This version of the experiment is much superior to former efforts on PET. Early results tend to verify what was observed in a more qualitative way before, namely, that the domain sizes are not changing by more than 40 percent upon post annealing. These results support the idea that extensive solid-state chain mobility does not exist during annealing in PET as it does in polyethylene, for example. Thus efforts at controlling shrinkage, i.e., controlling morphology, are better focussed on the initial solidification and crystallization process. 
Work in collaboration with Ian Ward at the University of Leeds and Dr. Bernard Meurer from CRM, Strasbourg, France, is concerned with drawn and undrawn polyethylene. Efforts were focussed on measuring ${ }^{13} \mathrm{C}$ longitudinal relaxation times, $\mathrm{T}_{1}^{\mathrm{C}}$. The typical profile of relaxation indicates three components having short $(5.5 \mathrm{~s})$, intermediate $(10-130 \mathrm{~s})$ and long $(300-500 \mathrm{~s})$ characteristics $\mathrm{T}^{\mathrm{C}} \mathrm{s}$. In particular, in a drawn sample, the intermediate component always shows good chain orientation relative to the draw direction, and thus this component is a candidate for tie chains (orientation, mobility in excess of the crystalline chain mobility). Unfortunately, the third criterion used to identify tie chains in PET, namely, spatial proximity to the disordered regions, is a harder criterion to apply in polyethylene and attempts have not been successful in this regard. At present, therefore, the amount of intermediate component is being identified primarily with interfacial material. A melt- (or pressure-) crystallized isotropic sample is found to have 15 to 35 percent less intermediate component than a cold-drawn (at $100{ }^{\circ} \mathrm{C}$ ) material. Moreover, in the samples studied, a sample with a draw ratio of 10 has more intermediate component than one whose draw ratio is 20 . It is also found that the amount of long relaxing (crystalline) material underestimates crystallinity when compared with normal measures, i.e., DSC. At the same time, the sum of the long and intermediate components overestimates crystallinity. Furthermore, the high pressure crystaliized sample, which has lamellar widths of a few hundred nanometers, shows 15 percent intermediate and no short component. For this latter sample, which by density or DSC one would label as 95 percent crystalline, the 15 percent seems high. It, therefore, seems reasonable to assign the major portion of the intermediate component to regions near the fold surface of a crystal. It can be concluded that a significant contribution to the heat of melting arises from this region.

The time constants for the long relaxing (crystalline) components vary considerably. Unfortunately, the mechanism of this very slow relaxation is not fully understood. In the isotropic samples there seems to be a definite dependence on lamellar thickness, but comparable lamellar thickness in drawn versus isotropic samples do not yield comparably long $T_{1}^{C}$. An interpretation of this difference is that cold drawing produces crystallites which contain more crystal defects than in melt crystallization. Whether this ultimately will affect creep is possible, but not known.

\section{Quantitative Determination of the Monoclinic Crystalline Phase in Polyethylene}

Under certain circumstances of mechanical deformation, the monoclinic crystalline phase appears in polyethylene. This phase can be detected easily by $x$-ray wide angle techniques, however, the quantitative determination of the relative amounts of monoclinic and the usual orthorhombic crystalline forms in polyethylene is a more difficult task using $x-r a y$ techniques. Solid state ${ }^{13} C$ NMR provides a way of distinguishing these two crystalline phases. Moreover, it has been demonstrated in a ultrahigh molecular weight sample that the ratio of spectral intensities is the true ratio of amounts of these phases present. It has also been argued that the NMR technique, when reasonable care is taken, will give very good quantitative results, no matter what the history of the sample preparation is.

\section{Investigation of Ceilulosic Materials Used in Papermaking}

This work was partly sponsored by the Bureau of Engraving and Printing (BEP). The remaining work was undertaken in collaboration with Professor Rajai-Atalla of the Institute of Paper Chemistry.

The work sponsored by BEP was a continuation of efforts to examine changes in cellulose-water interactions and changes in microstructure which occur during mechanical beating of pulps used in papermaking. In addition, any comparisons of different raw materials, notably cotton and wood cellulose, are important oecause BEP is looking for cheaper substitutes for currency paper which now utilize primarily cotton and linen celluloses.

Previous ${ }^{13} \mathrm{C}$ NMR studies of cotton and wood pulp had pointed to the conclusion that wet beating did not change the microstructure of the elementary fibril, i.e., there was no significant increase in surface area which could be attributed to wet beating. Certain possible distortions of the NMR spectra, which could reflect on the validity of these conclusions, were recognized at that time and part of this year's effort was to investigate whether these spectral distortions were significant. 
The results of those experiments suggest that no significant intensity distortion occurs between the spectra of the wet and wet beaten materials, be they cotton or wood pulp. However, relaxation time evidence suggests that the wood pulp has picked up paramagnetic impurities which shorten its relaxation times so that the relative signal strength of the surface resonances compared to crystaliine interior resonances is smaller by the order of 10 percent in the wood pulp relative to the cotton. Thus, whereas some distortions exist which increase the relative intensities of the interior crystalline resonances compared with the remaining resonances, the surface area changes due to wet beating in any of these materials, are correctly monitored and are undetectable.

With regard to the interaction of cellulose and water, one of the interesting results is the similarity (within 15 percent) of magnetization transfer rates between water and the cellulose matrix in the wood pulp compared with the cotton. In the magnetization transfer experiments, proton spin magnetization is prepared to be non-zero only in the water as an initial condition. Then, due to proton chemical exchange, or dipolar spin exchange, some of the magnetization from the water moves into the cellulose. In the wet and wet beaten samples, the amount of water protons is well in excess of the number of cellulose protons, so magnetization transfer occurs in the presence of a largely constant water magnetization. The rate of transfer, normalized to the amount of cellulose present, might then be approximately related to surface area. The wood pulp was found to have a transfer rate in both the wet and wet beaten preparations about 15 percent higher than that in the cotton preparation. If these rates reflect relative surface areas, this is a surprising result, farticularly from the point of view that the non-freezing water in the wood pulp was measured to be SO percent higher than that of cotton. This would suggest that on average, some non-freezing water is not in contact with the surface, or perhaps that the few percent residual lignin in the wood pulp modifies the water/cellulose interaction in such a way that even though water does not freeze, magnetization transfer rates are partially inhibited.

The work carried out jointly with Professor Atalla focussed on the use of magic-angle spinning techniques for obtaining ${ }^{13} \mathrm{C}$ spectra in order to investigate heterogeneity of crystal structure in native celluloses. The interesting result of this work is that native cellulose appears to be a crystalline composite of two different forms. The exact nature of the differences is not defined by the NMR. The possibility that this heterogeneity may also be reflected as heterogeneity in mechanical properties or in water-cellulose interactions could have significant consequences in dictating end-uses of these various native celluloses.

\section{The Morphology of Crystalline Polymers}

F. A. Khoury, L. H. Bolz, and J. Mazur

Investigations on the morphology of crystalline polymers during the past year have been mainly in the areas outlined below.

\section{The Effect of Uniaxial Extension on the Fine Structure of Ultra High Molecular Weight Polyethylene (UHMWPE)}

This work is sponsored by the Bureau of Medical Devices of the Food and Drug Administration. It is part of a study of the relation between the fine structure and the mechanical properties af UHMWPE. This polymer is used in the manufacture of several types of orthopedic protheses.

The deformation of compression molded specimens of UHMWPE ( $M$. Wt. $2 \times 10^{6}-4 \times 10^{6}$ ) when they are stretched uniaxially is characterized by the following features [1,2]: (1) In contrast with lower molecular weight polyethylenes (e.g., M.Wt. $1 \times 10^{5}$ ) the specimens do not exhibit any gross necking. (2) The stretched specimens contract substantially when they are released under zero tension. For example, specimens made from polymer of M.Wt $2 \times 10^{6}$ which were stretched to strains of 0.25 and 3.0 at room temperature had residual strains of 0.1 and 1.9 respectively 24 hours after they were released. (3) For initial strains of up to 3, deformation does not occur uniformly throughout the specimens. This feature is associated with the retention of a memory of the "grain-like" character of the raw polymer in the molded samples, even though the polymer is heated to $60{ }^{\circ} \mathrm{C}$ above its melting point during the molding process. Evidence derived from light and scanning electron microscopical studies indicates that regions corresponding to the boundaries between the original raw polymer "grains" deform preferentially. (4) The weight percent crystallinity of unstretched specimens (which varies slightly with molecular weight) is close to 60 percent, and the unit cell structure in the crystalline regions is the commoniy observed orthorhombic structure. The majority of the crystallites in deformed specimens (stretched/released) having residual strains in the range 
0.1 to 2.9 are also orthorhombic. These specimens also contain a small fraction of the metastable monoclinic crystalline phase of polyethylene.

Current investigations are aimed at determining the fine structural changes which occur during the initial stretching of specimens to various strains, and upon subsequent contraction when the tension is released. The manifestation of polymorphism (i.e., the occurrence of the monoclinic crystalline phase in addition to the orthorhombic phase) and the progressive changes in orientation of orthorhombic and monoclinic phases during stretching up to a strain of 1.0 has been examined using wide angle $x$-ray diffraction.

The following are among the features observed: (a) The lowest strain at which the occurrence of the monoclinic phase was observed was 0.05 . (b) At a strain of 1.0 the distributions in the angle of inclination of the chain axis in the orthorhombic and monoclinic crystallites relative to the stretching direction are peaked at inclinations of about $36^{\circ}$ and $30^{\circ}$, respectively. (c) $A$ substantial decrease in the content of monoclinic phase occurs in all the samples stretched to strains in the range 0.05 to 1.0 when they are released and allowed to contract under zero stress. (This has also been found to be the case in specimens stretched to a strain of 3.0 ).

Small angle x-ray diffraction and electron microscopy are being applied to examine the changes in morphology (texture) associated with the changes in crystalline orientation and the manifestation of. polymorphism mentioned above. The application of ${ }^{13} \mathrm{C}$. NMR as a method for determining quantitatively the relative amounts of the orthohombic and monoclinic phases in UHMWPE is described elsewhere in this report.

\section{The Morphology of Polyethylene Crystals Grown From Solution}

A study of the effects of crystallization temperature and molecular weight on the lateral growth habits and the aspect ratio $r$ ( $r=$ ratio of the dimensions of the lamellae parallel to the [010] and [100] directions) in polyethylene crystals grown from 0.01 percent solutions in heptyl acetate and dodecanol at temperatures in the range $95^{\circ} \mathrm{C}$ to $120^{\circ} \mathrm{C}$ has been completed. Scanning transmission electron microscopy coupled with electron diffraction are currently being used to determine the nature of the non-planar conformation of the crystals whose aspect ratios vary between 1.6 to 6 depending on solvent, crystallization temperature and polymer molecular weight. Interest in these crystals stems from their usefulness as models (admittedly idealized ones) for the constituent lamellae in polyethylene spherulites grown from the molten state [3]. One of the objectives of our current work is to determine whether the relationship between crystals having a particular aspect ratio and the corresponding crystal conformation is unique or whether it varies with the molecular weight of the polymer.

\section{The Energies of (110) and (200) Folds in Polyethylene Crystals}

The energies of (110) and (200) folds in polyethylene crystals are of interest in the elucidation of the origins of two features of the morphology of solution-grown crystals and that of the constituent lamellae in melt grown spherulites namely: (1) The occurrence of fold staggering resulting in the formation of hollow pyramidal, tent-like or otherwise non-planar lamellae, and (2) the dependence of the lateral growth habits of the lamellae on the crystallization conditions. The feature of interest in the latter context is the dependence of the aspect ratio $r$ ( $r=$ ratio of the dimensions of the lamellae parallel to the [010] and [100] directions) in the crystals on the crystallization conditions. The ratio $r$ is governed by the ratio of the growth rates normal to the (110) and (200) faces. The higher that ratio the higher $r$.

As an initial step in examining the possible relationships between the above mentioned features and the (110) and (200) fold energies, minimum energy calculations for models of such folds were previously carried out for the following cases assuming adjacent reentry: (a) (110) folds surrounded by and interacting with kindred folds situated in the same and in adjoining (110) planes, lamella surface (001); (b) (200) folds surrounded by kindred folds situated in the same and adjoining (200) planes, lamella surface (001); (c) (200) folds surrounded by kindred folds packed in a staggered manner, i.e., lamella surface (201).

Calculations have now been completed for three models of staggered (110) folds, namely for the cases in which the lamella surface is (112), (111), and (312). The results expressed in kcal/mole of fold are summarized in Table I in which we confine ourselves to giving data for the energetically most favorable among the various models we have considered for each type of unstaggered or staggered folds containing 7 methylene groups in the fold. 
As can be seen from this table staggering results in a lowering of the energy of (200) folds. This is not the case however in the (110) folds. Since, for example, the folds in the distinct constituent $(200)$ and (110) sectors in polyethylene crystals grown from xylene are staggered, this latter result is unexpected and remains unexplained at present.

Comparison of the energies of (110) and (200) folds is also of interest in connection with the known effects of crystallization temperature on the lateral growth habits of polyethylene crystals, for the following reason.

When the crystallization temperature is increased, the aspect ratio $r$ of the crystals becomes larger, i.e., the ratio of the growth rates normal to (110) and (200) faces increases with crystallization temperature. An analysis [4] of the variation of $r$ with temperature for crystals grown from solution in xylene in terms of the kinetic theory of polymer crystal growth, has led to the result that the ratio of the end (fold) surface free energies, $\sigma^{200} / \sigma^{110}$, associated with (200) and (110) folds should be greater than unity. $\sigma^{20} / \sigma^{11}$ was found to be between 1.1 and 1.3 depending on the growth regime assumed. The models of staggered folds which correspond most closely to those in the crystals whose growth habits were used as a basis for the above-mentioned analysis are the $[(110),(312)$ surface] and the $[(200),(201)$ surface] models. The results of the fold energy calculations for those folds would have been consistent with the results of the analysis of the crystal habits had the energy of the latter type of (200) fold been greater than that of the [(110), (312) surface]. As can be seen from Table I, this is not the case. The calculated energies for these two types of folds are very nearly equal. The energy of the (110) fold is in fact $\sim 0.2$ $\mathrm{kcal} / \mathrm{mole}$ higher than the (200). A possible explanation of the apparent discrepancy between the results of the fold energy calculations and the analysis of crystal habits is that (i) statistical variations from the regular type of stagger assumed in the fold energy calculations occur in the crystals and (ii) that these variations give rise to deviations from the calculations based on the ideal ized models.

[1] McKenna, G. B.; Khoury, F. A. ; Crissman, J. M. Nat. Bur. Stand. (U.S) NBSIR 81-2209; 1981.

[2] Crissman, J. A.; Khoury, F. A.; McKenna, G. B. Nat. Bur. Stand. (U.S.) NBSIR 82-2493; 1982.

[3] Khoury, F. A. Faraday Disc. Chem. Soc. 68: 404; 1979. [4] Passaglia, E.; Khoury, F. A. in press.

\section{The Necking of Semicrystalline Polymers in Tension}

\section{J. Zapas and J. M. Crissman}

In tensile tests on polymeric bars the initial motion of homogeneous uniaxial extension can change to inhomogeneous motion called "necking." The occurrence of a neck is influenced by environmental factors such as temperature, pressure, and ambient atmosphere, as well as material related properties such as molecular weight, molecular weight distribution, and specimen preparation history which may be important in determining the degree of crystallinity. Although the phenomenon of necking is readily observed in most semicrystalline polymers, it can also occur under certain conditions in glassy polymers. Until quite recently most of the published work was concerned with studies of the necking process in polymers in which the deformations involved were accomplished with a hard machine where the specimens were extended at constant rate of clamp separation.

More recently, Zapas and Crissman [1-3] reported the results of uniaxial creep experiments under constant load for different polyethylenes under varied loads and temperatures. Several of these experiments lasted for months before necking was observed. A graphical procedure was proposed for estimating the time $t_{c}$ of first departure from the initial spatial homogeneous motion induced in a tensile creep specimen; $t_{c}$ often preceded by a constant factor the time at which the neck became easily visible. Zapas and Crissman considered that necking in polymers occurred due to an instability arising from the non-linear viscoelastic behavior of the specimen. 
This work was followed by a theory presented by Bernstein and Zapas [4] who extended the work of Ericksen [5] on the discontinuous deformations of solid elastic bars to viscoelastic materials which obey the Bernstein, Kearsley, and Zapas (BKZ) theory [6]. As in the case of Ericksen's work, phenomenon of necking, but it does cannot predict a priori when the material will exhibit the on the stretch history.

In order to examine the model proposed by Bernstein and Zapas, a rather detailed investigation has been made on a quenched isotactic polypropylene under a wide variety of stress and strain histories in uniaxial extension. These include single step stress relaxation, constant rate of measurements were made up to and beyond the creep under a fixed applied load. In each case the the available data one can construct is possible to predict the point at which necking will of extrapolating the available data into regions which are not occur for other strain histories. By shown that, for a material between the instability theory of Bernstein and

\section{References}

[1] Zapas, L. J.; Crissman, J. M. Poly. Eng. \& Sci. 19(2): 104; 1979.

[2] Crissman, J. M. ; Zapas, L. J. Poly. Eng. \& Sci. 19(2): 99; 1979.

[3] Zapas, L. J.; Crissman, J. M. Durability of macromolecular materials. R. K. Eby, Ed. ACS Symp. Series, No. 95: 301; 1978.

[4] Bernstein, B. ; Zapas, L. J. J. Rheo1. 25: 83; 1981.

[5] Ericksen, J. L. Elasticity 5: 191; 1975. [6] Bernstein, B.; Kears ley, E. A. ; Zapas, L. J. Trans. Sco. Rheol.
7: 391; 1963.

\section{A Study of the Morphology and Mechanical Properties, and Their Dependence on Thermal History and Processing of UTtra High Molecular Weight Poly-}

\section{J. M. Crissman}

Ultra high molecular weight polyethyiene (UHPWPE) is used in the manufacture of a variety of orthopedic joint prostheses. One factor which limits the lifetime of a prosthes is is the durability of the UHMWP component. The length of time between device implantation and failure depends upon time dependent processes which result from the clinical loading environment. In clinical use, associated with wear, and even fracture. Both the occur as a result of creep, wear, fatigue determined by the polymer chemistry and morpholoth the mechanical properties and durability are orientation, spherulite size and texture distribution influence mechanical performance. Under cos molecular weight and molecular weight Food and Drug Administration, we are in the fourth year of mechanical behavior of UHMWPE. A summary of earlier york of a study concerned with the morphology and Polymer Science and Standards Division 1981 and 1982 Annual Reports. Aspects of the morphology of UHMWPE which have been studied during the past year are summarized
elsewhere in this report.

The mechanical properties studies were continued and expanded to include the creep and recovery behavior, in both extension and compression, at elevated temperatures. In previous work described behavior of UHMWPE in 1982 Annual Report, it was reported that the room temperature short time creep and recovery the form,

$$
\phi\left(\sigma, t_{1}\right)=(\text { const }) g(\sigma) t_{1}^{1 / 3}
$$

where $g(\sigma)$ is a function of stress alone and $t_{1}$ is the duration of the creep step. More recent temperature is increased. 
Another important area of study, and one which relates to the performance of the polymeric component of surgical implants, is the influence of $\gamma$-irradiation on the mechanical properties of UHMWPE. For purposes of sterilization, the UHMWPE components of prostheses are routinely subjected to relatively low levels of $\gamma$-irradiation ( 2 to $4 \mathrm{Mrad}$ ). $\gamma$-irradiation can produce both crosslinks and chain scissions. Whether or not a particular property is enhanced or diminished will depend upon which of the mechanisms is dominant. In normal lower molecular weight polyethylenes it is known that radiation increases the tensile modulus and yield stress whilst reducing creep. The creep and recovery behavior of $\gamma$-irradiated UHMWP is being investigated in uniaxial extension for specimens having radiation dosages in the range 1 to $6 \mathrm{Mrad}$. It is found that for applied stresses in the range expected in clinical use (2 to $4 \mathrm{MPa}$ ) a dose of 3 Mrad is sufficient to noticeably reduce the amount of creep. On the other hand, it is also found that does of from 3 to 6 Mrad are sufficient to significantly reduce the environmental stress-crack resistance of UHMWPE from the value in air.

\section{Information Handbook on Polymeric Materials Used in Orthopedic Devices}

\section{J. M. Crissman and G. B. McKenna}

A project is underway to write a materials handbook on the principal polymeric materiais used in surgical implants. This work is being done for the FDA, Bureau of Medical Devices, as part of task 80-01 NBS-BMD Interagency Agreement. Included in the handbook will be sections on raw materials characterization, processing, and characterization of morphology and mechanical properties of fabricated forms of polymer. The intended audience for the handbook includes the FUA/BMD staff, resin manufacturers, fabricators, medical researchers, and surgeons.

\section{Instabilities in the Flow of Viscoelastic Fluids}

\section{R. W. Penn and L. J. Zapas}

The rates of production of many polymer processing operations are limited by the onset of flow instabilities. Such operations include filament extension processes (the instability is referred to as "draw resonance") and die extrusion processes (the instability is generally termed "melt fracture"). Bernstein and Zapas have shown how such instabilities can be related to the time dependent, constitutive behavior of non-linear viscoelastic materials [I]. Their analysis involves the behavior of the material in both a "hard device" where the strain is imposed and one observes the stress and in a "soft device" where the stress is imposed and one observes the strain. In a typical hard device experiment one subjects a specimen to a constant rate of strain at zero time and measures the stress. In the range of high strain rate approaching unstable flow the stress increases to a maximum and then decays to a steady state value [2]. This behavior was adequately described by the BKZ fluid [3] but in light of discrepancies between BKZ behavior and the behavior of these fluids in other strain histories, it becomes of interest to study them in a soft machine and to obtain a better description of their behavior.

In the current work a Rheometrics Mechanical Spectrometer has been modified so that it can be operated in torque control. This required the addition of a more stable torque transducer. The output of this transducer was sent to the "external" input on the position control card and the position feed back signal was interrupted on that card. Outputs from the torque and position transducers were linearized and amplified to levels compatible with our digital data acquisition and control system. The servo-system was tested and optimized with standard Newtonian fluids. At constant stress these fluids show only a constant strain rate and zero recovery on removing the stress.

Shear creep and recovery measurements were obtained in cone and plate geometry on a 19.3 percent solution of polyisobutylene $L-100$ in cetane at various shear stresses approaching the range of flow instability. At these high values of stress, the rate of shear ceases to be a monotonic function of time. It starts off at a large value on application of the step in stress. It then goes through a minimum before approaching a steady state value. Significant recovery is observed on reducing the stress to zero.

The creep and recovery cannot be correlated using non-linear Boltzman-like superposition. A one dimensional mathematical description is being developed which will correlate the creep and recovery data. It is a description of a fluid and is properly time invariant. The strain is 
expressed as the sum of two integrals. The kernal of the first is a non-linear function of the value of the instantaneous stress. The second is a non-linear functional of the history of the rate of shear.

[1] Bernstein, B.; Zapas, L. J. J. Rheol. 25(1): 83; 1981.

[2] Zapas, L. J.; Phillips. J. C. J. Rheol. 25(4): 405; 1981.

[3] Bernstein, B.; Kearsley, E. A.; Zapas, L. J. Trans. Soc. Rheol. 7: $391 ; 1963$.

\section{The Distribution of Stresses in Crazes at the. Tips of Uniformly} Extending Cracks

\section{E. Passaglia}

The work reported in the last report (Polymer Science and Standards Division 1982 Annual Report) has been brought to a conclusion. It was shown there that in a craze at the tip of a uniformly moving crack, the stress at a point $\beta$ in the craze is a function of the derivative of the displacement at that point, under the assumption that the craze thickens by a drawing process. The stress is thus identified as the drawing stress. Specifically, if $\ell$ is the displacement, and $\beta$ and $\eta$ are normalized coordinates within the craze, then

$$
\frac{\partial w(\beta)}{\partial \beta}=\frac{C_{e}}{2 \pi} \quad \frac{1}{0} \int \sigma(\eta)(\beta / \eta)^{1 / 2} d \eta / \beta-\eta
$$

where $C_{e}$ is the plane strain compliance. Since the stress is a function of the displacement derivative, which is equivalent to the draw rate, an integral equation for the stress is obtained in the following manner.

Over fairly wide ranges of draw rates, the drawing stress of many polymers. is linear in the logarithm of the draw rate [1], i.e.,

$$
\sigma_{D}=\sigma_{r}+\sigma_{s} \log \varepsilon / \varepsilon_{r}
$$

where $\varepsilon$ is the draw rate, $\sigma_{r}$ is the drawing stress at the draw rate $\varepsilon_{r}$, and $\sigma_{s}$ is the rate coefficient. Denoting the integral in Eq. (1) by I, we obtain as the equation to be solved

$$
\sigma(\beta)=\sigma_{r}+\sigma_{s} \log \frac{I(\beta)}{I(0.5)}
$$

where the reference draw rate is arbitrarily chosen to be at the mid point of the craze.

A computer algorithm has been developed to solve this equation. The following assumptions are entered into the solution.

(1) The value of the stress at the craze tip is kept constant to represent the yield stresses.

(2) The value of the stress at the mid point of the craze is treated as a parameter, and solutions for various values obtained, i.e., for $\sigma(0.5) / \sigma(0)$ from 0.5 to unity.

(3) The value of the rate coefficient is also treated as a parameter and solutions have been obtained for $\sigma_{s} / \sigma_{r}$ ranging from 0.03 to 0.3 , which covers the range for most polymers.

The stress distributions obtained all show a peak at the craze tip and one at the crack tip, in agreement with the observations of Kramer [2]. The heights of the peaks are determined by both the chosen stress at the mid point and the value of the rate coefficient, with the latter largeiy determining the height of the peak at the crack tip.

[I] Ward, I. M. Mechanical properties of solid polymers. John Wiley N.Y., 1971.

[2] Kramer, E. J. Advances in Polymer Sci. 50: 1983. 
Physical Ageing in Polymeric Glasses Subjected to Large Deformations

G. B. McKenna and A. J. Kovacs ${ }^{1}$

${ }^{1}$ Centre de Recherches sur les Macromolecules (CRM), 67083 Strasbourg, France

Upon isobaric cooling through their glass transition range undercooled liquids, inciuding rubbery polymers, are known to depart from their thermodynamic equilibrium [1]. Non-equilibrium glassy structures are intrinsically unstable, since they spontaneously evolve towards their equilibrium structure which depends on temperature alone [2]. This approach towards structurai equilibrium is clearly reflected by the time dependence of the volume and enthalpy of the glass as well as its mechanical (viscoelastic) properties.

The purpose of the present work is to investigate the ageing behavior of polymethylmethacrylate (PMMA) as a preliminary to a larger program to measure dilatometrically the volume changes of polymer glasses subjected to increasing torsional deformation after various thermal treatments. Such an attempt should lead, in fact, to some important conclusions (both from practical and fundamental viewpoints) about the viscoelastic behavior of glasses at moderate and large deformations as a function of their thermal and mechanical histories.

The results obtained on the ageing behavior of PMMA reveal a few, though significant, discrepancies as compared to the general picture emerging from previous investigations $[3,4]$. In the linear viscoelastic range, the relaxation isotherms, measured at increasing ageing times, cannot be superposed by mere. shifting along the time axis below some critical temperature range $\left(60^{\circ}<T_{c}\right.$ $<80^{\circ} \mathrm{C}$ ). This critical temperature range decreases, however, as the deformation increases. Such effects may be accounted for by a change with increasing ageing time in the shape of the relaxation spectrum at short times [5].

Furthermore, the shift rate for the torque relaxation function decreases much more rapidly with increasing deformation than does that of the normal force relaxation function. This difference in shift rates for the torque and normal force responses increases with decreasing temperature, and implies that the molecular processes controlling the torque (shear) response are less sensitive to changes in structure (free volume) during ageing than are those determining the normal force response. A complete understanding of these phenomena requires simultaneous (as opposed to parallel) measures of the changes in volume, torque and normal force induced by a given deformation under systematically varied thermal histories. Such.investigations are currently underway at CRM in Strasbourg using the apparatus described in last year's Polymer Science and Standards Division annual report $[6]$.

[1] Simon, F. Erg. Exakt. Naturwiss. 9: 222; 1930.

[2] Kovacs, A. J. Fortsch. Hochpolym. Forsch. 3: 394; 1963.

[3] Struik, L. C. E. Physical aging in amorphous polymers and other materials. Elsevier, Amsterdam, 1978.

[4] Chapter 8 in ref. 4.

[5] Kovacs, A. J.; Stratton, R. A.; Ferry, J. D. J. Chem. Phys. 67: 152; 1963.

[6] Polymer science and standards division annual report. Nat. Bur. Stand. (U.S.) NBSIR 82-2607, March 1983.

The Effect of Elastic and Plastic Deformation on the Solubility of Ethyl Acetate Vapor in Polyvinylidene Fluoride $\left(\mathrm{PVF}_{2}\right)$

J. C. Phillips and A. Peterlin

The transport of liquids into semicrystalline polymers has been known to be influenced by the strain deformation of the polymeric material. Often, the diffusivity and solubility of gases and vapors in the strained material are also dramatically reduced from those of the unstrained material. The extent of the reduction of these transport properties depends on the strain level $\varepsilon$, the vapor pressure $p$, and the elongation time $t_{h}$. According to the fractional free volume theory (FFV), the solubility $S=c / p$ ( $c=$ equilibrium concentration; $p=$ pressure) of the permeant is proportional to the elastic deformation. 
Strain recovery measurements were performed in air at $24^{\circ} \mathrm{C}$ and in an IR cell at $30{ }^{\circ} \mathrm{C}$ as a function of ethyl acetate vapor pressure. The experiments in air were done by elongating the film on a stretching irame, holding the film at this elongation for a time $t_{h}$ and then releasing the film. The decrease in length was followed as a function of time with a cathetometer.

In the IR cell the experiments in the vapor environment were performed by first sorbing the vapor to saturation and then straining the sample. The sample was held at this strain for a time $t_{h}$ and the stress was manually nulled to obtain strain recovery as a function of time. From the strain recovery/time curve, the elastic and plastic components of the total strain were obtained by extrapolation of the curves to $t=0$.

The IR cell was also used to obtain solubility data as a function of strain and pressure. The experimental procedure consisted of stretching the sample and admitting the vapor to the sample chamber of the IR cell at some time $t_{0}$ after stretching. The carbonyl band of liquid ethyl acetate was monitored as a function of time to determine the amount of the liquid adsorbed by the polymer film. From the sorption curve, the equilibrium solubility, $S$, was determined from the IR equilibrium absorbance. The results indicate that the FFV concept applies reasonably well to the $\mathrm{PVF}_{2}$ /ethyl acetate system under uniaxial deformation. 
The great emphasis on light-weight, energy-efficient materials is producing a rapid growth of composites as a high payoff application of polymers. The addition of a reinforcement, either particles or fibers, to polymers can dramatically improve their performance, and as a result they. can be utilized in a wide range of generic applications where the requirements are beyond the capabilities of simple polymers. This expanded potential has led to increased usage in diverse areas ranging from transportation to construction. In addition to their light-weight, major factors behind the expanded applications of composites include versatility in fabrication, high corrosion and fatigue resistance, and the potential to significantly reduce the need for critical materials such as cobalt and chromium whose limited supply is of critical concern to the national interest.

Although composite materiais have great potential, the same multicomponent composition that is responsible for their high performance also gives rise to numerous problems with regard to processing and reliability. Since the composite field is still at an early stage of development, many of these problems can be directly attributed to a lack of basic knowledge and adequate test methods. In response to this need, a new Task was initiated in 1982 . The objectives were to carry out long range research to provide industry and other Government agencies with test methods, materials property data and failure prediction models that are essential if the full potential of these materials is to be achieved. In line with the two primary problem areas mentioned above, two major thrusts were developed and these continue to be the basis for the composites effort. The first seeks to gain a better understanding of the basic science involved in processing and fabrication of these materials while the second addresses the development of the basic science concepts, methods, and data needed to assist in improving durability and enhancing the capability for failure predictions.

Task programs have been developed to address both areas of concern. A number of projects are studying cure, i.e., the chemical reactions that occur during processing and represent the most important factor in successful fabrication. The work combines a variety of nondestructive measurement techniques so that a thorough understanding of the processes involved can be developed. The potential for employing one or more of these techniques for real time on-line control of cure is also being addressed. The second major thrust area concerns the failure behavior of both particle and fiber reinforced polymers. Special emphasis is placed on the development of techniques, such as $x$-ray scattering, which can provide information about the state of the material before and during failure. The programs seek to understand the basic failure mechanisms in polymers and how these mechanisms are transferred into composites.

To assist in conducting this broadly based effort, the task is utilizing a number of cooperative programs as well as the services of experts who spent time working in the Division during the last year. The cooperative efforts involve programs with NASA Langley Research Center, the Department of the Treasury, and the industrial research laboratories at Hercules and Hexcel. In addition, the joint research program with Dr. A. J. Kinloch of the Waltham Abbey Research Laboratory of the Ministry of Defense in England on the failure behavior of particle reinforced composites is continuing. Finally, foreign guest workers from Israel, Mrs. H. Weisshaus, and Taiwan, Mr. C. Yu, have actively participated in these Task programs during the last year.

\section{Interlaminar Fracture of Composites}

\section{L. Hunston, G. W. Bullman, and W. D. Bascom²}

${ }^{1}$ Hercules, Inc., Graphite Fiber Aerospace Division, Magna, UT

A major area of concern in the use of continuous-fiber reinforced composites is delamination, that is, the growth of cracks between the layers of fibers. Delamination can substantially reduce the performance of composites in numerous ways but compression strength is particularly sensitive to this type of damage. The conventional polymeric matrix resins, i.e., the material that binds the fiber together, are very brittle and therefore subject to damage particularly when loaded by impact. This damage involves matrix cracks which can easily grow into large areas of delamination. The problems this can generate in practical applications have led to a widespread interest in developing polymeric matrix materials with improved resistance to both initial damage and subsequent growth of this damage into delamination. In addition, there is a need to develop design data that can be used to predict the growth rate of delamination. Unfortunately, although a number of test methods are now being used, few if any have been developed to the extent that they can provide the quantitative information that is needed. 
In response to this problem, a joint program has been established among researchers at NBS, NASA Langley, the University of Illinois, and several composites manufacturers. As part of this program, NBS is examining the double cantilever beam test method for determining interlaminar fracture. The tests employ specimens with either rectangular or triangular geometries. A precrack is generated between fiber layers in the specimen and an increasing load is applied perpendicular to the precrack so that subsequent crack growth occurs between the layers of fibers. The critical value at which this crack growth begins is noted and converted to a fracture energy using appropriate equations.

During the past year, the effects of sample lay-up pattern on interlaminar fracture behavior have been studied and guidelines for specimen design have been formulated (1). In addition, preliminary experiments were performed to determine the effects of loading rate and temperature on interlaminar fracture behavior. With brittle matrix resins both the resin itself and the composite exhibit only small variations with changes in these variables over the ranges that are of interest. Tough resins, on the other hand, have a significant rate and temperature dependent fracture energy. The decrease in fracture energy with decreasing temperature and increasing cross-head speed is particularly important because a major concern with composites is damage resulting from impact loads. As a result, tests were conducted on selected samples of woven fabric reinforced composites using the double cantilever beam. The results are shown in Table I. These data indicate that, unlike the resin itself, the interlaminar fracture energy does not decrease with decreasing temperature and increasing cross-head speed over this range of conditions. In fact, if a trend exists, it's in the opposite direction. These preliminary results are encouraging but much more work needs to be done before the effects of temperature and loading history can be understood and predicted. Work currently in progress is examining these questions and extending the study to unidirectional composite specimens.

[1] D. L. Munston and W. D. Bascom, Composite Technology Rev., submitted.

Table I: Fracture Energy $\left(\mathrm{kJ} / \mathrm{m}^{2}\right)$

Temperature

${ }^{\circ} \mathrm{C}$

Fracture Energy at Cross-Head

$0.008 \quad$ Speed $(\mathrm{mm} / \mathrm{s})$

0.8

\begin{tabular}{rrrr}
\hline 40 & 1.7 & 1.8 & 1.8 \\
24 & 1.7 & 1.7 & 1.9 \\
0 & 1.7 & 1.9 & 2.0 \\
-25 & 1.9 & 2.1 & 2.4 \\
\hline
\end{tabular}

Failure Behavior of Particle Reinforced Polymer Composites

D. L. Hunston, A. J. Kinloch ${ }^{1}$, and S. S. Wang ${ }^{2}$

1Ministry of Defense, PERME, Waltham Abbey, Essex, England

2Dept. of Theoretical and Applied Mechanics, University of Illinois, Urbana, IL

Most of the polymers used in stuctural application are inherently brittle and must be reinforced or toughened. One empirically developed method that has proven to be quite successful is the incorporation of small particles of a softer material in a matrix of the brittle polymer. Although composite materials of this type often have very high fracture energies their failure behavior is usually quite complex and involves large viscoelastic effects.

In an effort to gain a fundamental understanding of the failure behavior of such materials, NBS is currently studying two different model systems: a polysulfone filled with polytetrafluoroethylene particles and an epoxy filled with epoxy/polybutadiene particles. The first system is of interest to the Navy for certain sealant applications while the second serves as a model for most commercial structural adhesives. The program focusses on developing test methods for such materials and studying the mechanisms of failure. This is a joint program involving scientists from the Ministry of Defense (Waltham Abbey Laboratory) in England and the University of Illinois. 
During the past year an extensive study was performed to examine the viscoelastic fracture behavior of an rubber-modified epoxy. Based on these results a mechanism for toughening was formulated. This mechanism involves both dilatation via cavitation in the rubber particles or at the particle-matrix interface and shear plastic flow of the epoxy matrix. This mechanism explains all of the observed fractographic features over a wide range of test temperatures and cross-head speeds [1]. A two parameter failure criterion was then proposed and this criterion fits all of the failure data over the complete range of conditions studied [2].

The investigation also studied the failure behavior of these materials in adhesive bonds. The well known dependence of fracture energy on bond thickness was examined and a qualitative explanation was formulated based on an interaction between the size of the crack tip deformation zone and the bond thickness. By using this picture, the optimal band thickness could be predicted with a time/temperature dependent. elasticplastic model [3]. The quantitative form of the bond thickness dependence, however, could not be predicted and thus even a modified fracture energy approach has not provided a unique failure criterion. By using a nonlinear elastic-plastic constitutive equation in a finite element analysis, an effort was made to use a " $J$ " integral approach to address this problem. The results suggest that even this more general approach does not provide a unique criterion for failure. Consequently, more fundamental studies are required before the failure behavior of rubber-modified epoxy adhesives can be understood and predicted.

[1] Kinloch, A. J.; Shaw, S. J.; Tod, D. A. ; Hunston, D. L. Polymer; in press.

[2] Kinloch, A. J.; Shaw, S. J.; Hunston, D. L. Polymer; in press.

[3] Hunston, D. L.; Kinloch, A. J.; Shaw, S. J.; Wang, S. S. Proc. Intl. Symp. on adhesive joints; in press.

Cure Monitoring in Polymers and Composites via Nondestructive Techniques

D. L. Hunston, B. M. Fanconi, F. I. Mopsik, C. C. Yul ${ }^{1}$ and H. Weisshaus ${ }^{2}$

${ }^{1}$ Chun-Shan Inst. of Science \& Technology, Taiwan

${ }^{2}$ Armament Development Authority, Haifa, Israel

The rapidly expanding use of polymers in structural applications has generated much interest in the new field of polymer processing with chemically reacting (i.e., polymerizing) materials. The commercial importance of these polymer systems combined with the general lack of knowledge in this area has produced an urgent need to investigate the basic science of processing with reacting polymers and to develop nondestructive test methods to explore this area. These needs include both laboratory test techniques which permit the study and optimization of the processing reactions and on-line techniques that can be used to monitor and adjust processing variables.

A fundamental approach to this problem, however, requires a variety of measurement techniques since important events occur on the molecular level, the morphological level, and the mechanical properties level. The approach taken in the program currently underway in the Polymers Division is addressing this difficulty by combining techniques from three different areas: spectroscopy, dielectrometry, and mechanical properties characterization. The spectroscopic technique (Fourier transform infrared measurements) has the potential to examine curing (polymerization) reactions at a molecular level indicating what the reactions are and how rapidly they proceed. The mechanical characterization technique involves the ultrasonic test method developed previously in the NDE program, [1,2]. In this method an ultrasonic shear wave is propagated in a thin quartz strip that is coated with the sample to be tested. By measuring the wave in the quartz, information related to the mechanical properties of the coating can be obtained. Consequently, this method provides data directly relevant to processing and performance. The dielectric experiments, which utilize the unique NBS time domain dielectric spectrometer, provide information on mobilities at a morphological level, i.e., a size scale intermediate to the other two methods. By combining these three techniques, it will be possible to obtain a much more complete understanding of the transformations that occur during reactive processing of polymers.

During the past year a feasibility study was conducted to determine if all three of these techniques could be used to monitor cure in similar chemically reacting polymer systems. These tests confirmed that such measurements could be made and thus demonstrated that an indepth development of each technique as a cure monitoring tool would be advantageous. In previous years the ultrasonic technique has been shown to be a valuable cure monitoring device which has a unique capability to nondestructively follow changes that occurred during the curing of polymers in thin films or restricted layers. In addition to this the technique has the potential for quantitatively 
determining mechanical properties. Over the past year this latter capability has been implemented. Since no other technique can provide equivalent measurements the only way to validate this capability for highly viscoelastic materials is to compare the results from the ultrasonics test with extrapolated values obtained by applying time-temperature superposition to low frequency data from torsional oscillation experiments on a thermorheologically simple material. Such tests were conducted using a polyvinyl chloride plastisol and the results are shown in Table I. In light of the large extrapolations involved ( 5 decades), the agreement between the two sets of results is excellent.

\section{Table I}

\section{Method}

U1trasonic Tests

Estimate from Superposition
Shear Storage Modulus (MPa)

\author{
Shear Loss \\ Modulus (MPa)
}
0.70
0.78

The successfui development of this technique has led to a desire by a number of other groups to implement a similar device for addressing their particular problems. For example, NBS is currently constructing such a device for the Bureau of Engraving and Printing so they can use it to study drying of printing inks [2].

The Fourier transform infrared studies of thermally initiated curing of vinyl ester systems was reported previously. [3]. During the past year infrared studies have been extended to UV initiated cure systems. These systems are desirable as it is not necessary to mix components to initiate the cure reaction. Thus, time available for fabrication is not limited as it is for the two component systems that are presently in use.

Vinyl ester systems containing UV initiated accelerators were examined as a function of the initial UV exposure. Somewhat surprisingly, the subsequent degree of cure (post curing) behavior of the UV initiated systems was not substantially different from that of the thermally induced curing resins. What is measured by the FT-IR technique is the remaining unreacted double bonds. Based on our results mixing of the two component systems was reasonably complete and the substantial amount of unreacted groups cannot be attributed to a nonuniform distribution of initiators.

Subsequent work will focus on the relationship between the functionality of the reactive monomers, ge 1 point, curing temperature and glass transition temperature of the incompletely cured material. The glass transition temperature effectively determines the maximum useable temperature of the system. This temperature is given by the temperature of cure in low functionality systems. It is anticipated that increasing the functionality will lead to a higher use temperature for a given curing temperature.

Future work in this program will also focus on the development of the time domain dielectric spectrometer as a cure monitoring device. This instrument, which was developed at NBS, has the capability to measure the dielectric constant of a material over a very wide range of times (or frequencies) in a single experiment. Preliminary studies on the latter phases of cure have shown that this capability makes it possible to follow changes in molecular mobilities that would otherwise be very difficult to detect because they appear only in the very low frequency range. This type of information is particularly useful since the final degree of cure is often a critical parameter in processing. As a result further development of this technique is highly desirable and will be a major goal next year.

[1] Hunston, D. L.; Cure monitoring of thermosetting polymers by an ultrasonic technique, Review of Progress in Quantitative Nondesstructive Evaluation, 0. 0. Thompson, ed. p. 1711, Plenum Press, 1983.

[2] Hunston, D. L.; Relationship between mechanical properties and performance of inks as the basis for quality control techniques. Nat. Bur. Stand. (U.S.) NBSIR 83-2691, April 1983. [3] Annual Report of the Polymers Science and Standards Division, Nat. Bur. Stand. (U.S.) NBSIR 82-2607, March 1983. 


\section{L. Hunston and G. W. Buliman}

Many commercially ieportant coatings dry via cure reactions that are initiated by oxygen and polymerize through double bond reactions. To study these important systems, however, a means must be found to expose all of the sample to oxygen. Experiments conducted at NBS have developed a test method to accomplish this. In this technique, the sample is placed on a 2 roll mill. The thin film generated on the mill maximizes the exposure to oxygen while the mixing action between the 2 rolls colistantly renews the outer surface thus preventing the formation of an outer crust.

In one set of experiments, the mill was heated to $80{ }^{\circ} \mathrm{C}$, and periodically small amounts of the sample were removed and tested for mechanical properties. In this way, the rheological changes associated with the cure reactions could be followed. One particularly useful measurement was the steady flow shear viscosity. If this parameter is measured at a series of shear rates, the results can be analyzed in terms of an initiation time followed by an exponential growth in viscosity with time (linear relationship in a log-linear graph). The slope of the best fit straight line and the intersection between this line and a horizontal line at the zero time viscosity value can then be used to characterize the early phase of the cure reaction in these systems [1].

[1] Hunston, D. L.; Rushford, J. L. ; Newitt, W. R.; Vaudreui1, B. A. Chemorheology of Thermosetting Polymers, C. May, Ed., ACS Symp. Series; in press.

\section{Novel Exciner Fluorescence Method for In-Situ Monitoring of Composite Materials Manufacturing}

$$
\text { F. W. Wang, R. E. Lowry, and W. H. Grant . }
$$

We have developed a novel excimer fluorescence method that can be adopted for monitoring in situ and nondestructively the manufacture of components made of fiberglass-reinforced composite materials. The need to mitor and control the processing variables in the manufacture of these composite components becomes pressing as they become widely used in critical aerospace applications. The excimer fluorescence method monitors the resin viscosity that must be carefully controlled during processing if the rejection rates of these components are to be reduced.

The excimer fluorescence method determines the resin viscosity by measuring the fluorescence properties of a trace acount of an excimer-forming probe dissolved in the resin. Each of the chromophores attached to the probe molecule can fluoresce as an isolated excited chromophore or combined intramolecularly with another chromophore in the ground state to form an excimer that fluoresces at its characteristic wavelengths. Since the rate of excimer formation depends on the medium viscosity, this viscosity can be determined from the ratio of the fluorescence intensities of the excimer and the isolated chromophore, when the resin is continuously illuminated. This method was successfully used to sonitor the viscosity changes of samples of methyl methacrylate undergoing polymerization.

This excimer fluorescence method can even be used to determine the resin viscosities of inhomogeneous or irregularly-shaped samples. For example, it has been successfully used to monitor the viscosity change of a methyl methacrylate sample filled with fiberglass. This insensitivity to inhomogeneity or irregular geometry, is important for in situ monitoring of the curing of composite structures in the factory environment.

\section{Amalysis of Thermoset Resin Polymerization Kinetics}

\section{G. A. Senich, R. E. Florin, and J. C. Phillips}

Composite parts are produced from thermoset resins by molding or laminating operations which generally require either a long time in a closed mold or press at a high temperature and pressure or a lengthy post-cure outsije the mold, again at high temperature. These long times for thermal polymerization or curing, the order of one to several hours, restrict the rate of production of composite articles. Rapidfy curing thermoset matrix resins could greatly reduce processing time and permit a large increase in the volume of fabricated composite output. 
Radiation initiated polymerization is one method for obtaining the rapid cure of a thermoset composite matrix. The most common radiation sources used to induce curing reactions are electron beams (EB) and ultraviolet (UV) light. Since the energy imparted by UV radiation is much less than for $E B$, special excitable molecules referred to as photoinitiators must be added to insure that exposure to UV light yields the desired chemically reactive species. Additional methods of promoting polymerization by radiation include infrared (IR), microwave, and high frequency curing. It has been estimated that of all the radiation curing methods, IR has the broadest potential for use although, at present, EB and UV are exploited commercially to a much greater extent. Many industrial procedures utilize radiation and radiation curing, some for treating thin films and coatings and others for bulk articles. They include such diverse commercial processes as crosslinking of wire insulation, rubber tires, and dental fillings, preparation of resists for microelectronic device fabrication, setting coatings and printing inks, and sterilizing medical supplies.

The science and technology of curing organic materials with radiation is the subject of a review prepared as a background for further research [1]. Included are discussions of definitions of the curing process, motivations for exploiting curing with radiation, basic principles of the interaction of various forms of radiation with matter, and kinetic schemes for polymerization and oxidation, chain reactions which can be initiated by molecular species excited by the aforementioned interations. Various radiation sources, the equipment necessary to affect a radiation cure, and resin systems suitable for use with these sources are considered, and some practical problems unique to each radiation method are indicated.

The application of radiation curing to industrial operations is discussed, with particular emphasis given to processes which employ relatively thin films, such as inks and coatings.

An experimental program to measure the overall rate and determine reaction kinetics of thermoset curing reactions is in progress. The principle thermosetting systems of interest, epoxies, acrylated oligomers, and unsaturated polyesters/acrylics, are being initiated with UV and IR radiation as well as by more conventional thermal means. The effects of initiator structure, concentration, and solubility, reactant concentration, film thickness, reaction temperature, and radiant intensity are being studied. FTIR spectroscopy is being used to determine the extent of reaction of the participating chemical functional groups as curing progresses. Viscometric techniques at a variety of shear rates are employed to measure the increase in sample molecular weight while polymerization is taking place. Results from these experiments are being coordinated with the findings from photopolymerization measurements on identical materials by thermal analysis methods to provide a more complete explanation of the bulk polymerization kinetics of these rapidly curing thermoset resin systems. Novel methods for studying the kinetics of rapid curing, such as monitoring the mechanical property changes of a UV-irradiated film on a substrate by ultrasonic shear wave propagation, are also being employed. This program is providing a better understanding of the chemistry that occurs during processing of thermoset polymers and is aiding in the identification of materials for special applications, such as rapidly curing thermosets suitable for use as ink vehicles or repair formulations for the quick mending of composite parts.

[1] Senich, G. A.; R. E. Florin. Radiation curing of inks and coatings.

Nat. Bur. Stand. (U.S.) NBSIR 83-2722; June 1983.

Measurement of Photopolymerization of Polymers by Thermal Methods

J. H. Flynn

Considerable interest and activity has been exhibited in the past few years in polymerization reactions initiated by visible and ultraviolet light. Areas in which applications of photocuring are of interest include the ink, coatings, and dental fillings industries where techniques of rapid cure which avoid excessive heating and solvent removal are desirable.

Differential Scanning Calorimetry (DSC) is being used to monitor the kinetics of these photocure processes. This technique is particularly well suited for these investigations as the amplitude of a DSC trace is a robust measurement of the rate of cure and the area beneath the trace measures the enthalpy of reaction. 
The effects of light intensity, initiator concentration, temperature, specimen thickness, etc. upon the polymerization kinetics are being investigated for several industrially important resininitiator systems. A post irradiative "dark" reaction has been detected and it is also being investigated.

Variable induction periods and other anomalies becloud the initial phases of these reactions. Therefore, a technique of analys is has been adapted to these conditions which obtains kinetic parameters from derivative data in which the initial concentrations of reactants may remain unknown. By this method, the amplitude of the DSC trace, dq/dt, is represented by the equation $d q / d t=k\left(q_{0}-q\right)^{n}$, where $q$ is the enthalpy and $t$, the time. The rate constant, $k$, and reaction order, $n$, are determined from an appropriate function of $d q / d t$ vs. time, after an approximate value of $n$ has been estimated from tables in which ratios of times to reach fractions of completion are given for values of $n$. These analyses permit the determination of parameters for the relationship between rate and the factors influencing it, to be used in modeling mechanism.

The low thermal conductivity of polymeric materials is a chronic problem in the treatment of their kinetics as often heat flow becomes a rate-limiting factor. A simple, novel method has been developed to determine the thermal conductivity of polymer sheets by DSC. The slope (or intercept) of the melting peak of a metal placed on top of the polymer specimen and the slope (or intercept) of its melting peak in a bare pan are determined. Theory of heat flow in DSC has been developed so that the thermal resistance and time constants for thermal flux in the polymer specimens can be determined from these data. The method holds promise of becoming a simple, quick, and nondestructive method for thermal conductivity measurements of thermaliy insulative sheet materials.

Data from these new techniques are being applied to modeling the kinetics of photopolymerization reactions in close conjunction with work on the investigation of thermoset resin polymerization kinetics by infrared spectroscopy and rheological property measurements, which is also being undertaken in the Polymer Science and Standards Division. 
The most promising areas of growth in polymer markets are in construction, transportation, and electrical systems where long-term durability is essential to competitive performance. To compete successfully in these markets, our country needs scientific research on the mechanisms by which polymeric materials are degraded because of environmental and other imposed stresses. In fact, a recent National Research Council report states, "Mechanistic data on degradation are essential (1) in identifying possible stabilization processes to slow degradation, and (2) in developing shortterm methodologies for predicting service life."

Research this year on degradation mechanisms and rates includes investigations of the hydrolysis of polyester polyurethanes, the degradation of polyurethane tubings in media simulating body fluids, and the long-term stability of polyester-based recording media. In addition, we have carried out a collaborative program with the Building Materials Division to investigate the kinetics and mechanism of the photodegradation of PMMA, a polymer widely used for solar cover plates.

Protective additives are needed for fabrication and long-term use of most polymers. In use, these additives inevitably diffuse through the polymer to some extent and may migrate out of the polymer entirely. The polymer is then left with poorer properties or made more vulnerable to degradation and failure. This is a major failure mode of polymers, and lifetime prediction methods must explicitly include the rates of additive diffusion along with the rates of the inhibited and uninhibited degradation reactions. The number of commercially usable polymer-additive combinations is too large to be considered efficiently on a case-by-case basis. The emphasis in this task is, therefore, on the development of general principles or models of diffusion that are applicable to the widest range of generically important systems. In addition, knowledge of the general principles which control diffusion through solid polymers is important to the design and evaluation of improved additive systems. Work this year on the migration of additives includes measurements of diffusion rates by solvent extraction and by inverse chromatography and investigations of slow diffusion in solid polymers by fluorimetry.

Prediction of the Long Term Stability of Polyester-Based Recording Media

D. W. Brown, R. E. Lowry, and L. E. Smith

The stability of poly(ethylene terephthalate) is being studied for the National Archives in order to predict its long term behavior as the base for film and magnetic tape. The project is supposed to last for four to five years. A report has been written that contains results of the second year's work. Film base, photographic film, and electrographic film are being aged at several temperatures and relative humidities, RH. Acid contents, mechanical properties, and molecular weight have been measured at intervals. The rate of increase in acid content, $k$, provides a useful measure of the degradation rate. Data for films aged between 115 and $55^{\circ} \mathrm{C}$ at 100 percent RH obey the equation Ln $k=39.3-(14000 / T)$, where $k$ is in percent/day and $T$ is the absolute temperature. The water content is included in $k$, which is approximately proportional to RH. Lifetimes of the films appear to be equal to $69.3 / \mathrm{K}$ days. Extrapolation of 25 and $20^{\circ} \mathrm{C}$ gives 400 and 900 years for the lifetime at 100 percent RH. A more sensitive analytical method than now used will be required to make useful measurements of acid content at $35^{\circ} \mathrm{C}$, even after samples have been aged for five years. Two films have always failed before the others. The short-lived films might well be stored with others, since failure of the former would warn of the approaching failure of the latter.

Magnetic tapes are also being aged, primarily to study the binder that holds the magnetic oxide to the substrate. Binder on five brands of tape that were aged at $60{ }^{\circ} \mathrm{C}$ become cross-linked at RH of zero and 11 percent, changed little at 30 percent RH, but degraded at 100 percent RH. The binder on tapes aged at $85^{\circ} \mathrm{C}$ and 100 percent RH first degraded severely and then appeared to cross-link slightly. Badly degraded binder was not gummy but was easily detached from the substrate. Model binder aged at $85^{\circ} \mathrm{C}$ and 100 percent RH eventually became brittle.

\section{Hydrolytic Degradation of Polyester Polyurethanes}

D. W. Brown, R. E. Lowry, and L. E. Smith

A manuscript entitled "Equilibrium Acid Concentrations in Hydrolyzed Polyesters and Polyester Polyurethanes" was prepared and submitted to the Journal of Applied Polymer Science. Conclusions are the same as those in last year's report: namely, that equilibration does not set a practical 
limit to the hydrolytic degradation of thermoplastic polyurethane elastomers and that chemical rate constants calculated in the conventional manner appear to increase with the relative humidity.

Attempts were made to develop a general mechanism to fit data from the hydrolytic degradation of polyester diols in the presence of different concentrations of carbodiimide, an hydrolysis stabilizer. These. were not very successful, despite allowing for possible hydrolysis of the carbodiimide, hydrolysis of the acyl urea produced in the reaction of acid with carbodiimide, and uncatalyzed and acid-catalyzed ester hydrolysis. More data will be obtained with polyester polyurethanes before making additional attempts to modify the mechanism.

\section{Studies With Polyether Polyurethane Tubing}

\section{W. Brown, R. E. Lowry, and L. E. Smith}

Polyether polyurethane tubing is used for electrical insulation on pacemaker leads implanted in blood vessels. Failure of this tubing has been reported in the literature but the causes and the exact nature of the failure are in dispute. The Bureau of Medical Devices is sponsoring an in vitro aging study of the tubing to help guide their investigation of the extent and causes of any problem with the tubing.

Samples of tubing have macroscopic and microscopic irregularities that are indicative of orientation gradients. It is not known if these can lead to failure. There were too many irregularities to avoid incorporating some in any piece more than a few centimeters long, so samples were prepared without trying to select regions with and without the irregularities. Some samples were stretched on glass or Teflon rods in order to accelerate the aging. Extension ratios in the circumferential and axial directions were of the order of four and two, respectively. Other samples were bent into smail radius bends to. induce stresses. Eight different media were used, including air, water, Ringer's solution, bovine serum, and several mixtures containing lipids, bile salts, and hydrogen peroxide. The degradation was followed by measuring the molecular weight by gel permeation chromatography. The active media were water, Ringer's solution, and hydrogen peroxide. Only the hydrogen peroxide solutions affected unstretched samples. Comparison of results from samples on glass and Tefion rods indicated that the glass was chemically active, since degradation on the Teflon was only one third as great.

These experiments are being repeated using fewer media and a greater variety of stretching conditions. Only Teflon rods are being used to stretch the samples.

Solar Type Photolytic and Thermal Degradation of Plates of Poly(Methyl Methacrylate)

\section{B. Dickens}

A joint program between the Polymer Science and Standards Division of the Center for Materials Science and the Building Materials Division of the Center for Building Technology has provided a chemical mechanism for the destructive. effect of sunlight on a transparent glazing material. This information explains the deleterious effects of oxygen, unpolymerized raw material and high temperature, and is an important step in the achievement of estimates of lifetimes in service.

The mechanical properties of polymers are dependent on the lengths of the chain-like polymer molecules which must be above a certain value to confer useful properties. As damage processes reduce the average molecular weight of the polymer molecules, the polymeric material approaches and eventually reaches a condition in which it fails to maintain its function. Thus the effect of damage processes can be followed via measurements of molecular weight.

Plates of poly(methyl methacrylate), PMMA, were exposed at various temperatures and over a series of times to radiation with a solar-type spectrum. The specimens were sectioned parallel to the plate surface and analyzed by gel permeation chromatography to give precise estimates of the changes in molecular weight of the material. Changes were also followed using Fourier transform infrared spectroscopy, ultraviolet spectroscopy, and fluorescence spectroscopy and fluorescence lifetime measurements.

The results show the importance of the role of oxygen in reducing molecular weight, and of unpolymerized raw material in essentially converting the initial products of degradation into a form 
which is more sensitive to oxygen. Calculations based on the diffusion and consumption of oxygen during irradiation have lead to the discovery of a decrease in the protective nature of the so-called cage effect of the polymers with temperature. This discovery has important ramifications in that the protective nature of the cage effect can also be changed by plasticizing agents such as water, which is taken up readily in small amounts by PMMA. Further work on the effect of water and temperature cycling on the damage rates will begin soon. The long term aim of the program is to correlate molecular weight changes with the significant factors in the service environment so that changes in molecular weight in service polymers can be predicted. In this way, changes in the mechanical properties can be predicted and estimates of service life given.

\section{Evaluation of IC-Encapsulants}

\section{S. S. Chang and J. R. Maurey}

Various chemical, electrical, mechanical, and thermal properties of thermosetting polymeric encapsulation systems for microelectronic circuits were evaluated. These properties were evaluated along with the characteristics of UV erasable programmable read-only memories and electrically erasable programmable read-only memories.

\section{Variable Heating Rate Thermal Analysis}

\section{J. H. Flynn}

The isoconversional method for determining energies of activation is used extensively for kinetic analysis of thermoanalytical experiments at a constant heating rate. Its popularity is due mainly to its capability of yielding activation energies without the necessity of one positing what often turns out to be incorrect models for the reaction mechanism. The use of Doyle's linear approximation of the temperature integral in this method introduces sizable errors in the calculation of the activation energy under certain conditions. The size and nature of these errors have been reviewed and a procedure has been developed for correcting them. These corrections have been found to be significant for cases where the activation energy is less than $100 \mathrm{~kJ} / \mathrm{mol}$. Examples of such cases include heterogeneous catalysis, biological reactions, and reactions where the rate limiting step is the diffusion of small molecules through their evaporation from a condensed phase.

\section{Measurement of Additive Diffusion by Fluorimetry}

F. W. Wang, B. F. Howe 11, and F. L. McCrackin

Antioxidants and other additives are added to polyolefins and other plastics to improve the useful properties and to extend the service life of plastics by preventing or retarding degradation. The effectiveness of these additives depends not only on their chemical properties but also on their ability to remain in the plastics throughout the intended lifetime under various end-use conditions. The basic physical quantities which control the retention of additives in plastics are their diffusion coefficients and solubilities. Thus, measurements of diffusion coefficients and solubilities are important for effective design of additive-polymer systems.

We have carried out, by fluorimetry, both extraction experiments and concentration-profile experiments to determine the diffusion coefficients of additives in low-density polyethylene.

In an extraction experiment, a plane sheet of polymer with uniform concentration of a fluorescent additive is immersed in a limited amount of well-stirred solvent. Then, the amount of the additive extracted by the solvent is determined at various times by fluorimetry. 'The diffusion coefficients of an amine antioxidant, $N, N^{\prime}$-diphenyl-p-phenylenediamine (DPPD), and a substituted thiophene UV screener, 2,5-di(5-tert-butyl-benzoxazoly1)thiophene (Uvitex $O B$ ) in low-density polyethylene have been determined from extraction experiments.

At the beginning of a concentration profile experiment, twenty polyethylene films without additive, 4 mils thick and 1.5 inches in diameter, were stacked in the center of a diffusion device. A porous teflon disc which was 1.5 inches in diameter and filled with a fluorescent additive was then placed above and below the stack and served as a reservoir of additive. The assembly was clamped between two heavy aluminum plates and the whole assembly was then placed in a nitrogen filled chamber at $30^{\circ} \mathrm{C}$. As time passed, the additive diffused from the teflon discs into the 
polyethylene films. At the end of an experiment, the polyethylene films were separated, and the concentration of the additive in each film was determined by fluorimetry. The raw data thus obtained were the values of concentration as a function of position after a fixed length of time. By comparing the concentration profile with master curves obtained by solving the diffusion equation with boundary conditions, we can determine the solubility and the diffusion coefficient of the additive in polyethylene. In this way, we have obtained a preliminary value for the diffusion coefficient of 3-tert-butyl-4-hydroxyanisole(BHA) in polyethylene at $30^{\circ} \mathrm{C}$.

\section{Migration of Low Molecular Weight Materials in Polymers Under Simultaneous Swelling Conditions}

\section{M. Guttman and S. S. Chang}

Additives are placed in polymer films to enhance their properties, improving the polymer's chemical stability as well as the polymer's mechanical properties. The loss of the additives from the polymer films into liquids in contact with the polymers not only causes degradation of the films properties but also often contaminates the liquids causing uncertain health hazards. Thus the loss of additives out of polymer films is an important area of study.

In earlier work in this laboratory Chang and coworkers have shown that the mass loss of an additive from a semicrystalline polymer into a solvent that swells the polymer does not obey Fick's law. They found that at early times of the extraction experiment, the amount of the additive extracted into the solution at time $t, M(t)$, increases as the square root in time. At intermediate times, $M(t)$ increases more rapidly (almost linearly) with time. Finally, at later times, $M(t)$ again increases like the square root of time before the final approach to equilibrium. The diffusion coefficient at long times is found to be much larger than that observed at earlier times. In contrast, Chang found that mass loss of additives from polymers into solvents which do not swell the polymer followed normal Fickian behavior.

Current work is directed at the development of a theoretical model to explain the mass loss into solvents which swell the polymer. The model being studied relates the non-Fickian migrant loss behavior to the non-Fickian sorption behavicr. In particular, we assume that the migrant diffuses with diffusion constant $D_{1}$ in the swollen region and with diffusion constant $D_{2}$ in the unswollen region. $D_{1}$ is greater than $D_{2}$. We assume that the swelling front is sharp and moves proportional to time (type two diffusion). Thus it is necessary to solve the one dimensional diffusion equation in which there are two regions each having a different diffusion constant and in which the width of each of the regions changes as a function of time.

Exact analytical solutions for such a problem have so far not been possible. However, asymptotic solutions for very short time and for very long times show the limiting behavior seen in the experiments. Furthermore, an approximate analytical solution has been produced which show most of the general features of the experimental data of Chang. In these studies the velocity of swelling was estimated from swelling data on polyethylene obtained by Rodgers et al. and $D_{1}$ and $D_{2}$ obtained from the data of Chang. Currently a numerical solution to the problem is being undertaken.

\section{Migration of Styrene Monomer From Polystyrene}

\section{S. S. Chang and J. R. Maurey}

The migration of styrene monomer from polystyrene into various solvents has been observed by radioactive tracer techniques. The content of styrene in the polymer is nominally 0.4 percent.

n-Heptane distorts the sample quickly and therefore should not be considered as a food-oil simulating solvent, although it may be used effectively to characterize the styrene content of the sample.

Most other experiments involving corn oil and ethanolic solvents took more than one month before reaching equilibrium extraction. For corn $0 i 1$ at $30^{\circ} \mathrm{C}$, less than 10 percent of styrene was extracted even after one year. In these experiments, the migration behavior may be described by two Fickian regions, with the longer-time diffusion coefficient greater than the initial diffusion coefficient. 
The behavior of migration under the influence of solvent swelling for both glassy and semicrystalline polymers is being modeled in collaboration with C. M. Guttman.

\section{Chromatographic Studies of Migration From Polymers}

\section{G. A. Senich}

The method of polymer analysis termed inverse phase gas chromatography (IGC) differs from conventional gas chromatography in that a polymer of interest is employed as a stationary phase which sorbs various volatile organic species, often referred to as probes. The experiment involves injecting the probe and a gaseous marker into a flowing carrier gas stream which propels the substances through a column containing the polymer, coated on an inert support packed within the column. The time lag between injection and elution of the probe vapor peak and its standard deviation characterize the polymer-probe interactions, which are strongly influenced by the carrier gas flow velocity. The steady-state probe distribution reached between vapor and polymer phases at low flow rates allows polymer-probe thermodynamics to be studied. A steady-state distribution is not reached at high flow rates where the kinetics of polymer-probe interactions are accessible and the probe diffusivity in the polymer can be obtained. The probe concentration in the polymer is normally very low and the technique lends itself to determinations of the degree of migration of trace additives, oligomers, and polymerization residues from polymers.

The extent of migration of a sufficiently volatile material from a polymer into a contacting substance (typically a solvent) can be estimated from data readily obtained by IGC studies using the migrant or additive as the probe. The specific retention volume is obtained experimentally from the time lag at slow carrier gas flow rates and is used to determine the polymer-migrant interaction parameter. When combined with a measured or literature value of the solubility of the migrant in the solvent, a calculation of the equilibrium partition coefficient is possible. The latter is a direct measure of the fraction of the additive which can be expected to migrate from the polymer to the contacting medium. If the time over which migration can occur is short, an equilibrium prediction may greatly exaggerate the degree of migration expected. A value for the diffusion coefficient of the migrant in the polymer may then be obtained from the peak standard deviation of IGC experiments and employed to estimate the degree of migration which will occur over the shorter time period. Experimental determinations by IGC of the extent of migration of protective additives from polymers are continuing. Both thermodynamic interactions and diffusion behavior are of interest.

\section{Solubilities and Partition Coefficients of BHT in Polyolefins and} Various Solvents

\section{S. S. Chang and J. R. Maurey}

BHT(3,5-di-t-Butyl-4-hydroxytoluene) is one of the most widely used antioxidants for polyolefins and many other polymers. The solubility of BHT in aqueous media is generally in the ppm level and therefore requires either sensitive chromatographic techniques or radiotracer techniques. However, as BHT degrades readily in the presence of water, the true value of solubility in aqueous media can only be estimated by extrapolating the observation to time zero. BHT is readily soluble in many organic solvents, and is relatively stable in organic solution.

The solubility of BHT in polyolefin is a more controversial subject. The apparent solubility of BHT in polyolefin is relatively small. However, there exist literature values indicating moderate solubility from vapor absorption studies and very high solubility from diffusion studies. We are attempting to solve this controversy through partition coefficient studies and direct observation of the solubility in condensed phases.

\section{Determination of Segmental Mobility by Picosecond Fluorescence Spectroscopy}

F. W. Wang, R. R. Cavanagh ${ }^{1}$, and W. H. Grant

${ }^{1}$ Molecular Spectroscopy Division

The study of polymer-segment mobility is of practical as well as scientific interest. Such a study will on the one hand contribute to the design of polymeric reagents and on the other hand, 
shed some light on "internal viscosity," an idea introduced by Kuhn some 35 years ago and yet still argued about $[1,2]$. Therefore, we have started a project to measure polymer-segment mobility by picosecond fluorescence spectroscopy.

An excimer is formed by the association of an excited molecule with another molecule in its ground state. Such an excimer gives off a broad structureless fluorescence which has longer wavelengths than the fluorescence of the isolated excited molecule. Excimers may also be formed intramolecularly from molecules carrying excimer-forming groups. For example, excimer fluorescence has been observed in dilute solutions of pyrene-labeled alkanes such as 1,3-bis-(1-pyrene)propane and 1,10-bis-(1-pyrene)decane [3].

In a novel experimental technique for studying segmental mobility, the solution of an excimerforming oligomer or polymer is irradiated with pulses of light at $355 \mathrm{~nm}$ with pulse width of $27 \mathrm{ps}$. Since a pyrene excimer, for example, is formed by the diffusion-controlled reaction of an excited pyrene group and a pyrene group in the ground state, the rise of pyrene-excimer fiuorescence intensity will be faster if the pyrene groups are attached to more mobile segments. Similarly, the more mobile the segments, the faster the decay of pyrene-monomer fluorescence intensity.

We have applied the picosecond fluorescence technique to measure the segmental mobility of 1,3-bis-(1-pyrene)propane, 1,10-bis-(1-pyrene)decane, copolymers of 1-pyrenylmethyl methacrylate and methyl methacrylate, and poly(1-pyrenylmethyl methacrylate). We have found that the pyrene groups of the pyrene-labeled homopolymer and copolymers are more mobile than those of the pyrene-labeled al kanes.

[1] Ott, H.; Cerf, R.; Michels, B.; Lemarechal, P. Chemical Physics Letters, 24, 323 (1974).

[2] Stockmayer, W. H. Pure Appl. Chem. Supplement, 8 , 379 (1973).

[3] Zachariasse, K. A.; Kuhnle, W. Z. Physik. Chem. NF, 101, 267 (1976). 
The goal of the task on electrical properties is to carry out long range research to provide the basis for new or improved measurements and provide concepts and data which will lead to higher performance in the electrical applications of polymers. The research encompasses a broad range of generic applications from insulating materials with low loss to materials that conduct either electrons or ions. An important part of the program includes research on polymers that can be made to exhibit piezoelectric and pyroelectric properties which has led to new types of transducers and new measurement capabilities. Results of the research in the form of data, measurement techniques, and scientific concepts go directly to funding organizations through written reports or in some cases by the delivery of a measurement system. The information is made available to interested parties in the general public by publication in archival journals, by presentation at the meetings of technical societies, through research associates who come to work with us, and by visits to our laboratories.

Polymers have long been used as electrical insulators where they have performed well. However, higher demands are being made on their electric breakdown strength as the electric power companies increase the voltage of power transmission, and make requirements for longer lifetime in more hostile environments as the trend to underground cables continues. A measurement technique developed to probe the distribution of polarization in polymer films offers an opportunity to measure the distribution of charge in insulation film and thus provide information on the role of non-uniform electric fields in determining the breakdown strength of polymers. The time-domain dielectric spectrometer which was recently completed can be used to readily follow any changes in dielectric loss as a polymer is exposed to use conditions.

Electric conduction in organic polymers is an exciting new area of solid state physics and chemistry. It is generally agreed that a conjugated double bond structure along the polymer chain is a necessary condition for conducting properties but the role of the "dopants" which must be added to induce conductivity is still being investigated. The porous nature of polyacetylene, its ability to be oxidized and/or reduced by "dopants" such as metals, and its resultant conducting properties offer great opportunities for battery electrodes and electrochemical sensors. This field of investigation requires data on molecular packing, dopant location, chemical changes with doping, and charge carrier measurements to form a basis for modeling and predicting electrical properties. Fast ion transport in polymers provides a basis for a large and growing class of important applications including the production of chlorine and sodium hydroxide from the electrolysis of brine, hydrogen production from low-head hydroelectric sources, solid electrolytes for fuel cells and batteries, materials separation membranes and electrochemical sensors. These polymers differ from conducting polymers referred to above because the carriers are ions, not electrons. In most applications it is required that the current be carried entirely by ions participating in an electrochemical reaction rather than electrons which essentially short circuit the cell. NBS work involves the preparation of ion-conducting specimens, measurement of electrical properties, characterization of structure, and relating the structure and properties to provide a basis for transport mechanisms at the molecular level. These concepts will then guide the preparation of additional ion-transporting films.

Research at NBS has made a significant contribution to the understanding of the mechanism responsible for the piezoelectric and pyroelectric properties of some polymers. Applications for these new transducers are continuing to grow and there is a need for predicting their long term performance through an understanding of the mechanism whereby polarization is lost at elevated temperatures. Factors influencing the stability such as poling conditions, crystal structure, chemical structure, and molecular motions are being investigated. In addition to inproving our understanding of these polymers, the polymeric transducers are being fabricated and employed in instrumentation to solve measurement problems posed by other government agencies.

\section{Automated Dielectric Measurements}

\section{F. I. Mopsik}

This activity was undertaken to provide a more efficient, rapid, and at the same time highly accurate and sensitive measuring system for measuring the dielectric constant and loss of dielectric material at audio and subaudio frequencies. 
The instrument, a dielectric spectrometer, records the transient electric charge response to a step change in applied voltage as a function of time from 5 ss to up to $3000 \mathrm{~s}$. The charge response is then numerically transformed into the dielectric constant and loss for an equivalent frequency range from $10^{-4}$ to $10^{4} \mathrm{~Hz}$. The basic accuracy of the instrument is 0.1 percent in tan $\delta$ with resolution in tan $\delta$ to as $10 w$ as $10 \mathrm{~s}$.

In FY83 the instrument was completed and operated successfully. Real time graphics have been introduced so that the experimenter can monitor the course of the measurement while it is in progress. This last feature was found to be necessary when longer measurement times are used. I $t$ also allows the rapid, graphical presentation of data for analysis and experimental guidance. Experience in FY83 has shown the instrument to be reliable, easy to use, and a great improvement over older methods.

A paper on the instrument has been completed and submitted to Review of Scientific Instruments for publication. Research associates from General Electric Company have started a transfer of the technology to their laboratory for use in research. Another research associate program with IMASS, Inc. has been started to produce a commercial version of the instrument.

\section{Dielectric Properties of Polyvinylidene Fluoride}

\section{A. J. Bur and F. I. Mopsik}

This subtask directly supports the optimization of piezoelectric polymers for applications in industry and Government. It concerns the definition of PVDF properties prior to poling which, after poling, wil! yield highly active transducers with optimum ageing characteristics.

Using the dielectric spectrometer referred to above, the dielectric properties of PVDF were measured over a frequency range of $10^{4} \mathrm{~Hz}$ from room temperature to $90{ }^{\circ} \mathrm{C}$. The data at room temperature were supplemented by measurements on a high frequency bridge to extend the range to $10^{8} \mathrm{~Hz}$. At room temperature, four dielectric loss regions were observed: a high frequency loss at $10^{6} \mathrm{~Hz}$; a loss at $1 \mathrm{~Hz}$; an intermediate loss region at $10-2-10^{-3} \mathrm{~Hz}$; and a low frequency loss region at frequencies less than $104 \mathrm{~Hz}$. With the exception of the $1 \mathrm{~Hz} \mathrm{loss,} \mathrm{which} \mathrm{occurs} \mathrm{only} \mathrm{in}$ samples containing an unoriented $\alpha$ phase, the observed dielectric dispersions were present in all samples regardless of their preparation history. The $10^{6} \mathrm{~Hz}$ dispersion is known to be associated with the rubber-to-glass transition $\left(\mathrm{Tg}=-50{ }^{\circ} \mathrm{C}\right)$ and therefore is attributed to micro-Brownian motion of molecules in the amorphous phase. Usually, in semicrystalline polymers dispersions which occur at frequencies lower than (or temperatures higher than) the rubber-to-glass transition are attributed to the crystalline phase. The situation with PVDF appears to be an exception to the rule. For example, the $1 \mathrm{~Hz}$ dispersion and the $10^{6} \mathrm{~Hz}$ loss peaks are complementary, i.e., a decrease in one results in an increase in the other. For an $\alpha$ phase sample, the $1 \mathrm{~Hz}$ peak decreases in intensity upon orientation but its amplitude is absorbed into the dispersion at $10^{6} \mathrm{~Hz}$. This is observed for both $\varepsilon^{\prime}$ and $\varepsilon^{\prime \prime}$. Thus, the $1 \mathrm{~Hz}$ dispersion appears to be associated with the amorphous phase. Its molecular mechanism has not been determined but we suggest that it is associated with the interface between amorphous and crystalline phases. Previous assignment of this peak to a relaxation of the component of the dipole moment parallel to the main chain ( $\mu_{11}$ ) in the $\alpha$ phase crystal appears to be incorrect. The intermediate frequency loss region $\left(10^{-2}-10^{-3} \mathrm{~Hz}\right) \mathrm{differs}$ in magnitude for $\alpha$ and $\beta$ phase samples and is attributed to the crystalline phase. The very low frequency loss (less than $10^{-4} \mathrm{~Hz}$ ) contains a broad distribution of relaxation times and is not entirely accounted for by DC conduction. At elevated temperatures conductivity dominates the dispersion data in this frequency range but at room temperature a long-time relaxation is clearly seen.

The dielectric samples have been characterized by $x$-ray and density measurements. From these data, we are able to calculate the fractional component of the $\alpha$ phase, $\beta$ phase and amorphous phase in each sample. The data show that the amorphous fraction remained constant for all samples at 52 percent. Using $x$-ray orientation data for oriented $\alpha$ phase samples, the component of $\mu_{31}$ which is perpendicular to the axis of sample orientation was calculated. (This is the component which would contribute to the dielectric dispersion). It was found to be too small to account for the $1 \mathrm{~Hz}$ loss. 


\section{A. J. Bur}

This work was undertaken as a result of a direct request from the $U$. S. Army Civil Engineering Laboratory. It consists of a review of dielectric loss data for polymers at microwave frequencies and an elucidation of the molecular mechanisms which are responsible for microwave absorption.

Archival journals and laboratory reports were searched in order to obtain the available microwave dielectric data. While the main focus of attention was the frequency range from $100 \mathrm{MHz}$ to $100 \mathrm{GHz}$, loss spectra outside this region were also reviewed because variations in temperature can cause a shift of dielectric loss into or out of the microwave range. A large volume of data for low loss polymers (polyethylene, polypropylene, and polytetrafluoroethylene), which are used in the communications industry, was available for review. Other polymers, for which data were available and which have significant loss at microwave frequencies, are: polystyrene, polyvinyl chloride, polyvinylidene fluoride, polymethyl methacrylate, polymethyl acrylate, polyoxymethylene, polyethylene oxide, polypropylene oxide, polyacetylene, and polysulfur nitride. The origins of microwave dielectric loss in polymers are categorized as: (a) dipolar absorption dispersions in both crystalline and amorphous polymers; (b) dipolar losses due to impurities, additives or fillers in a polymer material; (c) microwave absorption in conducting polymers (polyacetylene and polysulfur nitride) for which the current carriers are electrons; and (d) photon-phonon absorption spectra corresponding to the density of states in amorphous regions of a polymer material. Non-polar polymers, such as polyethylene, have low dielectric loss at microwave frequencies. Amorphous or semicrystalline polar polymers, which have a glass transition temperature less than $-50^{\circ} \mathrm{C}$, can display large microwave loss at room temperature.

This review has been completed and a final report has been delivered to the Army.

\section{Conducting Polymers}

C. K. Chiang

The physical properties of polyacetylene, $(\mathrm{CH})_{x}$ when doped closely resemble those of conventional semiconductors. The chemical behavior of doped $(\mathrm{CH})_{x}$ is in many aspects analogous to that of insertion compounds. The highly conducting $(\mathrm{CH})_{x}$ polymer can incorporate as much as 10 to $20 \mathrm{~mol} \%$ of the dopants into its structure. The high conductivity and the ability to react with large amounts of dopants make the $(\mathrm{CH})_{x}$ a good candidate for electrode material. Studies on the $(\mathrm{CH})_{x}$ electrodes in liquid cells have been reported. In the past year we have studied $(\mathrm{CH})_{x}$ using solid containing dopants, and on the use of the $(\mathrm{CH})_{x}$ electrode in a solid state cell.

When polyacetylene acts as an electrode in a solid state cell, the electrode process is the doping and undoping of the $(\mathrm{CH})_{X}$. The solid state doping of $(\mathrm{CH})_{X}$ is studied using a three-

electrode cell with two $(\mathrm{CH})_{x}$ electrodes and a sodium electrode in the polyethylene oxide (PEO) complex conductor arranged as follows:

$$
\text { (CH) } x\left|\frac{\mathrm{Na}}{\mathrm{Na} / \mathrm{PEO} \text { Complex }}\right|(\mathrm{CH})_{x}
$$

where two polyacetylene electrodes are suitably arranged so that the ac impedance of $(\mathrm{CH})_{x}$ can be measured in situ without removal from the cell. The doping process is started by connecting a negative voltage to both $(\mathrm{CH})_{x}$ film electrodes and a positive voltage to the sodium electrode. The current carried by sodium ions flows from the sodium electrode to both $(\mathrm{CH})_{x}$ film electrodes. The sodium ions arrive at the $(\mathrm{CH})_{x}$ film and react with it electrochemically to form $\left(\mathrm{CH}-\mathrm{Na}_{y}\right)_{x}$, where $y$ is the concentration of sodium ions in doped $(\mathrm{CH})_{x}$. The total charge transferred during the process is proportional to $y$. The effect of doping can be measured by the change of resistivity of the doped $(\mathrm{CH})_{x}$ film electrode, which is obtained from analyzing the ac impedance data. 
The resistivity of $(\mathrm{CH})_{x}$ in the electrode before doping is $1 \times 10^{6} \mathrm{ohm}-\mathrm{cm}$. On doping with sodium ions the resistivity increases initially. With further doping, the resistivity reaches a maximum and then decreases slowly. At the end of the doping experiment the resistivity of $(\mathrm{CH})_{x}$ decreases by over three orders of magnitude.

Our preliminary data indicate that the doping can proceed in a solid state cell. To examine the electrode behavior the emf of $\mathrm{Na} /(\mathrm{CH})_{x}$ cell versus sodium concentration is measured using the cel1

$$
\text { (CH) } x \text { PEO-NaI Complex } / \mathrm{Na}
$$

In this case, the cell voltage for pure $(\mathrm{CH})_{x}$ is 2.7 volts and decreases with increasing dopant concentration. The results resemble those reported for the highly successful insertion compound $\mathrm{Li}_{\mathrm{TSS}}$.

Fast Ion Transport in Solid Polymers

C. K. Chiang, G. T. Davis, C. A. Harding, and T. Takahashi ${ }^{1}$

${ }^{1}$ Guest worker from Ube Industries, Japan

Polyethyleneoxide (PEO) with added alkali metal salts has been shown to exhibit unusually large conductivities--especially at temperatures around $120^{\circ} \mathrm{C}$. A complex is formed between the polymer and the salt which increases the melting point from $60^{\circ} \mathrm{C}$ for PEO to the vicinity of $190{ }^{\circ} \mathrm{C}$; the exact temperature depends upon the particular salt. The formation of the high melting complex imparts structural rigidity to films of the material at temperatures above the melting point of pure PEO. There are also several reports in the literature that ascribe the mobility of the cation to a specific pathway of conduction along the interior of the $3 / 1$ helical chain conformation which the polymer assumes in the crystalline phase. The activation energy for conduction as obtained from Arrhenius plots changes markedly at a temperature which is close to the melting point of the uncomplexed polymer--becoming smaller at higher temperature. Understanding the mechanism of ionic transport in this polymer is essential in guiding efforts to select or synthesize other polymers which conduct in the solid state.

During the past year, we have started an experimental program in which we first duplicated the results of previous investigators on preparing PEO-salt complexes by evaporation of a common solvent but have also shown that crystals of PEO grown from dilute solution can be converted to a PEO-NaI complex by exposing the crystals to a solution of $\mathrm{NaI}$ in amylacetate. Evidence for the complex comes from DSC and x-ray data. The thermal behavior of PEO-NaI mixtures as revealed by differential scanning calorimetry (DSC) is dependent upon salt concentration and thermal nistory. Below 10 mole percent $\mathrm{NaI}$, only one endothermic peak appears at the normal melting point of PEO, i.e., $60^{\circ} \mathrm{C}$. For concentrations of 15 percent and greater, the first heating following evaporation of the solvent exhibits a rather irregular trace with a broad endotherm in the vicinity of $190{ }^{\circ} \mathrm{C}$. After having been melted and cooled, subsequent traces show two endotherms, one near $60^{\circ} \mathrm{C}$ and another near $190^{\circ} \mathrm{C}$ which are regular and reproducible upon subsequent heatings. It has also been determined that the NaI separates as a separate phase above the melting point of the complex.

DC conductivity is inferred from data on complex impedance measured over a wide range of frequency or from 4-terminal DC measurements. By studying a wide range of $\mathrm{NaI}$ concentrations in PEO rather than the "stoichiometric ratio" commonly employed, we have shown a maximum in conductivity at low salt concentration (ca. 2 mole percent) which is below the level required to see any evidence for the PEO-NaI complex. Therefore, the concept of a specific pathway for ionic conduction may not be necessary to explain the transport process in this polymer.

With the aid of a guest worker from Ube Industries in Japan, we have synthesized linear polyethyleneimine $\left(-\mathrm{CH}_{2} \mathrm{CH}_{2} \mathrm{NH}-\right)$ and have shown that it too can form a high melting complex with $\mathrm{NaI}$ but once melted, it does not reform upon cooling. Preliminary results on the conducting of the PEI-NaI preparation indicates that it is not as good a conductor as the PEO counterpart but the early results are very encouraging. We intend to convert this polymer to the quaternary ammonium analog to examine the role of the groups on the nitrogen in influencing ion transport. 


\section{G. T. Davis and M. G. Broadhurst}

The objectives of this task are to study the molecular motions, structural changes, and electrical properties associated with the process whereby polymers are made piezoelectric. Information gained from these studies will be used to optimize piezoelectric and pyroelectric response with respect to both initial activity and long-term stability.

While piezoelectric and pyroelectric activity from polymers is comparable to that from good ceramic materials, other properties of polymers often make them the preferred transducer material. Some of the polymer properties which make them so useful include toughness, flexibility, low density, broad band response, close acoustic impedance match to water, and ease of fabrication. Requests for information from other Government agencies and from industries have remained numerous. Interest in this area of research continues at a high level in the U. S. as well as Japan, England, and Europe. Many papers dealing with piezoelectric polymers were presented at the recent IEEE International Symposium on Applications of Ferroelectrics, a special conference on medical applications of piezoelectric polymers was held in Pisa, Italy and piezoelectric polymers was the subject of a U. S. --Japan cooperative science seminar held in July of 1983.

Collaborative work on copolymers of vinylidene fluoride and trifluoroethylene has continued with Bell Laboratories and the Institute of Physical and Chemical Research in Saitama, Japan. Compositions containing $52,65,73$, and 78 mole percent of vinylidene fluoride (VDF) have been studied. A ferroelectric to paraelectric transition involving a change in chain conformation within the crystal occurs in all of these compositions but the temperature of the transition increases with increasing VDF content. Hysteresis between electric displacement and electric field shows that these copolymers can be poled at much lower electric fields that the PVDF homopolymer and piezoelectric activity can be comparable even without orienting the polymer chains prior to poling. Phenomena related to ionic conduction and trapping of charge have been observed in these copolymers when subjected to poling fields at temperatures of $70^{\circ} \mathrm{C}$ or greater. A reduction in the magnitude of polarization reversal occurs as a result of these charges but hysteresis is restored if the copolymer is heated to the paraelectric phase and returned to room temperature. The influence of such charges and the internal fields they create on the thermal stability of polarization is now being investigated.

\section{Polarization and Space Charge Studies}

\section{A. S. DeReggi and F. I. Mopsik}

The thermal pulse technique has been developed in this laboratory for studying the distribution of permanent poiarization across the thickness of a dielectric film. This measurement method has provided key information in understanding the poling behavior of polymeric materials used as piezoand pyroelectric elements.

The method has been applied to the study of the poling of PVDF at room temperature. The application of forward and reverse poling fields in sequence showed that the regions of the film near the surfaces require higher poling fields than the bulk of the film to acnieve the same polarization. Typically the surfaces remain less poled relative to the center by approximately 15 percent at fields of $2 \mathrm{MV} / \mathrm{cm}$. When a film was first poled to $2 \mathrm{MV} / \mathrm{cm}$ and reverse poled to $-1 \mathrm{MV} / \mathrm{cm}$, the center was reverse poled but the surfaces still retained polarization in the original direction with nearly zero mean polarization for the entire sample. A paper on these results has been accepted for publication in Applied Physics Letters.

A study in collaboration with Professor Sidney Lang of Ben Gurion University has continued. PVDF was poled at low fields at room temperature with $A l$ and $A u$ electrodes to see if work function changes in the electrode materials would influence the poling behavior. Previous work indicated that this could be so. A detailed series of measurements showed that this was not the case but apparent changes were due to the presence of a small amount of permanent polarization in the original, nominally unpolarized film. The strength of this preexisting polarization varied randomly among samples. Also, the two surfaces of the film were found not to be equivalent. These results reinforce and amplify our previous results that the film is intrinsically nonuniform in its poling behavior. A paper on these results has been accepted for publication in the Journal of Applied Physics. 
Because scientists in other laboratories are working on alternative approaches to the measurement of polarization distributions, and have recently begun to publish data on these, there. is a need for comparison of the results of different methods. This need has led to a collaboration with Bell Telephone Laboratories. Polarization distribution measurements on the same samples arc underway using both the thermal pulse and the acoustical pulse methods.

\section{A Dynamic Polymer Pressure Transducer With Temperature Compensation}

\section{A. J. Bur and S. C. Roth}

At the request of the U. S. Air Force, a pressure transducer using polyvinylidene fluoride (PVDF) as the active element is being developed for the purpose of measuring dynamic pressures in various soil environments underground.

Since underground pressure waves will be accompanied by adiabatic thermal effects, a transducer with temperature compensation is being developed. The essential elements of this gage are: (a) the active PVDF transducers; (b) a thermocouple having a short rise time; and (c) amplifiers which add the transducer and thermocouple in the appropriate proportion in order to achieve compensation for the temperature effects. Its construction involves laminating four sheets of $12 \mu \mathrm{m}$ PVDF together. The inner two sheets contain active areas $1 \mathrm{~cm}$ in diameter which are placed face-to-face with each other and on which are deposited aluminum electrodes. The outer two layers serve as protection for the inner two. A copper-constantan thermocouple junction, made with $75 \mu \mathrm{m}$ ( 3 mil) wire, is placed between the inner two sheets and within $2 \mathrm{~mm}$ of the active transducer area. For a pressure wave which is also accompanied by a temperature pulse, the active PVDF will respond to both pressure and temperature.

The thermocouple will respond to temperature only. By taking the output of the thermocouple and amplifying it in proportion to the pyroelectric coefficient of the PVDF, the thermocouple voltage can be used to correct the pressure wave signal from the transducer.

An amplifier has been constructed which can be used to achieve temperature compensation over the frequency range from $1 \mathrm{~Hz}$ to $10^{4} \mathrm{~Hz}$. Sensitivity to temperature changes of $0.2{ }^{\circ} \mathrm{C}$ and pressure changes of 1 psi $\left(7 \times 10.3 \mathrm{~N} / \mathrm{m}^{2}\right)$ have been obtained.

After testing the transducer in different soil environments, we will deliver six transducers to the Air Force.

\section{Acoustic Sensors}

\section{A. S. DeReggi}

The outstanding advantages of ferroelectric polymer transducers when used in hydrophones or in biomedical sensors of various types include negligible perturbation of the acoustic field measured, broad operating bandwidth, and mechanical flexibility. In addition, when polymers are used to make monolithic arrays, their mechanical properties are favorable for achieving low crosstalk between individual elements of the arrays.

To understand the general features of the performance characteristics which are peculiar to poiymeric transducers in terms of their material properties, we have considered the mixing of piezo and pyroelectric effects in pressure measurements. This mixing arises from the large adiabatic compressional heating coefficient which is typical of polymers. A paper on this topic has been presented at the 1983 International Symposium on Applications of Ferroelectrics.

A collaboration with the Brown University Foundation and the University of Pisa which started with a research associateship has had, as an outgrowth, the organization of the International Symposium on Piezoelectricity in Biomaterials and Biomedical Devices. A paper on the biomedical applications of piezoelectric polymers was presented at the meeting which took place June 20-23, 1983 in Pisa. It is anticipated that this first meeting will be followed by other meetings initially at two-year intervals.

During this fiscal year, we have initiated a collaboration through a research associate agreement with Alta Technology of Stamford, Connecticut. We have begun to study the factors affecting crosstalk in polymeric transducer arrays, and to explore ways to minimize this coupling in 
the design of high fidelity ultrasonic imaging arrays. One aspect of this work is aimed at investigating the applicability of trapped energy mode concepts in monolithic transducer arrays made with polymeric active material. Previous work elsewhere has shown that useful levels of isolation can be attained in monolithic transducer arrays made with ceramics if these arrays are operated in the frequency range where trapped energy modes prevail.

\section{Spontaneous Polarization in Ferroelectric Polymers}

\section{A. S. DeReggi, S. C. Roth, G. T. Davis, and M. G. Broadhurst}

A method for measuring the pyroelectric response of polymers as a function of temperature while the temperature is increased linearly with time has been developed at NBS. The technique provides a rapid means of determining the temperature at which polarization begins to decay and is being used to establish the effects of poling variables and polymer morphology on the relative stability of piezoelectric and pyroelectric properties.

Measurements of the sample polarization temperature dependence have been performed on PVDF and on its copolymer with tetrafluoroethylene (TFE). In both cases the polarization has been found to go to zero when melting of the sample is complete, rather than at some transition temperature distinct from the melting temperature. Measurements such as those described here must be done with well characterized samples since the ferroelectric polymers, like other semicrystalline polymers, may exhibit relaxation or melting behavior which depend on the prior mechanical and thermal history. The extended range of temperature over which melting may occur, and the multiphase nature of pVDF samples also must be considered in making the connection between the sample polarization and the spontaneous polarization.

The measurements on biaxially oriented PVDF poled at $100{ }^{\circ} \mathrm{C}$ show a gradual loss of sample polarization starting at $70^{\circ} \mathrm{C}$ and extending throughout the remainder of the semicrystalline range which finally melts at approximately $170^{\circ} \mathrm{C}$. There is no evidence of ferroelectric to nonferroelectric transition as reported by others, and no sign of impending precipitous polarization decrease of the kind shown by analogous spontaneous magnetization of a ferromagnet near a Curie point. Experiments with samples differing only in their poling treatment show generally similar polarization versus temperature behaviors. These results suggest that the polarization loss mechanism is not strongly influenced by the method of poling. Companion measurements of the angular dependence of the $x$-ray scattering intensity have been performed in reflection as a function of temperature from ambient temperature to the melting point. The results do not support an explanation for the loss of polarization in terms of a gradual phase transformation from the polar to antipolar phase $(\beta \rightarrow \alpha)$. Such a transformation was an admissible possibility since an $\alpha \rightarrow \beta$ transformation occurs upon mechanically orienting PVF 2 , and since a relaxation of mechanical orientation could be expected on heating.

Future measurements are planned on the VDF-trifluoroethylene copolymer at high VDF content. A structural-ferroelectric transition distinct from melting is observed im this system at a temperature which depends on composition and possibly on history. Extrapolation of the transition temperature vs composition data to 100 percent VDF would place the transition in PVDF in the area of its melting point. Future measurements also are planned for poly(vinyl fluoride) which is a semicrystalline system with a single crystaliine phase, in which transformation to alternate phases should not exist. 


\section{Invited Talks}

The Role of Interlaboratory Test Programs

in Quality Assurance ASTM Symp. on Quality

Assurance in Plastics,

Nashville, TN

J. D. Barnes

March 1983

Computerized Data Acquisition and

Control for Gas Transmission

Measurements Digital Equipment

Corp. Users' Society (DECUS),

St. Louis, MO

J. D. Barnes

May 1983

Composite Resin Chemistry

International Symposium on

Posterior Composite Resins,

Chapel Hill, NC

R. L. Bowen

October 1982

The Development of Composites \&

Recent Innovations in Research Labs.

New Port Harbor Academy of Dentistry,

Newport Beach, CA

R. L. Bowen

October 1982

Direct Bonding to Dentin American

Dental Association Annual Meeting,

Las Vegas, NV

R. L. Bowen

November 1982

Adhesion of Restorative Materials

to Enamel and Dentin American Academy

of Plastics Research in Dentistry,

Northwestern Dental School, Chicago, IL

R. L. Bowen

February 1983

Mechanisms in the Bonding of Resins to Dentin

AADR, Cincinnati, $\mathrm{OH}$

R. L. Bowen

March 1983

Adhesive Bonding of Various Materials to Hard Tooth Tissues

Symposium on Dental Adhesives,

$3 \mathrm{M}$, Kenora, Ontario

R. L. Bowen

June 1983 
Some New Bonding Methods

Melbourne Materials Symposium,

Victoria, Australia

R. L. Bowen

August 1983

Composites-Past, Present and Future

Kerr Division of Sybron Corp.,

Romulus, MI

R. L. Bowen

September 1983

Recently Developed Concepts in

Adhesive Bonding of Composites

to Dentin and Enamel 6 th Annual

Greater Niagara Frontier Dental Meeting,

Buffalo, NY

R. L. Bowen

September 1983

Composites--Past, Present, and

Future Kerr Division of Sybron

Corporation,

Romulus, MI

R. L. Bowen

September 1983

Properties, Structure, Modeling and Applications of Ferroelectric

and Paraelectric PVDS Polymers 1983

IEEE Int 1. Symp. on Applications of

Ferroelectrics,

Gaithersburg, MD

M. G. Broadhurst

June 1983

Physical Basis of Transducer

Properties in PVDF Int1. Symp.

on Piezoelectricity in Biomaterials

and Biomedical Devices,

Pisa, Italy

M. G. Broadhurst

June 1983

Aspects of Modeling of

Ferroelectricity in PVDF

and Related Polymers NSF/

US/Japan Workshop on

Piezoelectric Polymers,

Honolulu, Hawaj j

M. G. Broadhurst

July 1983

The Gibbs-Duhem Equation and

Calcium Phosphate Systems ASDR,

Cincinnati, $\mathrm{OH}$

W. E. Brown

March 1983

Acidic Calcium Phosphate Precurosor

in Enamel Formation Gordon Conference

on Calcium Phosphates,

Plymouth, NH

W. E. Brown

June 1983 
Heat Capacity of Phenolic Resins

Symposium on Phenolics Revisited,

75 Years Later,

Washington, DC

S. S. Chang

August 1983

Simultaneous Dissolution and

Precipitation of Calcium Phosphate

in Gels AADR,

Cincinnati, $\mathrm{OH}$

L.C. Chow

March 1983

A Procedure for Bonding to

Both Dentin and Enamel AADR,

Cincinnati, $\mathrm{OH}$

E. N. Cobb

March 1983

Phase Transitions and Ferroelectric

Polarization in a Vinylidene Fluoride-

Trifluoroethylene Copolymer

Hokaiddo University,

Sapporo, Japan

G. T. Davis

October 1982

Polarization Distribution in

Polymer Films University of Tokyo,

Tokyo, Japan

G. T. Davis

October 1982

Phase Transitions and Ferroelectric

Polarization in a Vinylidene-fluoride-

Trifluoroethylene Copolymer Institute of

Phys. \& Chem. Research,

Saitoma, Japan

G. T. Davis

October 1982

Phase Transitions and Ferroelectric

Polarization in a Vinylidene-fluoride-

Trifluoroethylene Copolymer

Nagoya, Japan

G. T. Davis

October 1982

Polarization Distribution in

Polymer Films Chung-Shan

Institute of Sci. \& Technology,

Taiwan Republic of China

G. T. Davis

October 1982

Hysteresis in Copolymers of

Vinylidene Fluoride and

Trifluoroethylene East West

Convention Center,

Honolulu, Hawai i

G. T. Davis

July 1983 
Polymeric Transduction Phenomena

and Their Role in Biomedical

Applications Intl. Symp. on

Piezoelectricity in Biomaterials

and Biomedical Devices,

Pisa, Italy

A. S. DeReggi

June 1983

Porcelain to Metal Bond Strength

Measurements as Determined by the

Four Point Bending of a Composite

Beam ASDR,

Cincinnati, $\mathrm{OH}$

W. G. de Rijk

March 1983

A Quantitative and Expedient

Test for Porcelain-to-Metal

Bond Strength, Symp. on Mechanical

Properties, Performance, and Failure

Modes of Coatings

NBS, Gaithersburg, MD

W. G. de Rijk

May 1983

Thermal and Photolytic Degradation

of PMMA University of Aston,

Birmingham, UK

B. Dickens

December 1982

The Role of Fluorescence in

the Photo-0xidation of Poly (methyl

methacrylate) John Daiton Institute,

Institute of Technology,

Manchester, UK

B. Dickens

December 1982

Degradation in Solar Cover

Plate Materials Centre

Scientifique et Technique

des Batiments,

Grenoble, France

B. Dickens

December 1982

Role of Oxygen Diffusion in the

Photodegradation of Polymethacrylates

Laboratorie de Photochemie, General Ecole

Nationale Superieure de Chimie,

Mulhouse, France

B. Dickens

December 1982

Mechanisms of Thermal and

Photo-0xidation of Polymethacrylates

Centre de Recherches sur les Macromolecules,

Strasbourg, France

B. Dickens

December 1982 
The Mechanism of Oxidation and

Role of Oxygen Diffusion in the

Photolys is of Poly(methyl)Methacrylate

Techrische Universitat,

Stutigart, West Germany

B. Dickens

December 1982

Initiation of Degradation by

Photolysis, Especially in PMMA

Hahn-Meitner Institute,

W. Berlin, West Germany

B. Dickens

December 1982

Thermal and Photodegradation of

Plates of PMMA Soc. of Plastics

Engineers, Mid-Hudson Section,

Poughkeepsie, NY

B. Dickens

March 1983

Solar Type Degradation of Plates

of PMMA Containing Monomer Rohm

and Haas Inc.,

Bristol, PA

B. Dickens

May 1983

Biological Self Assembly Modeled

as Phase Transition Phenomena

Georgetown University,

Washington, $\mathrm{DC}$

E. A. DiMarzio

October 1982

Phase Transitions in Polymers as

Models for Self Assembly in Biology

Wash. Chapter of Alpha Chi Sigma, American University,

Washington, DC

E. A. DiMarzio

October 1982

Phase Transition in Polymers;

Their Use as Models for Self Assembly

in Biology University of Virginia,

Charlottesville, VA

E. A. DiMarzio

October 1982

Phase Transitions in Polymers;

Their Use as Models for Self

Assembly University of Maryland,

College Park, MD

E. A. DiMarzio

March 1983

The Proof of the Necessity of a Low

Temperature Equilibrium Phase in Polymer

Glasses Int1. Symp. on Structure and

Bonding in Noncrystalline Solids,

Reston, VA

E. A. DiMarzio

May 1983 
Nature of the Glass Transition:

Configurational Entropy as a

Unifying Concept for Glasses

Virginia Polytechnic Institute

and State University,

Blacksburg, VA

E. A. DiMarzio

July 1983

Crystal Structures and Disorders

in Polytetrafluoroethylene

Georgia Inst. of Technology,

Atlanta, GA

R. K. Eby

October 1982

Crystal Structures and Disorders in Polytetrafluoroethylene

University of Cincinnati,

Cincinnati, $\mathrm{OH}$

R. K. Eby

October 1982

Opportunities and Needs for NDE and Accurate Service Life Prediction of Polymers Joint Seminar for ASTM

for Nondestructive Testing,

Linthicum, MD

R. K. Eby

December 1982

Overview of the Polymer Science

and Standards Division Martin

Marietta Laboratories,

Baltimore, MD

R. K. Eby

January 1983

Opportunities and Needs for Research on the Performance of Polymers 6th

Asilomar Conf. on Polymeric Materials, Pacific Grove, CA

R. K. Eby

February 1983

Structures and Transitions in

Tetrafluoroethylene Polymers

University of Utah,

Salt Lake City, UT

R. K. Eby

July 1983

Molecular Processes Affecting Mechanical

Durability of Polymers NBS Director's

Seminar Series,

Gaithersburg, MD

B. M. Fanconi

January 1983

Polymer Durability University of Utah,

Salt Lake City, UT

B. M. Fanconi

February 1983 
Chain Scission and Mechanical

Degradation of Polymers American

Physical Society,

Los Angeles, CA

B. M. Fanconi

March 1983

Thermal Degradation of Polymers

Macromolecular Section of the

Chemical Society of Vojvodina

Novi Sad, Yugoslavia

J. H. Flynn

September 1982

Prediction of Service Lifetimes of

Polymers by Thermogravimetry Macromolecular

Section of the Society of Chemistry and

Technology of Croatia,

Zagreb, Yugoslavia

J. H. Flynn

September 1982

Prediction of the Service Life of a

Polymer by Thermogravimetry Symposium

on Polymer Characterization by Thermal

Methods, 1983 ACS Middle Atlantic

Regional Meeting,

White Haven, PA

J. H. Flynn

April 1983

Application of Corrections for the Slope

of the Doyle Approximation for the Logarithm

of the Temperature Integral to the Determination

of Activation Energy by the Isoconversional Method

North American Thermal Analysis Society 12 Conf./

38 th Calorimetry Society Conf.,

Williamsburg, VA

J. H. Flynn

September 1983

The Isoconventional Method for Determination of Energy of Activation at Constant Heating Rates--Corrections for the Doyle Approximation

12th North American Thermal Analysis Society and

38th Calorimetry Conference,

Williamsburg, VA.

J. H. Flynn

September 1983

Computer Simulation of a Diffusion

Controlled Model for Caries AADR,

Cincinnati, $\mathrm{OH}$

T. M. Gregory

March 1983

Some Remarks on the History and Development of the ASTM E-37 Purity Method Symposium on Purity Determines by Thermal Analysis,

Baltimore, MD

C. M. Guttman

April 1983 
Effects of Excluded Volume and Hydrodynamic Interaction In Polymer Solutions Nagoya University,

Nagoya, Japan

c. C. Han

April 1983

Dynamic Light Scattering from Dilute and Semidilute Polymer Solutions Institute of Chemistry,

Beijing, China

C. C. Han

April 1983

Measurements of Block Chain Dimensions by

Small Angle Neutron Scattering Institute of Chemistry, Beijing, China

C. C. Han

Apri 11983

Dynamic Light Scattering of Polystyrene from

Semidilute Polymer Solutions Department of Chemistry,

Osaka University,

Osaka, Japan

C. C. Han

May 1983

Dynamic Light Scattering from Polymer

Solutions Kyoto University,

Uji, Japan,

C. C. Han

Hay 1983

Dynamic Light Scattering from Dilute Polymer Solutions

in the Intermediate q-region Kyoto University,

Kyoto, Japan

C. C. Han

June 1983

Light and Neutron Scattering from Polymers

Korean Standard Research Institute,

Daejeon, Korea,

C. C. Han

June 1983

Dynamic Light Scattering from Dilute and Semidilute Solutions Kyushyu University,

Fukuoka, Japan

C. C. Han

June 1983

Small Angle Neutron Scattering Measurements of Portions of Polymer Chains Tokyo

Institute of Technology,

iokyo, Japan

C. C. Han

June 1983

Internal Chain Expansions Measured by SANS

iokyo Metropolitan University,

Tokyo, Japan

C. C. Han

June 1983 
Polymer Diffusion and Frictional Coefficient in Semidilute Solutions Japan Fiber \& Textile Society, Kyoto, Japan

C. C. Han June 1983

Polymer Dimensions and Characterization by Light and Neutron Scattering Daicel Company, Himejo, Japan

C. C. Han June 1983

SANS Measurements of Block Chains at NBS American Chemical Society Meeting. Washington, $D C$

C. C. Han

August 1983

Review of NBS Programs on Adhesion and Adhesion Science NASA/DOD Workshop on Adhesion and Adhesives, Hyannis, MA

D. L. Hunston

October 1982

Nondestructive Evaluation of Polymer Surfaces, Interfaces, and Films via Ultrasonics Paint Res. Inst. Symp. at the Annual Mtg. of the Federation of Societies for Coating Technology, Washington, $D C$

D. L. Hunston

November 1982

Failure Behavior of Polymer Adhesives and Composites Bendix Corporation, Columbia, MD

D. L. Hunston November 1982

The Doubie Cantilever Beam Test for Interlaminar Fracture of Composites ASTM D30 Meeting, Task Group on Interlaminar Fracture Toughness, San Francisco, CA

o. L. Hunston

December 1982

Fundamentals of Adhesion Center

for Professional Advancement,

East Brunswick, NJ

D. L. Hunston

January 1983

ioughening of Polymers for Improved

Comosites and Structural Adhesives, Crevical Society of Washington. vishington, $D C$

o. L. Hunsiton

innuary 1983 
Mechanics of Adhesive Bond Failure Center for Professional Advancement, San Mateo, CA

D. L. Hunston

March 1983

Durability of Adhesive Bonds Center

for Professional Advancement,

San Mateo, CA

D. L. Hunston

March 1983

Characterization of Interlaminar

Crack Growth with the Double Cantilever

Beam Specimen NASA Workshop of Tough Composites, Hampton, VA

D. L. Hunston

May 1983

Failure Behavior of Elastomer-Modified Epoxies Sandia National Laboratories, Albuquerque, NM

D. L. Hunston

June 1983

General Rheology and its Applications

in Adhesion Workshop on Adhesion and

Adhesives, Kent State Univ.,

Kent, $\mathrm{OH}$

D. L. Hunston

June 1983

Viscoelastic Fracture of Adhesive

Joints Workshop on Adhesion and

Adhesives, Kent State Univ.,

Kent, $\mathrm{OH}$

D. L. Hunston

June 1983

Flow Fundamentals in Fluid Systems

Containing High Molecular Weight Additives

Workshop on Rheology for the Industrial Chemist, Kent State Univ.,

Kent, $\mathrm{OH}$

D. L. Hunston

June 1983

Fracture Behavior of Brittle Materials

Workshop on Rheology for the Industrial

Chemist, Kent State Univ.,

Kent, $\mathrm{OH}$

D. L. Hunston

June 1983

Effects of Formulation on the Failure

Behavior of Rubber-Modified Epoxies Gordon

Research Conference on Thermosetting Polymers, Meriden, NH

D. L. Hunston

August 1983 
Failure Behavior of Rubber-Toughened Epoxies

in Bulk, Adhesives and Composite Geometries Symp.

on Rubber Modified Thermoset Resins, American Chem. Soc.,

Washington, DC

D. L. Hunston

August 1983

Scanning Transmission Electron Microscopy

of Polyethylene Crystals Workshop on the

Electron Microscopy of Polymers, Univ. of Mass.,

Danvers, MA

F. A. Khoury

June 1983

A Water Rich Calcium Phosphate AADR,

Cincinnati, $\mathrm{OH}$

M. Mathew

March 1983

Simultaneous Measurement of Torque and

Normal Force Responses in Torsion-An Efficient

Means of Testing Constitutive Equations Ecole

Superieure de Physique et de Chimie Industrielles,

Paris, France

G. B. McKenna

October 1982

An Outlook on the Rheology of High Polymers Saclay,

France

G. B. McKenna

October 1982

Nonlinear Viscoelastic Behavior of

PMMA in Torsion Ecole de Mines,

Nancy, France

G. B. McKenna

November 1982

Effects of Large Deformations on Aged

Polymer Glasses American Society of Mechanical Engineers, Houston, TX

G. B. McKenna

June 1983

Simultaneous Measurement of Torque and

Normal Force Responses as Probes of Physical

Aging in Glassy Polymers VPI Summer Workshop

on Relaxation Effects in Disordered Systems,

Blacksburg, VA

G. B. McKenna

July 1983

Anomalies in the Physical Ageing Behavior

of PMMA 12th NATAS Annual Calorimetry Conference,

Williamsburg, VA

G. McKenna

September 1983

The Influence of Composition on the

Fusion Temperature of Experimental Dental

Porcelains Determined by Multiple Regression AADR,

Cincinnati, $\mathrm{OH}$

D. L. Menis

March 1983 
Absorption of $\mathrm{N}, \mathrm{N}$-dimethyl-p-amino-phenyl-acetic Acid on Hydroxyapatite AADR,

Cincinnati, $\mathrm{OH}$

D. N. Misra

March 1983

Relation Between Clinical Behavior and Expansion of Three Amalgams AADR,

Cincinnati, $\mathrm{OH}$

N. W. Rupp

March 1983

The Influence of the Polymer Morphology

on the Transport Properties 19th Annual

Meeting of the Society of Engineering Science,

Rolla, MO

A. Peterlin

October 1982

Relation Between Clinical Behavior and

Expansion of Three Amalgams AADR,

Cincinnati, $\mathrm{OH}$

N. W. Rupp

March 1983

Dental School, Tufts University, Boston, MA

N. W. Rupp

November 1982

Selection and Handling of Dental Materials

Egin Air Force Base, FL

N. W. Rupp

October 1982

Cavity Preparation Modifications Made

Possible by the Acid Etch Technique,

Maryland State Dental Meeting,

Baltimore, MD

N. W. Rupp

October 1982

Selection and Use of Dental Materials

Scott Air Force Base, IL

N. W. Rupp

November 1982

Impression and Die Materials Naval Dental School, Bethesda, MD

N. W. Rupp

December 1982

Esthetic Restorative Materials - New Developments

Naval Dental Center, Bethesda, MD

N. W. Rupp

February 1983

The Hazards to the Patient of Mercury in

Dental Amalgam Restorations American Association

of Dental Schools,

Cincinnati, $\mathrm{OH}$

N. W. Rupp

March 1983 
Recent Developments in Materials For

Operative Dentistry Dental School,

Georgetown University, Washington, DC

N. W. Rupp

March 1983

The Selection and Use of Dental Materials

for Removable Partial Dentures Veterans Administration,

Washington, DC

N. W. Rupp

March 1983

The World of Dental Materials

Johns Hopkins University Center for

Materials Research

Baltimore, MD

N. W. Rupp

March 1983

Sealing the Gingival Margin of

Composite Restorations AADR,

Cincinnati, $\mathrm{OH}$

N. W. Rupp

March 1983

Recent Developments in Esthetic

Restorative Materials East Central

Dental Society,

Champaign, IL

N. W. Rupp

May 1983

The Selection and Use of Dental Materials Lackland Air Force Base, FL

N. W. Rupp

May 1983

Recent Developments in Dental Materials

Office of the Surgeon General,

U.S. Air Force, Shreveport, LA

N. W. Rupp

September 1983

Selection and Manipulation of Dental

Materials Veterans Administration,

Washington, DC

N. W. Rupp

September 1983

Polymer Blends ARCO Chemical Co., Newton Square, PA

I. C. Sanchez

February 1983

Statistical Thermodynamics of Polymeric Fluids University of Maryland, College Park, MD

I. C. Sanchez

March 1983 
On the Nature of Polymer Interfaces and

Interphases National Research Council of

Canada, Ind. Materials Res. Inst.,

Montrea 1, Canada

I. C. Sanchez

April 1983

Liquids: Surface Tension, Compressibility, and

Invariants Washington Area Statistical Physics Symp.,

Gaithersburg, MD

I. C. Sanchez

April 1983

Bulk and Interface Thermodynamics of

Polymer Blends Int 1. Symp. of Phase

Relationships and Properties of

Multicomponent Polymer Systems,

Capri, Italy

I. C. Sanchez

May 1983

On the Nature of Polymer Interfaces and

Interphases G. E. Research and Development Center, Schenectady, NY

I. C. Sanchez

May 1983

On the Nature of Polymer Interfaces and Interfaces Xerox Research Laboratories, Webster, NY

I. C. Sanchez

May 1983

Center for Research on Macromolecules,

Strasbourg, France

G. A. Senich

September 1982

Inverse Phase Gas Chromatographic Studies of

Polymers Textile Institute of France,

Boulogne-Billancourt, France

G. A. Senich

September 1982

Studies of Polymer-Solvent Interactions by

Inverse Gas Chromatography: Thermodynamics and

Diffusion Eastman Kodak Company,

Kingsport, TN

G. A. Senich

October 1982

Studies of Polymer-Solvent Interactions by

Inverse Gas Chromatography: Thermodynamics and

Diffusion Polytechnic Institute of New York,

Brooklyn, NY

G. A. Senich

October 1982

Careers in Polymer Chemistry at Government

and Industrial Research Laboratories Howard University, Washington, D.C.

G. A. Senich

October 1982 
Migration from Polymers: Thermodynamics and Diffusion by Inverse Gas Chromatography E. I. du Pont de Nemours \& Company,

Wilmington, DE

G. A. Senich

April 1983

Physical Chemistry Studies of Polymers by

Inverse Gas Chromatography Washington

Chromatography Discussion Group,

Rockville, MD

G. A. Senich

May 1983

Application of X-ray Magnifier to

Microradiography of Dental Specimens--

A Preliminary Report AADR,

Cincinnati, $\mathrm{OH}$

S. Takagi

March 1983

The World of Dental Materials

Johns Hopkins University,

Baltimore, MD

J. A. Tesk

March 1983

The Need for Beryllium in Dental

Casting Alloys Ann. Session Am.

Assoc. of Dental Schools, Biomaterials Sec.

Cincinnati, $\mathrm{OH}$

J. A. Tesk

March 1983

Hydroylsis of Amorphous Calcium Phosphate

(ACP) with an Intermediate State AADR,

Cincinnati, $\mathrm{OH}$

M. S. Tung

March 1983

What Does Solid State ${ }^{13} \mathrm{C}$ NMR

Reveal About the Microstructure

of Celiulose Institute of Paper Chemistry, Appleton, WI

D. L. VanderHart

October 1982

NMR of Solids University of Florida, Gainesville, FL

D. L. VanderHart

March 1983

NMR of Solid Polymers

University of Florida,

Gainesville, FL

D. L. VanderHart

March 1983

NMR of Solid Polymers

University of California,

San Diego, CA

D. L. VanderHart

March 1983 
Studies of Microstructure in Native

Celluloses Using Solid State ${ }^{13} \mathrm{C}$ NMR Intl.

Dissolving and Specialty Pulp Conference,

Boston, MA

D. L. VanderHart

Apri1 1983

High Resolution ${ }^{13} \mathrm{C}$ NMR of Solid

Polymers Owens Corning Fiberglass,

Granville, $\mathrm{OH}$

D. L. VanderHart

Apri 1983

NMR Studies of Oriented Polymers Case

Western Reserve University,

Cleveland, $\mathrm{OH}$

D. L. VanderHart

April 1983

Native Cellulose: A Crystalline Composite

ACS National Meeting,

Washington, DC

D. L. VanderHart

August 1983

Combined ${ }^{13} \mathrm{C}$ and ${ }^{1} \mathrm{H}$ NMR Methods

for the Examination of Orientation

and Microstucture in Polymers 15th

Europhysics Conf. on Macromolecular Physics,

Hamburg, W. Germany

D. L. Vandertiart

September 1983

Investigation of Microstructure by

Solid State NMR University of Stuttgart,

Stuttgart, W. Germany

D. L. VanderHart

September 1983

13NMR Methods for the Examination of Orientation and Microstructure in

Polymers Europhysics Conference,

Hamburg, Germany

D. L. VanderHart

September 1983

Solid State NMR Studies of Cellulose Centre d'Etudes Nucleaires de Grenoble, Grenoble, France

0 . L. Vanderiart

September 1983

Low Shear Viscosity of Ultra High Molecular Weight Polycohylene Arco Chemical Company, Newtown Square, PA

H. L. Wagner

March 1983 
Applications of Fluorescence Techniques

to Polymer Systems: Polymer Compatibility \& Kyoto, Japan

F. W. Wang

May 1983

Some Studies on Chemical Durability of Polymers Ube Industries, Ltd. Hirakata Plastics Lab.,

F. W. Wang

May 1983

Applications of Fluorescence Techniques to Polymer Systems: Polymer Compatibility and Segmental Mobility Kyoto University, F. W. Wang

May 1983

Applications of Fluorescence Techniques to Polymer Systems: Polymer Compatibility \& Segmental Mobility, Osaka University,
Toyonaka, Japan F. W. Wang

May 1983

Some Studies on Chemical Durability of Polvmers University of Tokyo, Komaba, Meguroku, Tokyo, Japan

F. W. Wang

June 1983

Applications of Fluorescence Techniques to Polymer Systems: Polymer Compatibility and Segmental Mobility Research Institute of Polymers and Textiles,

Tsukuba, Japan

F. W. Wang

June 1983

Measurement of Polymer-Polymer Compatibility by Non-Radiative Energy Transfer Technique Tokyo

Woman's Christian University,
Tokyo, Japan

F. W. Wang

June 1983

Applications of Fluorescence Techniques to Polymer Systems: Additive Migration, Polymer Compatibility, and Curing Kinetics ChungShan Institute of Science and Technology,

F. W. Wang

June 1983

Applications of Fluorescence Techniques to Polymer Systems: Additive Migration, Polymer and Manufacturing Research Center Refining Petroleum Corporation,

Chia-Yi, Taiwan

F. W. Wang

June 1983 
Progress in Characterization of Macromolecules Gas Research Institute,

Evanston, IL

F. W. Wang

September 1983

Comparison of the Refractory

Metal-Noble Metal Phase Diagrams

$T^{5}-T^{10}$ Materials Research Society, Boston, MA

R. W. Waterstrat

November 1982

Idiosyncrasies of the Less Common Metals Alloy Phase Diagram Workshop,

NBS

R. M. Waterstrat

January 1983

Small Angle X-Ray Scattering of Composites

University of Hamburg,

West Germany

W. Wu

October 1982

Small Angle Neutron Scattering of

Partially Segregated Polyethylene Blends

University of Hamburg,

West Germany

W. Wu

October 1982

Small Angle Neutron Scattering of Partially Segregated Amorphous Polymeric Blends

University of Hamburg,

West Germany

W. Wu

November 1982

Wear and Degredation of Dental Composites

Free University of Berlin,

West Germany

W. Wu

November 1982

Chemical Factors Affecting Wear

Resistance of Dental Restorative

Composite Resins Royal Dental College,

Copenhagen, Denmark

W. Wu

December 1982

Random Phase Approximation to the

Paraclustering Phenomena of Polymer Blends

University of Hamburg,

West Germany

W. Wu

December 1982

Surface Breakdown and Laboratory Wear

of Dental Composites IADR,

Sydney, Australia

W. Wu

August 1983 
Small Angle Neutron Scattering Study of Polyethylene Deformation Process

ACS Conference,

Washington, DC

H. Wu

August 1983

Society of Rheology

Evanston, IL

L. J. Zapas

October 1982

The Necking of Semicrystalline Polymers

in Tension University of Minnesota,

Minneapolis, MN

L. J. Zapas

March 1983

A One Dimensional Description of the

Behavior of UHMWPE in Tension at Several

Deformations University of Minnesota,

Minneapolis, MN

L. J. Zapas

March 1983

\section{Seminars for Staff and Guests}

Piezo and Pyroelectricity of PVDF/PMMA Blends

Dr. Bernd R. Hahn

Deutsches Kunststoff Institut,

Germany

October 1982

Electrical Breakdown in P.E. and its Morphology Professor K. Yahagi

Waseda University, Tokyo, Japan

October 1982

Nonlinear Finite Element Analysis of

Radially Loaded Fiberglass Composite

Rings and Applications to Large Pressure Vessels

Professor S. G. Ladkany

Johns Hopkins University, Baltimore, MD

October 1982

Addition Polyimides

Professor (Mrs.) I. K. Varma

Indian Inst. of Technology,

New Delhi, India

November 1982

The Preparation and Properties of

Ultra High Modulus Polyolefins

Professor I. M. Ward

University of Leeds, Leeds, England

November 1982

AC Partial Discharge and Surface

Effects in Polymer Films

Professor W. J. Sarjeant

State University of New York at Buffalo, Amherst, NY November 1982 
Morphology and Toughness Comparison of

Two Anine Cured Rubber Epoxies

Dr. Stephanie C. Kunz

Sandia Laboratories, Albuquerque, NM

November 1982

Polymer Surfactant Interactions

Dr. Pak S. Leung

Union Carbide Corp., Terrytown, NY

December 1982

The Silver Electrode:

Antibacterial Effects of Ostagenesis

Dr. Joseph Spadaro

State University of New York, Syracuse, NY

December 1982

Interfacial Structures of Composite Materials

Professor H. Ishida

Case Western Reserve University, $\mathrm{Clevel}$ and, $\mathrm{OH}$

December 1982

Light Reflection by Dental Enamel

Dr. Roelf A. J. Groenhuis

NIDR, NIH, Bethesda, MD

December 1982

Property Relationships in Miscible Polymers Blends

Professor Donald R. Paul

The University of Texas, Austin, TX

December 1982

Structure and Properties of Polydiacetylenes

Produced from Polyfunctional Diacelytene Monomers

Professor J. B. Lando

Case western Reserve University, Cleveland, $\mathrm{OH}$

December 1982

Associative Thicknesses

Dr. Barry Bauer

45 Broad St., Summit, NJ

January 1983 .

Etched Cast Restoration: What We Don't Know

Dr. Van P. Thompson

University of Maryland Dental School, Baltimore, MD

January 1983

Conformational and Dynamic Properties of Olefin/

SO, Polymers in Solution

Professor Walter H. Stockmayer

Dartmouth College, Hanover, $\mathrm{NH}$

February 1983

Recent Measurement of Polymer Interaction

Parameter by Scattering Methods

Professor Richard S. Stein

University of Massachusetts, Amherst, MA

February 1983

Injection Moulding of High Strength Polyethylene Dr. R. K. Bayer

University of Kassel, West Germany

February 1983 
Entropy Correlation Theory and Diffusion

Measurements for Oriented Polymers

Dr. R. E. Barker, Jr.

University of Virginia, Charlottesville, $V A$

February 1983

Characterization of Linear and Branched

Polyethylene by Laser Light Scattering

Professor Ben Chu

State University of New York at Stonybrook

February 1983

Diffusion Controlled Growth and Fractal Structure

Dr. Paul Meakin

DuPont Experimental Station, Wilmington, $D E$

February 1983

Cold-Drawing of Polymers

Professor Bernard Coleman

Carnegie-Mellon Univ., Pittsburgh, PA

March 1983

Nonlinear Viscoelasticity of Molten Plastics

Professor Johm M. Dealy

McGill University, Montreal, Canada

March 1983

Osteoinductive Materials from Bone Matrix

Dr. Karl Schmitd

University of Tuebingen, Tuebingen,W. Germany

March 1983

Long Chain Polymers as Substrates for

Biomedical and Organometallic Reactions

Dr. Harry Allcock

Penn State University, University Park, PA

March 1983

Low Frequency Raman Scattering from

Vitreous and Molten $\mathrm{B}_{2} \mathrm{O}_{3}$

Professor George Walrafen

Howard University, Washington, DC

March 1983

Synthetic Techniques for Living Polymers

Dr. Barry Bauer

45 Broad St., Summit, NJ

April 1983

Fluoridation of Enamel and Microleakage

Around Dental Amalgams

Dr Hugo Retif

University of Alabama, Birmingham, AL

April 1983

Influence of Combined Linear \& Rotating

Motion on the Friction \& Wear of Polymers

Dr. Brian Briscoe

Imperial College, London, England

April 1983 
Xray Study of the Structure of

Liquid Crystalline Polyesters

Dr. John Blackwell

Case Western Reserve University, Cleveland, $\mathrm{OH}$

April 1983

Descriptions of Diffusion of Small

Molecules in Polymer Blends

Professor Julio Ottino

University of Massachusetts, Amherst, MA

April 1983

New Polymer Structures and Their Properties

Professor Otto Vogl

Polytechnic Inst. of New York, Brooklyn, NY

April 1983

The Morphology of MDI/BDO Based Polyurethanes

Dr. Robert Briber

University of Massachusetts, Amherst, MA

April 1983

Biocompatibility of Dental Casting Alloys

Dr. Heiner

Weber University of Tuebingen, Tuebingen, W. Germany

May 1983

Radiation Chemistry of Polyethylene

and Crystalline Alkanes

Professor Joseph Silverman

University of Maryland, College Park, MD

May 1983

New Aspects of Electrical Conduction in Polymers

Professor Frank Karasz

University of Massachusetts, Amherst, MA

May 1983

Configuration of Immiscible fluids with

Different Viscosities

Professor D. D. Joseph

University of Minnesota, Minneapolis, MN

May 1983

Endurance of Electrical Insulating Materials

for Combined Thermal Electrical Stress

Professor Luciano Simini

University of Bologna, Bologna, Italy

May 1983

Infrared Intensities: Theory and Applications

Dr. Giuseppe Zerbi

Institute of Macromolecular Chemistry, Milan, Italy

May 1983

Functional Representation of the

Castability of Dental Alloys

Dr. Susumo Hirano

Tsurumi University, Yokohania, Japan

May 1983 
Curing Kinetics and Morphology of

High Temperature and Rubber Toughened Epoxy

Dr. D. Hong

Jet Propulsion Laboratory, Pasadena, CA

May 1983

Corrosion Products of Dental Amalgams

Dr. Sally Marshall

Northwestern University, Chicago, IL

June 1983

Fourier Cross-Correlation Analysis of Impedance

Spectra of Solid State Electrolytic Cells

Dr. Alan D. Franklin NBS, Retired

June 1983

Thermodynamics, Kinetics, Morphology and Chemistry

of Calcium Phosphate Crystal Growth

Dr. E. D. Eanes

NIDR, NIH, Bethesda, MD

June 1983

Calcium Phosphate Inhibitors in Blood

Dr J. L. Meyer

NIDR, NIH, Bethesda, MD

June 1983

Hybrid Materials.and Their Applications

Dr. Hiroyuki Sasabe

Institute of Physical \& Chemical Research, Saitama, Japan

June 1983

Photochemical Investigations of Polymers:

Charge Transfer Complexes in Polyolefins, Energy

Migration, Photoyellowing of Epoxy Resins

Dr. N. S. Allen Manchester, United Kingdom

June 1983

LCST Behavior in Polymer Blends: Directional

Specific Interactions and Compressibility

Dr. Gerrit ten

Brinke University of Massachusetts, Amherst, MA

June 1983

Diffraction Studies of Craze Structures

Professor Hugh Brown

Case Western Reserve University, Cleveland, $\mathrm{OH}$

June 1983

Scattering from a Polymer Solution and a

Mixture of Polymers at an Arbitrary Concentration

Dr. Henri Benoit

Centre de Recherches sur les Macromolecules, Strasbourg, France

June 1983

Scattering from Copolymer Solutions

Dr. Henri Benoit

Centre de Recherches sur les Macromolecules, Strasbourg, France

June 1983 
Piezoelectric and Ferroelectric Properties of

Vinylidenefluoride, Trifluoroethylene Copolymer

and its Application to UTtrasonic Transducers

Dr. Hirogi Ohigashi

Toray Industries, Kamakura, Japan

June 1983

Some Recent SANS Results on

Poly(dimethylsiloxane) Networks

Dr. Henri Benoit

Centre de Recherches sur les Macromolecules, Strasbourg, France

June 1983

Air Force Ordered Polymer Research Program

Dr. Wade Adams

Wright Patterson AFB, Dayton, $\mathrm{OH}$

July 1983

The Microstructure and Deformation Behavior of Highly Oriented Polyethylene Films

Dr. Wade Adams

Wright Patterson AFB, Dayton, $\mathrm{OH}$

July 1983

Mechanisms of Network Formation

by Radical Copolymerization

Dr. Girard Hild

Centre de Recherches sur les Macromolecules, Strasbourg, France

July 1983

Crazing in Semi Crystaliine Isotactic Polystryrene

Professor David T. Grubb

Cornell University, Ithaca, NY

July 1983

Theory of Critical Micelle Concentrations

in Block Copolymer Systems

Dr. L. Leibler

CNRS, Strasbourg, France

JuTy 1983

Brownian Motions in Polymer Solutions and Meit

Dr. D. Richter

Institut fur Festkorperforschung, Julich, Germany

JuTy 1983

Structural Studies on Conducting Polymers

by $x$-ray and Neutron Diffraction

Dr. C. Riekel

University of Hamburg, West Germany

August 1983

Molecular Studies on Rheology and Microphase

Separation of Block Polymers

Professor Takeji

Hashimoto Kyoto University, Kyoto, Japan

August 1983

Spinodal Decomposition of Polymer Blends

Professor Takeji Hashimoto

Kyoto University, Kyoto, Japan

August 1983 
Structural Studies on Gels from

Isotactic Polystyrene

Professor Edward Atkins

Univeristy of Bristol, Bristol, England

August 1983

Overview of Theories for the Adsorption

of Homodisperse or Polydisperse Polymers

Dr. Gerard Fleer

DeBreyen 6 , Wageningen, The Netherlands

August 1983

NMR Polymers

Professor R. Kitamaru

Institute for Chemical Research, Kyoto University, Japan

August 1983

Photoinitiation of Acrylic Resins

Or. C. Decker

Laboratory of General Photochemistry Mulhouse, France

August 1983

Solid State Reaction and Its Use for the Syntheses of Novel

Organic Compounds, Conducting Polymers and Photopolymers

Dr. Hachiro Nakanishi

Research Inst. for Polymers and Textile, Ibaraki, Japan

September 1983

Time-Dependent Properties of Statically Deformed

Glassy Polymers: Dynamic Modulus and Gas Transport

Dr. Thor L. Smith

IBM Research Laboratory, San Jose, CA

September 1983

Kinetics of Decomposition and Crystallization

in Mixtures of PE and POM

Dr. B. J. Jungnickel

Deutsches KunststoffInstitut, Darmstadt, Germany

September 1983

A Multiparameter Model for Structural Recovery in

Polymer Glasses and its Implications for their

Physical Properties

Professor A. J. Kovacs

Centre de Recherches sur les Macromolecules,Strasbourg, France

September 1983

Xray Studies of the Kinetics of Crystallization

and Recrystallization of Different Polymers

Dr. H. G. Zachmann University of Hamburg, Hamburg, West Germany

September 1983

The Modeling of Crystal Growth; Roughness of

Growth Faces and Nature of the Interfaces

Professor David Sadler

University of Bristol, Bristol, England

September 1983

Recent Neutron Scattering Experiments at JulichStructure, Conformation and Diffusion of Polymers

Dr. M. Stamm

Institut fur Festkorperforschung, Julich, Germany

September 1983 
Rutherford Backscattering and NBS Facility

Dr. Hans P. R. Frederikse

Inorganic Materials Division, NBS

September 1983

Enhancement of Polymer Miscibility

Through Specific Interaction

Dr. T. K. Kwei

Industrial Technology Research Institute, Taiwan

September 1983

\section{Technical and Professional Committee Participation and Leadership}

Academy of Operative Dentistry

N. W. Rupp, Councilor

American Academy of Gold Foil Operators

N. W. Rupp, Councilor

American Association for Dental Research/International Association

for Dental Research

R. L. Bowen

Member: Dental Materials Group

Wilmer Souder Award Committee

J. M. Antonucci

Member: Dental Materials Group

G. M. Brauer

Member: Dental Materials Group

J. M. Cassel

Member: Dental Materials Group

J. E. McKinney

Member: Dental Materials Group

W. G. deRijk

Member: Dental Materials Group

Student Research Committee

G. C. Paffenbarger

Member: Dental Materials Group

N. W. Rupp

Member: Dental Materials Group

W. Wu

Member: Dental Materials Group

J. A. Tesk

Member: Dental Materials Group

Group Representative: Executive Committee

W. E. Brown

Member: Biological Mineralization Research Award Committee

Mineralized Tissue Group, Councilor

L. C. Chow

Member: Mineralized Tissue Group

Nominating Committee

Teller 
T. M. Gregory

Member: Mineralized Tissue Group

M. Mathew

Member: Mineralized Tissue Group

S. Takagi

Member: Mineralized Tissue Group

M. S. Tung

Member: Mineralized Tissue Group

American Association of Dental Research/Washington Section

J. A. Tesk

Secretary-Treasurer

American Chemical Society

J. M. Antonucci

Member: Board of Managers

Advertising Editor

G. M. Brauer

Member: Meetings and Expositions Committee

Councilor

American Dental Association

R. L. Bowen

Member: Biomedical Research Support Grant Committee

W. E. Brown

Member: Biomedical Research Support Grant Committee

American Dental Association Special Committee on the Future of Dentistry

R. L. Bowen

Member: Research Working Group

American Dental Association Specifications Committee

W. E. deRijk

Member: Subcommittee on Waxes (Specifications 4 and 24)

American Institute of Mining, Metallurgical, \& Petroleum Engineers

R. M. Waterstrat

Member: Committee on Alloy Phases

American Institute of Physics

R. K. Eby

Chapter Editor, Physics Handbook

E. A. Kearsley Member: Committee for Public Policy

American National Standards Committee, MD156, Dental Materials, Instruments, and Equipment

R. L. Bowen

Member: Subcommittee on Biological Evaluation of Dental Materials, Secretary

G. M. Brauer

Member: Government Representative

Denture Resins, Chairman

Z-E Type Material 
J. M. Cassel

Member: C054, Medical Devices Standards Management

Board, Executive Committee

N. W. Rupp

Member: Alloy for Dental Amalgams, Chairman

Dispensers for Alloys and Mercury, Chairman

Mercury for Dental Amalgam, Chairman

Mechanical Amalgamators, Chairman

J. A. Tesk

Member: Base Metal Alloys

C054, Medical Devices Standards Management Board, Executive Committee

Dental Implants, Secretary

Dental Materials, Instruments, \& Equipment, Chairman

Porcelain/Alloy Systems

USA TAG for ISO TC106, Chairman

American Physical Society

Division of High Polymer Physics

F. A. Khoury

Member: Committee on History of DHPP

I. C. Sanchez

Member: Oivision of High Polymer Physics, Chairman, 1983-84

Selection Committee, APS High Polymer Physics

Prize, 1983-84

American Society of Mechanical Engineers

R. W. Penn

Member: Reinforced Thermoset Plastic Corrosion Resistant Equipment

American Society for Testing and Materials

Committee on Research and Technical Planning

E. Passaglia

Member: Subcommittee on Research Chairman

D-2: Petroleum Products and Lubricants

L. J. Zapas

Member: D2.07 Flow Properties

D2.07A Newtonian Viscometry

D-11: Rubber and Rubber--Like Materials

E. A. Kearsley

Member

G. B. McKenna

Member

D-20: Plastics

R. K. Eby, Chairman .

Member: D20.01: Executive Subcommittee

J. D. Barnes

Member: D20.12: Olefin Plastics

D20.13: Statistics, Chairman

D20.19: Film and Sheeting

D20.70: Analytical Methods

WG. 07: Gas Permeability 
H. L. Wagner

Member:

WG.02 Liquid Size Exclusion Chromatography

WG.05 Molecular Weight Parameters, Chairman

J. H. Flynn

Member: - 020.30: Thermal Properties

30.7 Fundamental Thermal Methods, Chairman

D-30: High Modulus Fibers and Their Composites

D. L. Hunston

Member

E-9: Fatique

G. B. McKenna

Member

R. W. Penn

Member

E-37: Thermal Measurements

S. S. Chang

Member: E37.01: Test Methods and Recommended Practices

E37.05: Thermophysical Properties

J. H. Flynn

Member: E37.03: Nomenclature and Definitions, Chairman

E37.90: Executive Subcommittee

E-45: Geothermal Energy and Resources

E. A. Kearsley

Member: E45.02: Materials

F-2: Flexible Barrier Materials

J. D. Barnes

Member: F02.30: Test Methods

F02.93: Statistical Matters, Chairman

WG.30.2: Barrier Methods, Chairman

F-4: Medical and Surgical Materials, and Devices

G. M. Brauer

Member: Executive Committee

F04.20: Resources

WG.01: Polymeric Materials, Chairman

J. M. Cassel

Member: F04.20: Resources

R.K. Eby

Member

G. B. McKenna

Member

Biomedical Research Support Grant

R. L. Bowen

Member: 1982-1983 Review Committee

W. E. Brown

Member: 1982-1983 Review Committee

Applications of Ferroelectrics (ISAF), Gaithersburg, MD, 1983 IEEE Inti. Symp.

M. G. Broadhurst

Member: Program Committee 
Case Western Reserve University, Materials Research Laboratory

Advisory Board

R. K. Eby

Member

Chemical Society of Washington

J. M. Antonucci

Member: Awards Committee

G. M. Brauer

Chairman, Councilors Committee

Award Committee

B. F. Howe 11

President: 1983

G. A. Senich

Chairman: Budget Committee

DECUS (Digital Equipment Users' Society)

Washington Area MIN-LSI Users'

J. D. Barnes

Chairman

Eleventh NATAS Conference

J. H. Flynn

Symposium on Kinetics of Chemical \& Physical Processes, Chairman

Geis Foundation for the Advancement of Dentistry

G. C. Paffenbarger

President

Member: Award Committee for Dental Editorial, Chairman

Award Committee for Oral \& Maxillofacial Surgery,

Chairman

Award Committee for Periodomtology, Chairman

Government Interagency Panel on Adhesive Bonding Research

D. L. Hunston

Member

International Confederation for Thermal Analysis

J. H. Flyrn

Member: Nomenclature Committee

Working Party on Symbols

International Council on Alloy Phase Diagrams

R. M. Waterstrat

Member

International Electrotechnical Commission

TC046, Cables, Wires, \& Waveguides for Telecommunication

Equipment

L. J. Zapas

Member: WG.03: Measuring Methods of Polyethylene

TC061, Plastics

L. E. Smith

Member

International Standards Organization

TC106, Dental Materials and Devices

G. M. Brauer

Member: Task Force on Color Stability 
N. W. Rupp

Member: Restorative Materials

WG.01: U. S. Delegation, Chairman

TG7: Alloys for Dental Amalgams, Chairman

WG.06: Amalgamation Devices \& Accessories, Chairman

J. A. Tesk, USA Delegation, Chairman

TC150, Implants for Surgery, USTAG

J. M. Cassel, Chairman

TC150/SC2, Cardiovascular Implants for Surgery, USTAG

J. M. Cassel, NBS Representative

International Union of Pure and Applied Chemistry

J. H. Flynn

Member: 4. Macromolecular Division

4.2 Polymer Characterization and Properties

4.2. 4 Working Party on Thermal Properties of Polymers

Macromolecules

E. A. DiMarzio

Member: Editorial Advisory Board

Molecular and Cellular Thermobiology (Journal)

B. Dickens

Member: Editorial Board

National Bureau of Standards Advisory Panel for Tariffs on

Electron Microscopes Under Florence Agreement

F. A. Khoury, Member

National Bureau of Standards Committee on University of Maryland

Instructional Television System

E. A. DiMarzio, Member

National Bureau of Standards Research Advisory Panel

E. A. DiMarzio, Member

National Bureau of Standards Sigma $X_{i}$

B. F. Howe 11, President, 1982

Member: Executive Committee, 1983

National Center for Small Angle Scattering

C. C. Han

Member: Users Committee

National Health and Medical Research Council of Australia

G. C. Paffenbarger, Consultant

National Institute of Dental Research

R. L. Bowen

Member: Long Range Research Planning \& State-of-the-Science

on Composites, Consultant

Panel on Restorative Materials Program

Review Conmittee for ADA

National Institutes of Health

L. C. Chow

Member: Oral Biology and Medicine Study Section 
National Research Council

Panel on Polymer Science \& Engineering

R. K. Eby, Member

Committee on Room Temperature Curing Resin Systems

B. M. Fanconi, Member

Panel on Civil/Navy Ocean Engineering

Task Group on Materials and Structure

Nondestructive Testing of NRC

R. K. Eby, Member

North American Thermal Analys is Society

J. H. Flynn

International Confederation for Thermal Analysis Councilor

Member: Awards Committee

Executive Committee

Ohio Dental Association

G. C. Paffenbarger

Member: Callahan Memorial Committee

Piezoelectricity in Biomaterials and Biomedical Devices, Intl. Symposium,

Pisa, Italy

M. G. Broadhurst

Member: Program Committee

Publication Committee

Polymer (Journal)

R. K. Eby, U. S. Associate Editor

Polymer Degradation and Stablility (Journal)

J. H. Flynn, Editorial Board Member

Polymer Physics Gordon Conference

E. A. DiMarzio, Chairman

Smithsonian Institution

American Academy of the History of Dentistry

Advisory Committe

G. C. Paffenbarger

Society of Plastics Engineers

R. K. Eby

Member: Selection Committee for SPE International

Award for Research, Chairman

G. B. McKenna

Member: Technical Publications

Society of Rheology

D. L. Hunston

Member: Committee on Education

Member: Site Selection Committee

J. E. McKinney, Member

E. A. Kearsley

Member: Executive Committee

G. B. McKenna

Member: Committee on Education 
Symposium on Structure and Bonding in Noncrystalline Solids

E. A. DiMarzio, Committee member

Technical Meeting of Digital Equipment Users' Society of "Database

Management in Small Systems"

J. D. Barnes, Chairman

Third Conference on Fractals in the Physical Sciences

R. J. Rubin

Member: Organizing Committee

Thermochimica Acta (Journa?)

J. H. Flynn

Member: Editorial Board

Twelfth North American Thermal Analysis Society, 38th Calormetry Society Conf.

J. H. Flynn

Session Chairman

U.S. Air Force

National Civilian Consultant to the Surgeon General

N. W. Rupp

U.S. Army

Medical R\&D Advisory Committee

G. C. Paffenbarger

US/Yugoslav Science and Technology Program

Physico-Chemical Aspects of Urinary Stone Formation

W. E. Brown, Monitor

Thermal Degradation of Polymers

J. H. Flynn, Monitor

University of Virginia, Department of Materials Science

G. C. Paffenbarger, Consultant

University of Massachusetts Advisory Board of Polymer Research

Institute

R. K. Eby, Member

Washington Academy of Sciences

B. F. Howell

Member: Executive Committee

Washington Area MINC Users Group

J. D. Barnes, Chaiman

Washington Philosophical Society

B. F. Howell

Member: Executive Committee (Member-at-large)

Younger Chemists Committee

G. A. Senich

Member: Activities Committee

\section{Publications}

Bascom, W. D.; Hunston, D. L. The fracture of epoxy and elastomermodified epoxy polymers, chapter 14 in Adhesion 6 . Allen, K. W.

ed. London, England: Applied Science Publishers; 1982. 
Bowen, R. L. ; Cobb, E. N.; Rapson, J. E. Adhesive bonding of various materials to hard tooth tissues: improvement in bond strength to dentin. J. Dent. Res. 61(9): 1070; 1982.

Bowen, R. L.; Cobb, E. N.; Setz, L. E. Adhesive bonding to dentin and ename 1. Dentistry 82: 11 ; 1982.

Bur, A. J. Resistivity of ultra drawn polyvinylidene fluoride. 1982 Annual Report, Conference Electrical Insulation and Dielectric Phenomena; 156.

Bur, A. J. Dielectric loss spectra of polymers at microwave frequencies. Nat. Bur. Stand. (U.S.) NBSIR 82-2603; December 1982.

Chen, T. M. ; Brauer, G. M. Solvent effects on bonding organo-silane to silica surfaces. J. Dent. Res. 61: 1493; 1982.

Chow, L. C. ; Brown, W. E. An approach to remineralization via saliva. Proc. of Third Annual Conference on Foods, Nutrition and Dental Health, October 1979, Vol. 2 (Chicago, IL 1982).

Davis, G. T. Phase transition and ferroelectric polarization in a vinylidene fluoride--trifluoroethylene copolymer. Polymer Preprints, 31(5): 937 ; 1982.

Deh1, R. E. On the characterization of porosity in PTFE-carbon composite implant materials by mercury porosimetry. J. Biomed. Mater. Res. 16: 715; 1982.

Dermann, K.; Rupp, N. W.; Brauer, G. M. Effect of hydrophilic diluents on the properties of cured composites. J. Dent. Res. 61(11): 1250; 1982.

DiMarzio, E. A. ; Guttman, C. M. Polymer crystallization: proper accounting of a wider class of paths to crystaliization-variation on a theme of point. J. App1. Phys. 53: 6581; 1982.

Eby, R. K.; Davis, G. T.; Wang, F. W.; Broadhurst, M. G.; Fanconi, B. M. ; Sanchez, I. C.; Tesk, J. A. Polymer science and standards division. Kobunshi 31(11): 1026; 1982.

Han, C. C.; Amis, E. J. Cooperative and seif diffusion of polymers by dynamic scattering in semidilute solutions. Polymer 23: 1403; 1982.

Matsushita, Y.; Furakashi, H.; Choshi, H.; Noda, I.; Nagasawa, M; Fujimoto, T.; Han, C. C. Polymer Journal (Japan) 14: 489; 1982.

McKinney, J. E.; Wu, W. Relationship between surface damage and wear of dental restorative composites. J. Dental Res. 61(9): 1083; 1982.

Mjor, I. A.; Hensten-Petersen, A.; Bowen, R. L. Biological assessments of experimental cavity cleansers: correlation between in vitro and in vivo studies. J. Dent. Res. 61(8): 967; 1982.

Paffenbarger, G. C. ; Rupp, N. W. ; Coyne, M. P. Dimensional changes of four amalgams after five years of storage in air at 60,37, and $23 \mathrm{C}$. J. Dent. Res. 61(12): 1427; 1982.

Paffenbarger, G. C.; Rupp, N. W.; Patel, P. R. Dimensional changes of a high-tin content amalgam. J. Dent. Res. 61(12): 1423; 1982.

Peterlin, A. Accordion type laser-raman scattering by polymers. J. Polym. Sci. (Poly. Phys. Ed.): 20: 2329; 1982. 
Senich, G. A. A review of the migration of food-contact organotin stabilizers from poly(vinyl chloride). Polymer 23: 1385; 1982.

Takagi, S; Mathew, M.; Brown, W. E. The structure of sodium strontium phosphate nonahydrate. Acta Cryst. B38: 1408; 1982.

Wang, F. W. ; Howell, B. F. Fluorescence measurement of antioxidant migration from low-density polyethylene into 1-propanol. Proceedings of the ACS organic coatings and applied polymer science symposium; 47: 41; 1982.

Wu, W.; Mckinney, J. E. Influence of chemicals on wear of dental composites. J. Dental Res. 61(10): 1180; 1982.

Amis, E. J.; Glinka, G. J.; Han, C. C.; Hasegawa, H.; Hashimoto, T.; Lodge, T. P.; Matsushita, Y. SANS measurements of block chains at NBS. ACS Polymer Preprint 23(2): 1983.

Antonucci, J. M.; Debelius, E. Extent of polymerization of dental resins by differential scanning calorimetry. J. Dent. Res. 62(2): 121; 1983.

Barnes, J. D. Standard reference materials for gas transmission measurements in "industrial gas separations". ACS Symposium Series No. 223, T. E. Whyte, Jr., C. M. Yon, and E. H. Wagener Eds., American Chemical Society, Washington, DC 1983.

Bowen, R. L.; Nemoto, K.; Rapson, J. E. Adhesive bonding of various materials to hard tooth tissues; forces developing in composite materials during hardening. JADA 106: 475; 1983.

Brauer, G. M.; Stansbury, J. W.; Argentar, H. Development of high-strength, acrylic resin-compatible adhesive cements. J. Dent. Res. 62: 366; 1983.

Brown, W. E.; Chow, L. C. Surface equilibria of sparingly soluble crystals. Colloids and. Surfaces 7: 67; 1983.

Brown, D. W.; Lowry, R. E.; Smith, L. E. Prediction of the long term stability of polyester based recording media, Nat. Bur. Stand. (U.S.) NBSIR 83-2750; July 1983

Clark, E. S.; Eby, R. K.; Piermarini, G. J.; Block, S. The crystal structure of polytetrafluoroethylene homo and copolymers in the high pressure phase. Polymer Preprints, ACS Meeting, Washington, DC; August 1983.

Chang, S. S.; Maurey, J. M.; Pummer, W. J. Solubilities of two n-alkanes in various solvents. J. Chem. Eng. Data 28: 187; 1983.

Crissman, J. M.; Zapas, L. J.; Khoury, F. A. Relationship between morphology and mechanical properties of ultra high molecular weight polyethylene,

Third Annual report to the FDA, Bureau of Medical Devices, NBSIR 83-2696.

Crissman, J. M.; Zapas, L. J.; Mechanical behavior of isotactic polypropylene subjected to various strain histories in uniaxial extension. Polymer $24: 351 ; 1983$.

Crissman, J. M. A new test method for determining environmental stress-crack resistance of ethylene based plastics, Testing and evaluation ASTM, 11(4): 223; 1983.

DeCandia, F. D.; Perullo, A. Vittoria, V.; Peterlin, A. Mechanical and transport properties of drawn low pressure low density polyethylene. J. App 1. Poly.

Sci. 28: 1815; 1983.

Deh1, R. E. Characterization of porosity in porous polymeric implant materials. Nat. Bur. Stand. (U.S.) NBSIR 83-2645 (FDA); February 1983. 
Dickens, B.; Flynn, J. H. Thermogravimetry applied to polymer degradation kinetics. Chapter 12 in Polymer Characterization, C. D. Craver, Ed., Adv. in Chem. Series 203, ACS; Washington, DC; $209 ; 1983$.

Eby, R. K.; Davis, G. T.; Wang, F. W.; Broadhurst, M. G. ; Fanconi, B. M.; Sanchez, I. C.; Tesk, J. A. Polymer science and standards division. Polym. News 9(4): 81; 1983.

Eby, R. K. ; Broadhurst, M. G. ; Davis, G. T.; Wang, F. W.; Fanconi, B. M; Tesk, J. A.; Sanchez, I. C. Polymer science and standards division 1982. Nat. Bur. Stand. (U.S.) NBSIR 82-2607; 1983 March.

Farmer, B. L.; Eby, R. K. Molecular packing in $15 / 7$ "hexagonal" polytetrafluoroethylene. Proc. ACS Meeting, Polymer Preprints, Washington, DC; August 1983.

Fanconi, B. M. Molecular processes affecting mechanical failure of polymeric materials. Nat. Bur. Stand. (U.S.) NBSIR 83-2682; June 1983.

Flynn, J. H. The isoconversional method for determination of energy of activation at constant heating rates--corrections for the doyle approximation. J. Therm. Anal. 27; 92; 1983.

Fraker, A. C.; Rutt, A. W. ; Bundy, K. J.; Smith, J. D.; Penn, R. W.; Van Orden, A.C. Studies of interface bondings on implant alloys, Nat. Bur. Stand. (U.S.) NBSIR 83-2736.

Hansen, R. J.; Hunston, D. L. Effects of fluid viscosity and viscoelasticity on flow-generated waves on a compliant surface. 131: 161; 1983.

Hunston, D. L. Relationship between mechanical properties and performance of inks as the basic for quality control techniques. Nat. Bur. Stand. (U.S.) NBSIR 83-2691; 1983.

Hunston, D. L. ; Rushford, J. L. ; Newitt, W. R.; Vaudreuil, B. A. Rheology of cure for intaglio printing inks. Organic Coatings and Plastics Prep. 47: $513 ; 1983$.

Hunston, D. L. ; Wang, S. S.; Kinloch, A. J. Characterization of fracture behavior of adhesive joints. Organic coatings and plastics Prep. 47: 408 ; 1983.

Hunston, D. L. Cure monitoring of thermosetting polymers by an ultrasonic technique. Thompson, D. 0 ., ed. Review of Progress in Quantitative Nondestructive Evaluation; New York, NY: Plenum Publ. Co.: 1711; 1983.

Hunston, D. L. ; Rushford, J. L; Newitt, W. R.; Vaudreuil, B. A. Rheology of Cure for Intaglio Printing Inks, chapter 10 in Chemorheology in Thermosetting Polymers. May, C., ed.; ACS Symp. Ser. 227; Washington, DC, 1983.

Kranbueh1, D. E.; Verdier, P. H. Interpretation of quasi-elastic light scattering data for flexible chains: model dependence. Polymer, 24: 383; 1983.

LeGeros, R. Z.; Tung, M. S. Chemical stability of $\mathrm{CO}_{3}+\mathrm{F}$ - containing aptites. Carries Res. 17:419; 1983.

Mabie, C. P.; Menis, D. L; Whitenton, E. P.; Trout, R. L.; Metherate, R. S.; Ferry, C. H. Gel-route preparation of low fusing dental porcelain frit. J. Biomed. Mater. Res. 17(4): 691; 1983.

McKenna, G. B.; Kovacs, A. J. Anomalies in the physical ageing behavior of PMMA. Proc. 12th NATAS/38th Calorimetry Conf., Williamsburg, VA; 1983. 
McKenna, G. B.; Kovacs, A. J. Anomalies in the physical ageing behavior of PMMA, Polymer Preprints; 1983.

Metz, P. D.; Teoh, H.; VanderHart, D. L.; Wilhelm, W. G. Evaluation of polyacrylonitrile as a potential organic polymer photovoltaic material. Polymers for Solar Energy Utilization, ACS Symposium Series 220: 421; 1983.

01 sen, R. B.; Hicks, J. C. ; Broadhurst, M. G.; Davis, G. T. Temperature dependent hysteresis study in polyvinylidene fluoride; Appl. Phys. Lett. 43:127; 1983.

Perry, B. W.; Doumas, B. T.; Bayse, D. D.; Butler, T.; Cohen, A. ;

Fellow, W.; Garber, C. C.; Howell, B. F.; Koch, T.; Krishnamurthy, S.; Louderback, A. ; McComb, R. B. ; Miller, D.; Miller, R. R.; Rand, R. W.; Schaffer, R. A candidate reference method for determination of bilirubin in serum. Test for Transferability. Clin. Chem. 29: 297; 1983.

Peterlin, A. Polymer morphology \& mechanics: the strength and stiffness of polymers. New York, NY: Marcel Dekker, Inc.; 97; 1983.

Peterlin, A. Transport of small molecules in polymers. chapter in controlled drug delivery, Bruck, S. ed. CRC Press I; 15; 1983.

Peterlin, A; Mechanical and transport properties of drawn semicrystalline polymers. Chapter in the book The Strength and Stiffness of Polymers,

Zachariades, A. E.; Porter, R. S. eds. M. Dekker, New York 97; 1983.

Phillips, J. C. Mechanical relaxation of liner materials in acetic acid, Nat. Bur. Stand. (U.S.) NBSIR 82-2615 1983.

Rubin, R. J.; Weiss, G. H. Limiting thickness of an adsorbed polymer chain random flight model. J. Chem. Phys. 78: 2039; 1983.

Rubin, R. J. Span of an adsorbed chain in a lattice random walk model. But1. Am. Phys. Soc. 28(3): 473; 1983.

Sanchez, I. C.; Poser, C. I. Surface thermodynamics of liquid polymers: theory. Physicochemical aspects of polymer surfaces, Vol. I, K. L. Mittal, ed., Plenum Press, New York; 173; 1983.

Sanchez, I. C. Liquids: surface tension, compressibility, and invariants. J. Chem. Phys. 79: 405; 1983.

Sanchez, I. C. Bulk and interface thermodynamics of polymer alloys. Ann. Rev. Mat1. Sci. 13: 1983.

Senich, G. A.; Florin, R. E. Radiation curing of inks and coatings. Nat. Bur. Stand. (U.S.) NBSIR 83-2722 1983.

Takagi, S.; Mathew, M.; Brown, W. E. A glaserite-type structure; calcium

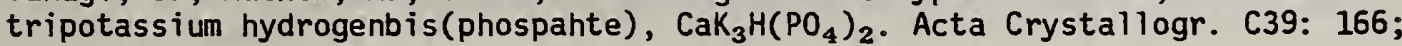
1983.

Tesk, J. A. ; Hinman, R. W.; Widera, G. E; Holmes, A. D. ; Cassel, J. M. Effects of porcelain/alloy interfacial diffusion zones on thermo-mechanical strain.

J. Dent. Res. 63(5): 585; 1983.

Tung, T. S. Surface properties of hydroxyapatite in fluoride solution. Colliods and Surfaces 6: 283; 1983.

Tung, M. S.; Brown, W. E. Characterization and modification of electrochemical properties of teeth. J. Dent. Res. 62(1): 60; 1983. 
VanderHart; Atalla, R. H. Studies of microstructure in native celluloses using solid-state ${ }^{13} \mathrm{C}$ NMR. Preprints of the Intl. Dissolving and Specialty Pulps Conference, Boston, Ma 1983.

Vogel, G. L.; Chow, L. C.; Brown, W. E. A microanalytical procedure for the determination of calcium, phosphate and fluoride in eniamel biopsy samples. Caries Res. 17: 23; 1983.

Wagner, H. L.; Dillon, J. G. A low shear, high temperature rotational viscometer: the viscosity of ultrahigh molecular weight polyethylene, in Polymer Characterization, Adv. in Chem. Ser. 203, Craver, C. D., ed. ACS, Washington, DC 1983.

Wang, F. W. Applications of fluorescence techniques to polymer systems: polymer compatibility and segmental mobility. Polymer Preprints, Japan 32(1): 79 ; 1983.

Weiss, G. H.: Rubin, R. J. Random walks: theory and selected applications, Advances in Chem. Phys. 52: 363; 1983 (John Wiley \& Sons, Inc., NY).

Wu, W.; Cobb, E.; Dermann, K.; Rupp, N. W. A silver staining technique for detecting margin leakage of dental composite restorations: J. Biomed. Matl. Res., 17: 37; 1983.

Wu, W. Small angle neutron scattering of partially segregated polymer blends, Polymer 24: 43; 1983.

Wu, W; Cobb, W; Dermann, K. ; Rupp, N. W. Detecting margin leakage of dental composite restorations. J. Biomed. Mater. Res. 17: 37; 1983.

Bowen, R. L; Cobb, E. N. A method for bonding to dentin and ename1. JADA; in press.

Brauer, G. M.; Starisbury, J. A. Dental cements containing esters of syringic acid. J. Dent. Res. in press.

Broadhurst, M. G. Rigorous bounds for the calculated diectric constants of ferroelectric polymers. Ferroelectrics; in press.

Brown, W. E. ; Chow, L. C.; Mathew, M. Thermodynamics of hydroxyapatite surfaces. Croatica Chemica Acta; in press.

Brown, D. W. ; Lowry, R. E. ; Smith, L. E. Equilibrium acid concentrations in hydrolyzed polyester diols and polyester polyurethanes, Polymer preprints; in press.

Brown, D. W.; Lowry, R. E. ; Smith, L. E. Equilibrium acid concentrations in hydrolyzed polyesters and polyester polyurethane elastomers, $\mathrm{J}$. of Appl. Poly. Sci.; in press.

Chang, S. S.; Pummer, W. J.; Maurey, J. R. Fat-simulating and accelerating solvents for polyolefins and MWD of solvent extracts of polyethylenes.

Polymer; in press.

Chang, S. S. Migration of low molecular weight components from polymers. 1. methodology and diffusion of n-octadecane in polyolefins, Polymer; in press.

Chang, S. S. Specific heat of phenolic resins. ACS Polymer Preprints 24: in press.

Chang, S. S. Thermodynamic properties and glass transition of polystyrene. J. Polym. Sci.; in press. 
Dehl, R. E. Reply to "comments on 'on the characterization of porosity in PTFE-carbon composite implant materials by mercury porosimetry" "J. Biomed. Mater. Res.; in press.

DeReggi, A. S. Transduction phenomena in ferroelectric polymers and their role in pressure transducers. Proc. of the 1983 IEEE Int T. Symp. on Applications of Ferroelectrics, Gaithersburg, MD; in press.

DeReggi, A. S. Polymeric transduction phenomena and their role in biomedical applications; Inti. symp. on piezoelectricity in biomaterials and biomedical devices, Pisa, Italy; in press.

Dickens, B.; Marchal, J. Oxidation of polystyrene in solution. Polym.

Degrad. Stab.; in press.

Dickens, B. Thermal degradation study of polyisobutylene using factor-jump thermogravimetry. J. Thermal Anal.; in press.

Dickens, B. Factor-jump thermogravimetry as applied to the study of polymer degradation. Chapter in advances in fundamentals of polymer stability and degradation. Jellinek, H. H. G., ed. Elsevier, Publ.; in press.

Dickens, B.; Martin, J. W. ; Waksman, D. Solar type photolytic and thermal degradation of plates of poly(methyl methacrylate). Division of polymer chemistry preprints; ACS 24 ; in press.

Dickens, B.; Martin, J. W.; Waksman, D. Thermal and photolytic degradation of plates of poly(methyl methacrylate) containing monomer. Polymer; in press.

Fanconi, B. M. Fourier transform infrared spectroscopy of polymers-theory and application, J. of Test. and Evaluation; in press.

Fanconi, B. M. Chain scission and mechanical failure of polyethylene, J. of App 1. Phys.; in press.

Flynn, J. H.; Florin, R. Polymer degradation and pyrolysis mechanism, Chapter 4 in Pyrolysis and GC in Polymer Analysis, S. A. Liebman and E. J. Levy, eds. Marcel Dekker, Inc., New York; in press.

Furukawa, T.; Lovinger, A. J.; Davis, G. T.; Broadhurst, M. G. Dielectric hysteresis and nonlinearity in a $52 / 48$ mol percent copolymer of vinylidene fluoride and trifluoroethylene; in press.

Guttman, C. M. Some remarks in the history and development of the ASTM E-37 purity method. Purity Determinations by Thermal Methods, ASTM Symp. Series; in press.

Guttman, C. M.; DiMarzio, E. A. Monte carlo modeling of kinetics of polymer crystal growth-Regime III and its implications on chain morphology, J. Appl. Phys.; in press.

Hammonda, B.; Akacasu, A. Z.; Lodge, T. P.; Han, C. C. Dynamic scattering from bimodal polymer solutions. I. apparent diffusion coefficient. Macromolecules; in press.

Han, C. C. ; Amis, E. J.; Matsushita, Y. Dynamic light scattering measurements of polystyrene in semidilute theta solutions. Polymer; in press.

Hunston, D. L.; Bascom, W. S. Effects of lay-up, temperature, and loading rate in double cantilever beam tests of interlaminar crack growth. Composite Technology Review; in press. 
Hunston; D. L.; Wang, S. S.; Kinloch, A. J. Characterization of fracture behavior of adhesive joints. Intl. Symp. on .Adhesive Joints; in press.

Kinloch, A. J.; Shaw, s. J.; Tod, A. A. ; Hunston, D. L. Deformation and fracture of rubber toughened epoxy: 1. microstructure and fracture studies. Polymer; in press.

Kinloch, A. J.; Shaw, S. J.; Hunston, D. L. Deformation and fracture behavior of a rubber toughened epoxy: 2. failure criteria. Polymer; in press.

Lang, S. S.; DeReggi, A. S.; Mopsik, F. I.; Broadhurst, M. G. Pre-existing polarization and influence of electrode materials on PVF $_{2}$ electrets as determined by thermal pulse and pyroelectric methods. J. Appl. Phys.; in press.

Lovinger, A. J.; Furukawa, T.; Davis, G.T.; Broadhurst, M. G. Crystallographic changes characterizing the curie transition in three ferroelectric copolymers of vinylidene fluoride and trifluoroethylene. 1. as-crystallized samples; in press.

Lovinger, A. J.; Furukawa, T.; Davis. G. T.; Broadhurst, M. G. Crystallographic changes in characterizing the curie transition in three ferroelectiric copolymers of vinylidene fluoride and trifluoroethylene. 2. oriented or poled samples; in press.

Lodge, T. P.; Han, C. C; Akcasu, A. Z. Temperature dependence of dynamic light scattering in the intermediate momentum transfer region, Macromolecules; in press.

Ludeman, S. M. ; Shao, K-L; Zon, G.; Himes, V. L.; Mighel, A. D.; Takagi, S. 0 -iminyl esters of $\mathrm{N}, \mathrm{N}-\mathrm{bis}(2$-chloroethyl)phosphorodiamidic acid. synthesis, $x$-ray structure determination, and anticancer evaluation. J. Med. Chem.; in press.

McCrackin, F. L.; Guttman, C. M. ; Akcasu, A. Z. Monte carlo calculations of the hydrodynamic radii of polymers in theta and good solvents. Macromolecules; in press.

McKenna, G. B.; Zapas, L. J. Experiments on the small strain behavior of crosslinked natural rubber I. torsion, Polymer; in press.

McKenna, G. B.; Zapas, L. J. Experiments on the small strain behavior of . crosslinked natural rubber II. extension and compression. Polymer; in press.

Mopsik, F. I.; DeReggi, A. S. The poling behavior of polyvinylidene fluoride at room temperature. Appl. Phys. Lett.; in press.

Passaglia, E.; Khoury, F. A. Crystal growth kinetics and the lateral habits of polyethylene crystals; in press.

Peterlin, A.; DeCandia, F.; Perullo, A.; Vittoria, V. Transport properties of drawn isotactic polypropylene. Proc. Int1. Symp. on Polymers; in press.

Peterlin, A. Transport properties as an extremely sensitive indicator of the status of the amorphous component in the elastically and plastically deformed semicrystalline polymer. Proc. Int1. Symp. on Polymers; in press.

Phillips, J. C.; Peterlin, A. Diffusion of ethylacetate in strained polyethylene. J. Polym. Sci. (Polym. Sci. Ed.); in press.

Phillips, J. C.; Peterlin, A. ; Waters, P. F. Stress relaxation of polyvinylidene fluoride in ethyl acetate vapor, ACS Polymer Preprints; in press.

Phillips, J. C.; Peterlin, A.; Waters, P. F. The effect of elastic and plastic deformation on the solubility of ethyl acetate vapor in polyvinylidene fluoride $\left(\mathrm{PVF}_{2}\right)$, ACS Polymer Preprints; in press. 
Sanchez, I. C. On the nature of polymer interfaces and interphases, Polym. Eng. Sci.; in press.

Tung, M. S.; Brown, W. E. An intermediate state in hydrolysis of amorphous calcium phosphate. Calcif. Tiss. Interntl.; in press.

VanderHart, D. L.; Atalla, R. H. Native celluloses as composites of two distinct erystaliine forms, Science; in press.

Wang, F. W. ; Lowry, R. E.; Grant, W. H. In-situ monitoring of polymerization reactions by excimer fluorescence technique. Organic coatings and applied polymer science proceedings; in press.

Wang, F. W.; Lowry, R. E.; Grant, W. H. Novel excimer fluorescence method for monitoring polymerization. 1. Polymerization of methyl mathacrylate. Polymer; in press.

Waterstrat, R. M. The Hf-Os constitution diagram. J. of Less Common Metals; n press.

Wignall, $\tilde{u}$. D. ; Wu, W. A SANS investigation into the role of melting and recrystallization during solid state deformation of polyethylene, Polymer; in press.

Wu, W.; Fanconi. B. M. Post-curing of dental restorative resin, Polym. Eng. and Sci.; in press.

Wu, W.; Debelius, E. E.; Moffa, J. F.; Eilison, J. A. Subsurface damage layer of in-vivo worn dental composite restorations. J. Dent. Res.; in press.

Zapas, L. J.; Crissman, J. M. The necking of semicrystalline polymers in tension, Proc. of the workshop on instabilities and two phase systems, March 1983; in press.

Zapas, L. J.; Crissman, J. M. The creep and recovery behavior of ultra high molecular weight polyethylene in the region of small uniaxial deformation.

Polymer; in press.

\section{Special Reports}

Cassel, J. M. ; Tesk, J. A. ; Brauer, G. M. ; Antonueci, J. M.; Wu, W. ; McKinney, J. E. Properties and interactions of oral structures and restorative materials. Annual report to National Institute of Dental Research (NIDR); October 1982 to September 1983; in press.

Books

Tesk, J. A. Solders and fluxes. Chapter in Dentists Desk Reference, Materials, Instruments and Equipment, 2nd ed., American Dental

Association Publications; in press.

Tesk, J. A.; Waterstrat, R. M. Physical metallurgy of dental casting alloys, Sections, $C, D, E$, and F. Encyclopedia of Materials Science and Engineering, Pergamon Press; in press.

\section{Consulting and Advisory Services}

NIDR Grant Proposal Reviewer

J. A. Tesk

American National Standards Institute

B. Dickens 


\section{External Recognition}

Dr. G. C. Paffenbarger

Research Associate, American Dental Association

Distinguished Service Award of American Dental Association

November 1982

Dr. G. C. Paffenbarger

Research Associate, American Dental Association

Guest of Honor at 39 th Annual Dental Post-College Assembly

Dental Faculty and Dental Alumni Assoc., Ohio State University

April 1983

New SRMS

SRM 1470, Polyester Film SRM for Gas Transmission Measurements

J. D. Barnes, 1983

\section{Patents}

Cementitious Dental Compositions Which do not Inhibit Polymerization

G. M. Brauer, H. Argentar, and J. W. Stansbury

U. S. Patent \#4,3E2,510

December 7, 1982

Method for Obtaining Strong Adhesive Bonding of Composites to Dentin, Ename 1 and other Substrates

R. L. Bowen

Filed January 10, 1983

Isotonic Monomer Formulations

R. L. Bowen

U. S. Patent $\sharp 4,373,035$

February 8,1983

Compositions and Methods for Topically Fluoridating or

Mineralizing Cental Tissue

W. E. Brown and L. C. Chow

Filed March 25, 1983

\section{Industrial Interactions}

\section{Archival Technical Papers Coauthored with Industrial Employees}

Bascom, W. D.; Hunston, D. L. The fracture of epoxy and elastomer-modified epoxy polymers. Chapter 14 in Adhesion 6. Allen, K. W., ed. London, Applied Science Publishers, 1982.

Crissman, J. M. Participated in the 4th Annual Plastic Piping Materials Workshop sponsored by the Gas Research Inst., Chicago, IL April 1983.

VanderHart, D. L; Atallas, R. H. Studies of microstructure in native celluloses using solid-state ${ }^{13} \mathrm{C} \mathrm{NMR}$, Preprints of the Intl. Dissolving and Speciaity Pulps Conf., Boston, 1983.

Hunston, D. L. ; Bascom, W. D. Effects of lay-up, temperature, and loading rate in double cantilever beam tests of interlaminar crack growth, Composite Technology Review; in press.

VanderHart, D. L. ; Atalla, R. H. Native celluloses as composites of two distinct crystalline forms, Science; in press. 
Rohm and Haas, Inc. $/ 6 / 1 / 83 / 0$ iscussed the research associate program and expressed interest in collaborating with NBS in further work on the photooxidation and thermal degradation of poly (methylmethacrylate).

Feldmuhle Co./5/10/83/Bonding test for forced-fused-tometal systems.

Shofu Dental Manufacturing/4/29/83/Testing amalgam, bonding of composites to dentin, vanallate cements.

Nipon Silken Dental Co./11/24/82/Casting of dental alloys, effects of temperature and investments.

G. C. Dental Co./10/18/82/Clinical behavior of glass-ionomer cements and new dental cements.

Bayer AG/3/22/83/Composite chemistry including acceleration, monomer and bonding to fillers.

ICI Pharmaceuticals/3/15/83/Composite resins, strength, wear, filler loading, coupling agents.

Dentsply International/4/26/83/Pursue agreement for industrial research associate.

Coe Laboratories/4/19/83/Dental composite and surgical bone cement chemistry.

Howmedica Inc./2/24/83/Modification leading to improvement of bone cement-implant system.

Colgate Palmolive Co./2/25/83/Fluoridation techniques in development and fluoride-calcium phosphate chemistry.

Health Services Intl., Inc./1/22/83/Information on dental products for third world nations.

Bell Laboratories/1/20/83/Overview of Programs.

Sulzur Brothers/10/25/82/Discuss merits of bone cement implant system as cementless orthopedic implants.

Martin Marietta/1/19/1983/Overview of Programs.

American Dental Association/2/28/83/0verview of Programs.

Gas Research Institute/5/24/83/Overview of Programs. 

NBS.114A (REV. 2-8C)

U.S. DEPT. OF COMM.

BIBLIOGRAPHIC DATA

SHEET (See in structions)

1. PUBLICATION OR REPORT NO.

NBSIR $84-2813$

2. Performing Organ. Report No.

3. Publication Date

January 1984

4. TITLE AND SUBTITLE

Polymer Science and Standards Division Annual Report 1983

5. AUTHOR(S)

L. E. Smith, M. G. Broadhurst, G. T. Davis, F. W. Wang, B. M. Fanconi, J. A. Tes

6. PERFORMING ORGANIZATION (If joint or other than NBS, see in struction s)

NATIONAL BUREAU OF STANDARDS

DEPARTMENT OF COMMERCE

WASHINGTON, D.C. 20234

9. SPONSORING ORGANIZATIEN NAIME AND COMPLETE ADDRESS (Street. City. Stote, ZIP)

NBS

Washington, DC 20234

10. SUPPLEMENTARY NOTES

Document describes a computer program; SF-185, FIPS Software Summary, is attached.

11. ABSTRACT (A 200-word or less factual summary of most significant information. If document includes a significant bibliography or literature survey, mention it here)

Although synthetic polymers have been used as materials for technology during oniy three quarters of a century, they have left little of our economy, technology, industry, science, and culture untouched. We have moved rapidly into an age in which an evergrowing number of humanity's needs are served by polymers. The volume currentTy produced exceeds that of steel and forms the basis of industries which add over $\$ 106$ billion of value by manufacturer (a measure of the relative economic importance of manufacturing among industries) and provides 3.3. million jobs. Recent summaries show that polymers and polymer composites research already accounts for about 47 percent. of the total industrial R\&D expenditure for metals, polymers, and inorganic materials. Among these materials, polymers also constitute about 40 percent of the yalue added by manufacturer, 45 percent of the jobs, 49 percent of the number of scientific publications, and 42 percent of the American Society for Testing and Materials (ASTM) standards.

12. KEYY WORDS (Six to twelve entries: alphabetical order: capitalize only proper names; and separate kay words by semicolons) Chemical durability; characterization; dental and medical materials; dielectric plastics; performance; polymers; mechanical durability; migration.

13. AVAILABILITY

X Unlimited

For Official Distribution. Do Not Release to NTIS Order From Superintendent of Documents, U.S. Government Printing Office. Washington, D.C.
20402 .

X Order From National Technical Information Service (NTIS), Springfield, VA. 2216I

14. NO. OF

PRINTED PAGES

110

15. Price

$\$ 13.00$ 


